\title{
Elucidation of the early steps of ergot alkaloid synthesis by pathway reconstruction in Aspergillus nidulans
}

Katy Ryan

Follow this and additional works at: https://researchrepository.wvu.edu/etd

\section{Recommended Citation}

Ryan, Katy, "Elucidation of the early steps of ergot alkaloid synthesis by pathway reconstruction in Aspergillus nidulans" (2014). Graduate Theses, Dissertations, and Problem Reports. 6545.

https://researchrepository.wvu.edu/etd/6545

This Dissertation is protected by copyright and/or related rights. It has been brought to you by the The Research Repository @ WVU with permission from the rights-holder(s). You are free to use this Dissertation in any way that is permitted by the copyright and related rights legislation that applies to your use. For other uses you must obtain permission from the rights-holder(s) directly, unless additional rights are indicated by a Creative Commons license in the record and/ or on the work itself. This Dissertation has been accepted for inclusion in WVU Graduate Theses, Dissertations, and Problem Reports collection by an authorized administrator of The Research Repository @ WVU.

For more information, please contact researchrepository@mail.wvu.edu. 


\title{
Elucidation of the early steps of ergot alkaloid synthesis by pathway reconstruction in Aspergillus nidulans
}

\author{
Katy Ryan \\ Dissertation submitted to the \\ Davis College of Agriculture, Natural Resources and Design \\ at West Virginia University \\ in partial fulfillment of the requirements for the degree of \\ Doctor of Philosophy in \\ Genetics and Developmental Biology \\ Daniel Panaccione, Ph.D., Chair \\ Vagner Benedito, Ph.D. \\ Kenneth Blemings, Ph.D. \\ William MacDonald, Ph.D. \\ Jianbo Yao, Ph.D. \\ Division of Plant and Soil Sciences
}

Morgantown, West Virginia

2014

Keywords: Ergot alkaloids, mycotoxins, fungi, genetic engineering, Aspergillus

Copyright 2014 Katy Ryan 


\section{ABSTRACT \\ Elucidation of the early steps of ergot alkaloid synthesis by \\ pathway reconstruction in Aspergillus nidulans}

\section{Katy Ryan}

Ergot alkaloids are pharmaceutically and agriculturally important secondary metabolites produced by several species of fungi in the Clavicipitaceae (order Hypocreales) and Trichocomaceae (order Eurotiales). Ergot alkaloid pathways vary among producers due to genetic variation within ergot alkaloid synthesizing (eas) gene clusters. However, the early steps of the pathway leading to the formation of chanoclavine-I appear to be evolutionarily conserved among producers. Chanoclavine-I synthesis occurs in at least five steps, and knockout and heterologous expression studies have demonstrated that four genes, $d m a W$, easF, easE, and eas $C$, are required for pathway steps prior to chanoclavine-I formation. It was unknown whether these genes were sufficient to encode synthesis of chanoclavine-I or if another gene not located within the eas cluster was involved. DmaW performs the determinant step of alkaloid synthesis by prenylating tryptophan to produce dimethylallyltryptophan (DMAT). The easF gene encodes a methyltransferase responsible for the conversion of DMAT to N-MeDMAT. The specific roles of EasE and EasC have not been established, though bioinformatics suggests that easE encodes an oxidoreductase and EasC is a catalase. The proposed biochemical scheme for chanoclavine-I synthesis involves two oxidation reactions for conversion of N-Me-DMAT to chanoclavine-I; thus, involvement of additional genes is plausible since easE encodes the only oxidase among the genes listed. Four genes, dmaW, easF, easE, and eas $C$ were amplified from the human pathogen Aspergillus fumigatus and transformed into Aspergillus nidulans, which does not contain any of the ergot alkaloid synthesis genes and produces no ergot alkaloids. HPLC and LC/MS analyses demonstrated that $d m a W$, easF, easE, and eas $C$ were sufficient for chanoclavine-I synthesis in $A$. nidulans; however, the possibility that uncharacterized activities already present in $A$. nidulans contributed to chanoclavine-I synthesis cannot be excluded. Combinations of the four genes listed above were amplified and transformed in A. nidulans to gain insights into the roles of EasE and EasC. Aspergillus nidulans transformant WFC (containing the genes $d m a W$, eas $F$, and eas $C$ ) accumulated a novel ergot alkaloid not detected in control strains or $A$. nidulans WFE (containing the genes dmaW, eas $F$, and easE). The structure of the newly discovered compound was elucidated by labeling studies, high-resolution LC/MS, and NMR and given the trivial name ergotryptamine. Ergotryptamine feeding studies conducted with $A$. nidulans WFE and eas $C$ knockout $A$. fumigatus strains revealed that this metabolite is not an intermediate in chanoclavine-I synthesis. Ergotryptamine also was detected in several species of Epichloë, and further investigations established that ergotryptamine is the same molecule provisionally characterized as 6,7-secolysergine in previous work with Epichloë species. I conclude that the four genes listed above are sufficient for the synthesis of chanoclavine-I in $A$. nidulans and reveal the chemical structure of a novel metabolite produced during the early steps of ergot alkaloid synthesis. The approach of expressing ergot alkaloid pathway genes in A. nidulans provides a mechanism for better understanding of the early steps in ergot alkaloid synthesis. 


\section{Acknowledgements}

First off, I'd like to thank the most important person in my life my man Matt Smoot. He knows why. Secondly, I would like to thank my family for their total support and guidance throughout my time in school. I'm very grateful for the guidance and support from my advisor, Dan Panaccione, and committee members who helped me get to where I am today, and helped me grow as a student and scientist. I'd like to thank Novruz Akhmedov for his help with chemical analyses. The members of the lab that I worked in also helped shape my time as a student and contributed to the development of this research, Prashanthi Mulinti, Chris Moore, Julia Bilovol, Chris Powell, Sarah Robinson, Katrina Schlum and Tanya Dilan. And finally, I would like to extend thanks to my fellow graduate students, who helped me in my work as well as offered encouragement and friendship along the way. 


\section{Table of Contents}

Abstract

Acknowledgments

Table of Contents

ii

iii

iv

Chapter 1: Review of Literature

$\begin{array}{ll}\text { Overview } & 2 \\ \text { Ergot alkaloids in agriculture } & 2 \\ \text { Ergot alkaloids in medicine } & 3 \\ \text { Ergot alkaloid producers } & 6 \\ \text { Ecological roles } & 8 \\ \text { Ergot alkaloid profiles } & 9 \\ \text { Pathway regulation } & 10 \\ \text { Gene cluster variation } & 10 \\ \text { Early steps of the pathway } & 11 \\ \text { Late steps of the pathway } & 12 \\ \text { Project objectives } & 12 \\ \text { References } & 13 \\ \text { Figures } & 20\end{array}$

Chapter 2: Partial Reconstruction of the Ergot Alkaloid Pathway by Heterologous Gene Expression in Aspergillus nidulans

$\begin{array}{ll}\text { Abstract } & 23 \\ \text { Introduction } & 23 \\ \text { Methods } & 24 \\ \text { Results } & 27 \\ \text { Discussion } & 28 \\ \text { References } & 30 \\ \text { Figures } & 32\end{array}$

Chapter 3: Identification and structural elucidation of a new ergot alkaloid, ergotryptamine, produced by genetically modified Aspergillus nidulans and natural variants of Epichloë

$\begin{array}{ll}\text { Abstract } & 38 \\ \text { Introduction } & 38 \\ \text { Methods } & 39 \\ \text { Results and Discussion } & 43 \\ \text { References } & 50 \\ \text { Tables and Figures } & 52\end{array}$

Chapter 4: Heterologous expression of ergot alkaloid synthesizing genes in Aspergillus nidulans, additional mutants

$\begin{array}{ll}\text { Introduction } & 63 \\ \text { Methods } & 63 \\ \text { Results and Discussion } & 64 \\ \text { References } & 65 \\ \text { Figures } & 66\end{array}$

Chapter 5: Investigating the involvement of eas $A$ in lysergol and cycloclavine synthesis

$\begin{array}{ll}\text { Introduction } & 70 \\ \text { Methods } & 70 \\ \text { Results and Discussion } & 72 \\ \text { References } & 74 \\ \text { Figures } & 76\end{array}$

Chapter 6: Modification of grass endophytes for altered ergot alkaloid production

$\begin{array}{ll}\text { Introduction } & 80 \\ \text { Methods } & 80 \\ \text { Results and Discussion } & 83 \\ \text { References } & 84 \\ \text { Figures } & 86\end{array}$

Summary $\quad 88$

Curriculum Vitae $\quad 89$ 


\section{Chapter 1}

\section{Review of Literature}




\section{Overview}

Ergot alkaloids are a diverse family of indole-derived mycotoxins. Several species of fungi produce these secondary metabolites which are both pharmaceutically and agriculturally important (reviewed in Panaccione, 2010). The biological activities of ergot alkaloids are due to their affinity for neurotransmitter receptors which leads to a variety of effects in humans and animals. Apart from neurological effects, ergot alkaloids have also been shown to possess different properties such as antibiotic, insecticidal, and nematicidal activity. Throughout the past decade, there have been numerous studies conducted to better understand the ergot alkaloid pathway and manipulate it for applicable purposes. While many such studies have proved fruitful and efficacious, there are many questions involving the biosynthesis of different alkaloids that remain unresolved. The continual advancement of ergot alkaloid studies furthers the possibilities of understanding their true value for the future of pharmaceuticals, as well as, the regulation of alkaloids in agricultural settings.

\section{Ergot Alkaloids in Agriculture}

Human poisoning by ergot alkaloids dates back centuries and is mainly due to consumption of rye-based foods that are contaminated by Claviceps purpurea, a fungal pathogen of rye and other grasses. C. purpurea infects flowers of grasses and replaces developing seeds with a hard, dark overwintering structure known as a sclerotium (Belser-Ehrlich et al., 2013; Tudzynski and Scheffer, 2004). Mature sclerotia harbor newly-formed ascospores and accumulate high concentrations of ergot alkaloids, though concentrations will vary depending on fungal strains involved and environmental factors. Spores can be transferred to other developing seeds within the plant or to neighboring vegetation by herbivores or environmental vectors, furthering the spread of infection. If sclerotia are not removed before processing of the rye, ergot alkaloid contamination may occur in foods and feed.

As relatively stable molecules, ergot alkaloids that can withstand high temperatures of baking ovens with little degradation and tolerate harsh $\mathrm{pH}$ conditions in the human digestive system, which makes them successful toxins (Merkel et al., 2012). Ergot alkaloid toxicosis in humans may result in a variety of symptoms including hallucinations, involuntary muscle contractions, miscarriages, and vasoconstriction which, in severe cases, may cause gangrene (Eadie, 2003). Though these mycotoxins have caused severe epidemics in medieval history, human poisoning due to ingestion of ergot alkaloids is now rare in developed countries due to improved methods of cleaning grain and broadening diets. There have been several reported outbreaks of human toxicosis within the last century in countries such as Russia, England, France, India, and Ethiopia (Belser-Ehrlich et al., 2013). The latest reported outbreak of human toxicosis was found in Ethiopia in 2001. Though the total number of victims was not reported, 
18 patients were diagnosed with ergot toxicosis and three deaths resulted from the outbreak (Urga et al., 2002). Many developed countries now have federal guidelines limiting the amount of ergot in food and feed. In the United States, the maximum net weight limit of ergot in rye is $0.3 \%$, while barley and oats have a lower maximum net weight limit of $0.1 \%$ (7 CFR 810).

Contaminated rye in feed can cause many of the same symptoms in livestock as it does in humans. Epichloë species (which are close relatives of Claviceps spp.) are mutualistic symbionts of grasses and capable of producing ergot alkaloids, particularly high concentrations of the potent toxin ergovaline (Clay and Schardl, 2002). Consumption of alkaloid-contaminated grasses, as well as sclerotia infected feed, by livestock has been shown to cause weight loss, low fertility, low milk production, lethargy, and elevated body temperature (Schardl and Phillips, 1997). Severe ergot outbreaks can cause economic loss to agricultural industries. One of the latest reported outbreaks was recorded in Brazil in 1999 where 97 cows (52\% of cattle from three farms) showed symptoms of ergot toxicosis (Ilha et al., 2003). These cattle were reported to have hyperthermia, lethargy, and a decrease in milk yield that at times reached a $100 \%$ decrease. Livestock outbreaks are much more common than human epidemics and occur sporadically across the world because animal feed regulations are not as strictly regulated.

Though ergot alkaloid contamination data is not available for the US, the European commission recently conducted a study on ergot alkaloid contamination in foods and feed from 14 European countries (European Food Safety Authority, 2012). A total of 25,558 samples were collected between years 2004-2011 and tested for the presence of several potent ergot alkaloids: ergonovine, ergotamine, ergosine, ergocristine, $\alpha$ - and $\beta$-ergocryptine, and ergocornine. A total of 2,279 samples tested positive for alkaloids, and not surprisingly, rye samples contained the highest concentrations of ergot alkaloids. The positive samples were divided into 3 categories with 1716 contaminated samples classified as food sources, 496 samples were categorized as feed, and 67 samples were unprocessed material. Despite the high number of positive samples, the EFSA declared that ergot alkaloid contamination in food and feed is not a concern in European countries due to the low concentrations detected. The European commission also released data from the EFSA's comprehensive European food consumption database stating that chronic exposure to ergot alkaloids in adults is about 0.007-0.08 $\mu \mathrm{g} / \mathrm{kg} / \mathrm{day}$. Based off of food eating habit surveys, they estimated that toddlers are the age group receiving the highest concentration of ergot alkaloids a day.

\section{Ergot Alkaloids in medicine}

Ergot alkaloids are similar in structure to several neurotransmitters which explains their affinity for neurotransmitter receptors like those for dopamine, serotonin, adrenaline, and noradrenaline (Pertz 
and Eich, 1999; Pertz, 1996). Researchers have taken advantage of the biological activities of ergot alkaloids in order to produce pharmaceutical treatments for various ailments; thus showing the beneficial nature of ergot alkaloids in the medical field. Even during the Middle Ages ergot alkaloids were used as therapeutic agents (Tudzynski et al., 2001). Midwives have utilized these compounds to aid in childbirth or to induce uterine contractions for abortion; though at the time this practice was hazardous as there was no way to properly measure the amount of ergot alkaloids administered.

Several ergot alkaloids, whether natural or semisynthetic, are commercially available. Ergotamine and dihydroergotamine are commonly used to treat migraines because of their affinity for 5-hydroxytryptamine $(5-\mathrm{Ht})$ receptors; this interaction leads to vasoconstriction (Tfelt-Hansen and Koehler, 2008). Methylergonovine was used in the US to control bleeding during and after childbirth (Howard et al., 1964). Semisynthetic ergot alkaloids cabergoline and pergolide are both used to treat Parkinson's disease in other countries because of their affinity for dopamine receptors. Pergolide was formerly used for this purpose in the US; however, it was taken off the market by the FDA due to its severe side effects which lead to valvular heart disease (United States Food and Drug Administration, 2007; Zanettini et al., 2007). Lysergol is currently being used in the development of antibiotics because it increases the antibiotic activity of various drugs such as the conventional compound nalidixic acid, which many strains of bacteria have become resistant against (Maurya et al., 2013; Khanuja, 2006). As a bioenhancer, lysergol allows for a lower concentration of antibiotics to be administered, thereby decreasing the frequency of microbial resistance. Another pharmaceutically significant alkaloid is ergocrytine which inhibits the release of prolactin, and therefore is used to treat prolactin-related disorders (Schneider et al., 1977).

Several ergot alkaloids possess desirable properties making them promising candidates for medical application. Agroclavine and festuclavine were shown to have cytostatic properties when incubated with mouse lymphoma cultures (Eich et al., 1984). It has been proposed that these compounds inhibit DNA synthesis which makes them promising for cancer research. The simple clavine alkaloid chanoclavine, and several analogues, were shown to stimulate dopamine receptors in mice (Wantanbe et al., 1987). Fumigaclavine $C$ has been shown to act as an anti-inflammatory agent by inhibiting the expression of several proinflammatory cytokines, and has also been shown to specifically reduce liver damage and prevent colitis in mice (Wu et al., 2005; Zhao et al., 2004). However, ergot alkaloid utility in medicine is limited due to severe side effects caused by binding to multiple neurotransmitter receptors. For clinical applications ergot alkaloids may need to be chemically modified to alter receptor specificities. 
Large scale production of ergot alkaloids can be an obstacle for pharmaceutical use (reviewed in Tudzynski et al., 2001). Some fungi only generate ergot alkaloids when infecting a plant host which limits industrial productivity, and furthermore isolation and purification techniques may be expensive. Claviceps species are the main ergot alkaloid producing fungi used for biotechnological applications because they produce moderate concentrations of pharmaceutically popular alkaloids like ergopeptines and lysergic acid amides. Large scale ergot production began in the 1930s and involved inoculating Claviceps spores on fields of rye grasses and later collecting developed sclerotia for alkaloid extraction. This technique is still applied today; however it is not ideal because ergot alkaloid production is too dependent on environmental conditions and land availability is restricted for the protection of other grasses. Advancements in sclerotia field cultivation have led to the development of a hybrid wheat strain which possesses induced male-sterility causing florets to remain open for an extended period allowing for a higher chance of infection by Claviceps spp. (Nemeth et al., 1999). Apart from field cultivation, industries also rely on saprophytic cultures for alkaloid production, though fewer strains are available for this technique. Claviceps spp. are grown in submerged cultures containing a high sugar medium which induces a sclerotia-like state for ergot alkaloid production. Alkaloids can be easily extracted from liquid media and purified for commercial availability. A draw back to this method is that recultured strains are not genetically stable and quickly degenerate producing lower concentrations of alkaloids over time. In 2010 approximately $20,000 \mathrm{~kg}$ of ergot alkaloids were produced for pharmaceutical means, approximately $10,000 \mathrm{~kg}$ from each alkaloid extraction technique (Hulvova et al., 2013).

This review would not be complete without mention of the most infamous ergot alkaloid derivative lysergic acid diethylamide (LSD). This synthetic compound was discovered by Albert Hofmann for psychotherapeutic treatments and was found to be a very potent hallucinogen altering the state of consciousness in the microgram level (Passie et al., 2008). LSD is now a schedule I controlled substance in the US (21 USC Ch. 13). Natural compounds similar to LSD are ergine (lysergic acid amide) and lysergic acid $\alpha$-hydroxyethylamide (LAH). Ergine has been studied extensively and found to have powerful hallucinogenic effects, however it is not as potent as LSD and is classified as a schedule III controlled substance (Halpern, 2004; 21 USC Ch. 13). In ancient Central America, Aztec priests would ingest morning glory seeds, which normally contain high concentrations of ergine and other ergot alkaloids, as part of a sacred tradition allowing them to "communicate" with the gods (Schultes and Hofmann, 1992). Literature on the biological activities of LAH is lacking, though there is evidence that this alkaloid causes 
bizarre effects on animals such as excitability, pupil dilation, and changes in heart rate and respiration (Glasser, 1961). Based on these findings, it seems likely that LAH is a psychedelic molecule.

\section{Ergot Alkaloid Producers}

\section{Aspergillus species}

Aspergillus fumigatus is a common saprophyte and opportunistic human pathogen causing aspergillosis in immunosuppressed individuals (Latgé, 1999). It is classified in the phylum Ascomycota family Trichocomaceae and was originally thought to be imperfect, lacking the mechanisms to reproduce sexually, until O'Gorman et al. (2009) reported the sexual state. A. fumigatus has a high tolerance to varying environmental conditions and is important in agricultural settings because it can spoil silage and hay (Scudamore and Livesey, 1997).

A. fumigatus synthesizes simple clavine alkaloids rather than pharmaceutically popular lysergic acid-derived alkaloids. Most variants accumulate festuclavine, fumigaclavine $A$, fumigaclavine $B$, and fumigaclavine $C$; though there are natural strains with mutations that end progress of the pathway before the ultimate end product fumigaclavine C (Robinson and Panaccione, 2012). Other Aspergillus spp. have been reported to produce ergot alkaloids such as Aspergillus japonicus and Aspergillus tamarii (Furuta et al., 1982; Janardhanan et al., 1984). Recently, a new alkaloid was discovered in A. fumigatus and added to the clavine family named fumigaclavine D (Cano et al., 2013); this compound has yet to be reported in other fungi, and genetic and enzymatic bases for its synthesis remain unknown.

In A. fumigatus ergot alkaloid production is linked to asexual reproduction. The bristle $\mathrm{A}$ gene is a transcription factor regulating formation of asexual structures like conidiophores and conidia. In brlA knockout $A$. fumigatus cultures, conidiophores failed to develop properly and ergot alkaloids were not detected in fungal extracts (Coyle et al., 2007). Asexual capabilities and ergot alkaloid production were restored with brlA complementation. Alkaloid localization is not fully understood; however, conidia are known to harbor an abundance of these metabolites (Panaccione and Coyle, 2005). Mulinti et al. (2014) found that pathway end products accumulate to relatively high levels in conidia while pathway intermediates are detected in higher concentrations in entire conidiating cultures providing evidence for differential localization patterns. Attempts have been made to determine if ergot alkaloids are localized on the outside surface of fungal cell walls or contained internally without success; either way alkaloids can be easily extracted from sporulating cultures by incubation with methanol (Panaccione and Coyle, 2005)

A. fumigatus is a model organism for studying ergot alkaloid production because it produces alkaloids in a saprophytic state and exhibits a high growth rate. Several genes in $A$. fumigatus have been 
investigated through knockout and complementation studies, as well as heterologous expression in E.coli, such as dmaW, easF, easC, easE, easD, easA, easL, and easM (Coyle and Panaccione, 2005; Goetz et al., 2011; Unsöld and Li, 2005; Rigbers and Li, 2008; Coyle et al., 2010; Unsöld and Li, 2006; Wallwey et al., 2010a; Wallwey et al., 2010 b; Bilovol and Panaccione, not published). Many of the knockout strains previously generated were found to be invaluable because they served as recipient strains for the study of ergot alkaloid synthesizing genes specific to other producers (Coyle et al., 2010; Robinson and Panaccione, 2014; Bilovol and Panaccione, not published). Recent advancements in pathway manipulation have led to the development of mutant $A$. fumigatus strains capable of producing the pharmaceutically appealing compound lysergic acid (Robinson and Panaccione, 2014).

\section{Epichloë species}

Epichloë spp. are close relatives of Claviceps spp. and classified in the Clavicipitaceae family. They can act as either plant parasites (when in the sexual state) or mutualistic symbionts (when in the asexual state) of Poöideae grasses. Epichloë anamorphs are commonly used in alkaloid studies and were previously referred to as Neotyphodium spp.; however due to recent changes in nomenclature rulings, Neotyphodium spp. have been realigned with Epichloë (Leuchtmann et al., 2014).

Tall fescue (Festuca arundinacea) and perennial ryegrass (Lolium perenne) are two well-studied grasses for Epichloë/plant interactions. Mycelia of Epichloë grow throughout the above-ground tissues of grasses and into developing seeds allowing for transmission (Clay and Schardl, 2002). Fungal growth has been shown to be synchronized with plant growth and ceases when leaf growth stops making these fungi ideal mutualists (Christensen et al., 2002). However, telomorphic Epichloë strains develop ectophytic sexual reproductive structures around the host inflorescence which prevents plant reproduction and damages host tissue (Schardl et al., 2004). Anamorphic Epichloë strains lack the mechanisms to reproduce sexually and therefore do not have a parasitic stage.

The Epichloë /plant symbiosis offers many benefits to the plant such as drought tolerance, salt tolerance, and disease resistance (Clay and Schardl, 2002). Ergot alkaloid producing endophytes are also advantageous because they repel herbivores. Epichloë spp. generate an array of ergot alkaloids similar to that of $C$. purpurea, producing lysergic acid amides and ergopeptines. However, ergot alkaloid synthesizing genes are difficult to study in Epichloë spp. because they exhibit a slow growth rate and most strains only produce alkaloids when infecting grasses. On the other hand, this endophyte can be easily cultured in laboratory settings and reinfected into host tissue. Two ergot genes have been investigated in Epichloë sp. isolate Lp1 by knockout and complementation studies, dmaW and IpsA (Wang et al., 2004; Panaccione et al., 2001). Epichloë sp. isolate Lp1 knockout strains were not only 
important for understanding alkaloid biosynthesis but also for gaining insight into the biological roles of these compounds (see subsection "Ecological roles of ergot alkaloid").

Turf grasses may be improved with further research on ergot alkaloid producing endophytes due to the fact that some alkaloids possess insecticidal and/or nematocidal properties. However, ergot alkaloid producing Epichloë spp. are a concern in foraging grasses. As previously discussed, cattle can exhibit harmful effects from ingestion of alkaloids. It may be possible to utilize alkaloid-producing endophytes to improve plant health in foraging grasses; however, these grasses need to be tested extensively to ensure no detrimental effects on livestock. Other researchers would argue that the best way to improve foraging grasses is to utilize endophytes that do not produce ergot alkaloids (Parish et al., 2003; Bouton et al., 2002).

Morning glory fungi

It was recently discovered that morning glories harbor symbiotic, ergot alkaloid-producing fungi (Steiner et al., 2006). This newly discovered genus was given the name Periglandula due to fungus's association with secretory glands on the leaf (Steiner et al., 2011). As of now, two separate species were identified and sequenced: Periglandula ipomoeae which infects Ipomoea asarifolia and Periglandula turbinae which infects Turbina corymbosa. Phylogenetic trees place these fungi in the Clavicipitaceae which contains other well-known ergot alkaloid producers. There are at least 23 Ipomoea species with ergot alkaloid producing endophytes (Eich, 2008). Fungal mycelia may be visible on the plant leaf surface as dense white fuzz seen on Ipomoea hildebrandtii; however, in some plants like Ipomoea tricolor the fungus is not visible and only grows internally (Ahimsa-Muller et al., 2007). Transmission occurs by fungal growth into developing seeds. The alkaloids produced by morning glory fungi are similar to those of other clavicipitaceous fungi, synthesizing amides of lysergic acid and ergopeptines (Eich, 2008). Two morning glories associate with endophytes that produce unique alkaloids, lysergol and cycloclavine; however the biosynthetic origin of the compounds is unknown. Though interest in these fungi has increased, they have proven to be particularly difficult to study due to the fact that they are unable to be effectively cultured and cannot be transferred to a new host (Steiner et al., 2011). On the other hand, endophytes can be easily eliminated from plants by treating seeds with fungicides (Kucht et al., 2004).

\section{Ecological Roles of Ergot Alkaloids}

There is evidence that ergot alkaloids serve a role in fungal host preservation as herbivore feeding deterrents. Panaccione et al. (2006a) showed that rabbits preferred to feed on ergot alkaloidfree endophytic grasses (dmaW ko Epichloë sp. isolate Lp1) rather than grasses that accumulated ergot 
alkaloids (wt Epichloë sp. isolate Lp1). In another study, grasses infected with non-ergot alkaloid producing endophytes were more susceptible to the grass-feeding caterpillar, Agrotis ipsilon, than grasses containing alkaloids (Potter et al., 2008). Timper et al. (2005) found that ergot alkaloidproducing strains of Epichloë coenophiala repelled nematodes Pratylenchus zeae and Pratylenchus scribneri from tall fescue (Festuca arundinacea). Ergovaline and $\alpha$-ergocryptine were discovered to be lethal to $P$. scribneri at low concentrations of 5 and $50 \mu \mathrm{g} / \mathrm{ml}$, respectively (Bacetty et al., 2009). However, other studies involving different plant/fungal/nematode interactions have found that ergot alkaloids do not play a role in suppression of nematode activity (Panaccione et al., 2006b; Ball et al., 1997). Studies involving the ecological impacts of ergot alkaloids are limited to grass endophytes. Though literature is lacking for the benefits of ergot alkaloids to plant parasites and saprophytes, it seems probable that these fungi utilize ergot alkaloids as defensive tools to increase their chances of survival.

\section{Ergot Alkaloid Profiles}

The total yield of ergot alkaloids from a single producer does not only consist of pathway end products but also a buildup of intermediates, as well as alternate intermediates from branches in the pathway (Panaccione 2005). For example, some Periglandula spp. accumulate high concentrations of ergopeptines, as well as pathway branch products ergonovine and LAH (Beaulieu et al., 2013). A. fumigatus extracts from mature cultures may contain high concentrations of intermediate festuclavine and end product fumigaclavine $\mathrm{C}$, while other intermediates may not be detected or accumulate to low concentrations (Panaccione and Coyle, 2005). C. purpurea extracts may contain varying concentrations of several end products and branch products such as ergocryptine, ergotamine, ergonovine, ergocornine, ergosine, and ergocristine (Amici et al., 1969; Vining and Taber, 1959). Early pathway intermediates are usually not detected in fungal extracts (with the exception of genetically modified strains and natural mutant isolates where the pathway ends prematurely) suggesting a high level of efficiency in the early pathway steps.

Ergot alkaloid profiles may also contain shunt and spur products. For example, setoclavine and isosetoclavine are unique ergot alkaloids formed via oxidation of agroclavine by peroxidases, and found to accumulate in cultures with a buildup of agroclavine including mutant Epichloë and Aspergillus strains (Panaccione et al., 2003; Coyle et al., 2010). Clavicipitic acid is an unusual alkaloid which is hypothesized to be formed by an oxidoreductase (EasE) acting out of order to prematurely close the third ergoline ring. Ergine accumulates in many producers in the Clavicipitaceae and is proposed to be a stable product from ergopeptine catabolism (Panaccione et al., 2003). Therefore, ergot alkaloid profiles are 
diverse among organisms and complex within an organism. A buildup of intermediates with varying biological properties may be advantageous for survival of the fungus and/or host plant.

\section{Pathway regulation}

Several factors are known to influence the ergot alkaloid pathway. High inorganic phosphate concentrations hinder alkaloid production in Claviceps purpurea by decreasing the expression of ergot alkaloid synthesizing (eas) genes (Haarmann et al., 2005; Robbers et al., 1972; Rao et al., 1977). Ergot alkaloid precursor tryptophan was found to be a pathway regulator which induces alkaloid synthesis in fungal cultures, including cultures of Claviceps purpurea grown in a high phosphate medium where the pathway would normally be inhibited (Krupinski et al., 1976). Transcription factors are not encoded within the ergot alkaloid gene cluster; though there are several known transcription factors ( $\mathrm{Br} / \mathrm{A}$ and StuA) involved in regulating asexual development in Aspergillus spp. that are linked with ergot alkaloid production (Mulinti et al., 2014; Coyle et al., 2007). In Epichloë festucae expression of eas genes was found to be linked with histone methylation (H3K9 and H3K27) whereupon in planta cultures showed reduced methylation and altered chromatin structure compared to axenic cultures (Chujo and Scott, 2014). This finding provides insight as to why endophytes produce ergot alkaloids during plant infection rather than in culture.

\section{Gene Cluster Variation}

Ergot alkaloid synthesizing (eas) genes are localized within gene clusters, which are usually located close to telomeres (Schardl et al., 2013; Panaccione, 2010). The first eas cluster was found in $C$. purpurea by genome walking utilizing the dmaW gene as a reference (Tudzynski et al., 1999). Clusters in other organisms have since been found through genome sequencing and alignments. Genetic variation within cluster loci is noticeable between species and is responsible for the high level of diversity in the ergot alkaloid pathway (Figure 1, 2). Sequencing of eas clusters from several ergot producers revealed differences in gene arrangement, presence or absence of functional genes, and variations in coding sequences (Figure 1). Specific isolates may also contain more than one eas cluster embedded within the genome such as the hybrid Epichloë coenophiala (Florea et al., 2013). Several isolates of known alkaloid producers have been found to prematurely end progress through the pathway due to mutations within eas coding regions (Robinson and Panaccione, 2012; Schardl et al., 2013). Sequencing of multiple Epichloë species indicated that within this genus the ergot alkaloid cluster is not highly stable and exhibits drastic alterations between isolates (Schardl et al., 2013). Eas clusters have also been found in non-ergot alkaloid producing fungi in the Clavicipitaceae such as Metarhizium spp. (insect pathogens); however genome sequencing of Metarhizium anisopliae and M. acridum revealed mutations in eas 
genes causing loss of pathway function (Gao et al., 2011; Schardl et al., 2013). Sequencing of the human pathogen Microsporum (Arthrodermataceae family) indicated the presence of an eas cluster within the genome; nevertheless ergot alkaloids have not yet been reported in Microsporum spp.

Several eas genes contain orthologues found in most ergot alkaloid gene clusters; these genes are involved in the early-pathway to mid-pathway steps. For example, all clusters illustrated in Figure 1 contain seven eas orthologues: $d m a W$, eas $F$, easE, eas $C$, eas $A$, easD, easG. The presence of these seven genes will lead to the formation of either agroclavine or festuclavine, depending on which eas $A$ allele is present (Cheng et al., 2010; Coyle et al., 2010). Genes involved in the late stages of the pathway show a higher level of variation. A. fumigatus is located within the Trichocomaceae family, and therefore is distantly related to ergot alkaloid producers in the Clavicipitaceae (Claviceps, Epichloë, and Periglandula). Four genes, eas $M$, eas $N$, easL, and eas $K$, are specific to the $A$. fumigatus cluster and responsible for the formation of fumigaclavine end products (Bilovol and Panaccione, unpublished; Panaccione, 2010). On the other hand, several genes are specific to the Clavicipitaceae, such as IpsB and cloA, while other genes are species specific, such as eas $P$ and easO (Panaccione, 2010; Schardl et al., 2013).

\section{Early steps of the pathway}

Although there is much variation in alkaloid profiles among producers, the early steps in the pathway appear to be evolutionarily conserved (Figure 2) (Panaccione, 2010; Schardl et al., 2006). The prenylation of tryptophan by $4-\gamma, \gamma$-dimethylallyltryptophan (DMAT) synthase, also called DmaW, was found to be the determinant step of alkaloid synthesis producing the first intermediate DMAT (Lee et al., 1976; Wang et al. 2004; Coyle and Panaccione, 2005). DmaW was first isolated from Claviceps sp. DS 58 and found to convert substrates L-tryptophan and dimethlyallypyrophosphate (DMAPP) into DMAT and inorganic phosphate (Lee et al., 1976). The $d m a W$ gene was later discovered from C. purpurea via gene expression in a yeast hybrid system (Tsai et al., 1995). Gene alignments were used to identify an orthologue of $d m a W$ in the phylogenetically divergent fungus $A$. fumigatus, and further investigations revealed this orthologue was also responsible for DMAT synthesis (Unsöld and Li, 2005). The role of DmaW in alkaloid synthesis was further supported by knockout studies in two different fungi, $A$. fumigatus and Epichloë sp. isolate Lp1, which resulted in failure of both fungi to produce any detectable ergot alkaloids.

The easF gene encodes a methlytransferase which catalyzes the second biosynthetic reaction converting DMAT to N-me-DMAT (Rigbers and Li, 2008). The $A$. fumigatus eas $F$ gene was overexpressed in and purified from Escherichia coli and found to N-methylate DMAT using S-adenosylmethionine as a 
methyl donor. Alkaloid extracts from A. fumigatus easF knockout strains did not accumulate N-meDMAT which further supports the role of easF in N-me-DMAT formation (Coyle and Panaccione, 2008). The conversion of N-me-DMAT to chanoclavine-I is not completely understood, though both EasE and EasC are known to be required for chanoclavine-I synthesis by knockout studies (Goetz et al., 2011; Lorenz et al., 2010).

\section{Genetic Basis for late steps in the pathway}

After chanoclavine-I is oxidized to chanoclavine aldehyde catalyzed by EasD (Wallway et al., 2010a), the ergot alkaloid pathway begins to diverge (Figure 2). Genetic variations within the eas $A$ coding sequence have led to the formation of two EasA alleles (Cheng et al., 2010; Coyle et al., 2010). Fungi in the Trichocomaceae contain a version of EasA capable of fully reducing the 8, 9 carbon double of chanoclavine aldehyde which, followed by involvement of EasG, synthesizes festuclavine. On the other hand, producers in the Clavicipitaceae generally contain an easA allele that cannot serve as a proton donor for 8, 9 double bond reduction. This EasA variant, along with EasG, is responsible for agroclavine synthesis.

As previously discussed, $A$. fumigatus produces a different array of alkaloids compared to fungi in the Clavicipitaceae due to genetic variation. Four genes are unique to the $A$. fumigatus eas cluster (easM, eas $N$, easL, and easK), all of which are responsible for the formation of clavine alkaloids. It was recently discovered via knockout studies that eas $M$, encoding a monooxygenase, is responsible for oxidizing festuclavine at position 9 to form fumigaclavine B (Bilovol and Panaccione, not published). The Eas $N$ enzyme then acetylates fumigaclavine $B$ to yield fumigaclavine $A$ (Liu et al., 2009). Formation of endproduct fumigaclavine $C$ is catalyzed by a reverse prenlytransferase encoded by the eas $L$ gene (Unsöld and Li, 2006). The easK gene encodes an uncharacterized monooxygenase; though it is plausible this enzyme is responsible for the oxidation of an unknown alkaloid to yield fumigaclavine $D$.

Fungi in the Clavicipitaceae generally produce lysergic acid amides and ergopeptines using agroclavine as a precursor. The $c l o A$ gene codes for an oxidase responsible for two sequential oxidation reactions synthesizing elymoclavine followed by lysergic acid (Haarman et al., 2006; Robinson and Panaccione, 2014). Most alkaloid producers in this family possess the IpsB gene and some contain IpsC, which are both translated into lysergyl peptide synthetases. The LpsB and LpsC enzymes interact to convert lysergic acid to form the lysergic acid amide ergonovine (Ortel and Keller, 2009). Other producers may possess the IpsA gene that encodes a lysergyl peptide synthetase that interacts with LpsB to form ergopeptines with various amino acid side chains (Reiderer et al., 1996; Walzel et al., 1997).

\section{Project Objectives and Relevance}


The objectives of this study were to:

1. Determine if genes $d m a W$, easF, easE, and eas $C$ are sufficient to synthesize chanoclavine by expressing these genes in the non-ergot alkaloid producing fungus Aspergillus nidulans

This study will provide knowledge of the shared steps in ergot alkaloid formation.

Understanding the formation of ergot alkaloids, may allow for better modification of these compounds for clinical purposes and/or the ability to control their synthesis in agricultural settings. This study also provides a novel approach of understanding ergot alkaloid biosynthesis by heterologous expression in $A$. nidulans.

2. Investigate the roles of EasC and EasE by generating fungal transformants with subset combinations of the following genes dmaW, easF, easE, and easC.

The eas $C$ and eas $E$ genes have been difficult to study during in vitro studies and remain uncharacterized. This study will provide an alternative method to investigate the functions of both genes. In the process of generating transformants with altered combinations of the genes above, we can learn of intermediates produced in the pathway and potentially identify uncharacterized ergot alkaloids.

3. Investigate the involvement of easA in the formation of cycloclavine and lysergol.

Lysergol is a pharmaceutically important compound, and cycloclavine is a unique alkaloid possessing a high energy cyclopropane bond. This study may provide insight to the biosynthesis of both compounds.

4. Genetically modify endophytes for altered ergot alkaloid production and determine the involvement of eas $O$ and eas $P$ in LAH synthesis.

The focus of this study is to modify grass endophytes to produce ergonovine and potentially LAH. Ergonovine has bioprotective capabilities which may improve plant health; however most grass endophytes do not synthesize this compound. The development of these altered endophytes may allow for advanced use of ergot alkaloids in agricultural settings.

\section{References}

Ahimsa-Müller M, Markert A, Hellwig S, Knoop V, Steiner U, Drewke C, Leistner E. 2007. Clavicipitaceous fungi associated with ergoline alkaloid-containing Convolvulaceae. J. Nat. Prod. 70: 1955-1960.

Amici AM, Minghetti A, Scottl T, Spalla C, Tognoli L. 1969. Production of peptide ergot alkaloids in submerged culture by three isolates of Claviceps purpurea. Appl. Microbial. 18: 464-468.

Bacetty AA, Snook ME, Glenn AE, Noe FP, Hill N, Culbreath A, Timper P, Nagabhyru P, Bacon CW. 2009. toxicity of endophyte-infected tall fescue alkaloids and grass metabolites on Pratylenchus scribneri. Phytopath. 99: 1336-1345. 
Ball OJP, Bernard ED, Gwinn KD. 1997. Effect of selected Neotyphodium lolii isolates on root-knot nematode (Meloidogyne marylandi) number in perennial ryegrass. In Proceedings $50^{\text {th }}$ Plant Protection Conference, Manaaki Whenua Press, Lincoln, New Zealand, pp 65-68.

Beaulieu WT, Panaccione DG, Hazekamp CS, Mckee MC, Ryan KL, Clay K. 2013. Differential Allocation of seed-borne ergot alkaloids during early ontogeny of morning glories (Convolvulaceae). J. Chem. Ecol. 39: 919-930.

Belser-Ehrlich S, Harper A, Hussey J, Hallock R. 2013. Human and cattle ergotism since 1900: symptoms, outbreaks, and regulations. Toxicol. Ind. Health 29: 307-316.

Bouton JH, Latch G, Hill NS, Hoveland CS, McCann MA, Watson RH, Parish JA, Hawkins LL, Thompson FN. 2002. Reinfection of tall fescue cultivars with non-ergot alkaloid-producing endophytes. Agron. J. 94: 567-574.

Cano PM, Jamin EL, Tadrist S, Bourdaud'hau P, Pean M, Debrauwer L, Oswald IP, Delaforge M, Puel O. 2013. New untargeted metabolic profiling combining mass spectrometry and isotopic labeling: application on Aspergillus fumigatus grown on wheat. Anal. Chem. 85: 8412-20.

Cheng JZ, Coyle CM, Panaccione DG, O'Connor SE. 2010. Controlling a structural branch point in ergot alkaloid biosynthesis. J. Am. Chem. Soc. 132: 12835-12837.

Chujo T, Scott B. 2014. Histon H3K9 and H3K27 methylation regulates fungal alkaloid biosynthesis in a fungal endophyte-plant symbiosis. Mol. Microbiol. 92: 413-434.

Christensen MJ, Bennett RJ, Schmid J. 2002. Growth of Epichloë / Neotyphodium and p-endophytes in leaves of Lolium and Festuca grasses. Mycol. Res. 106: 93-106.

Clay K, Schardl C. 2002. Evolutionary origins and ecological consequences of endophyte symbiosis with grasses. Amer. Nat. 160: 99-127.

Code of Federal Regulations (CFR), Title 7, Section 810 - Agriculture, Official US Standards for Grain, Jan 1, 2001

Coyle CM, Panaccione DG. 2005. An ergot alkaloid biosynthesis gene and clustered hypothetical genes from Aspergillus fumigatus. Appl. Environ. Microbiol. 71: 3112-3118.

Coyle CM, Kenaley SC, Rittenour WR, Panaccione DG. 2007. Association of ergot alkaloids with conidiation in Aspergillus fumigatus. Mycologia 99: 804-811.

Coyle, C M, Goetz KE, Panaccione DG. 2008. Clustered genes common to both Aspergillus fumigatus and ergot fungi control early steps within the ergot alkaloid pathway. Phytopath. 98: 3340.

Coyle CM, Cheng JZ, O'Connor SE, Panaccione DG. 2010. An old yellow enzyme gene controls the branch point between Aspergillus fumigatus and Claviceps purpurea ergot alkaloid pathways. Appl. Environ. Microbiol. 76: 3898-3903.

Eadie, MJ. 2003. Convulsive ergotism: epidemics of the serotonin syndrome? Lancet Neuro. 2: 429-434.

Eich E. 2008. Solanaceae and Convolvulaceae secondary metabolites: Biosynthesis, chemotaxonomy, biological and economic significance. Springer, Berlin, Germany.

Eich E, Eichberg D, Muller W. 1984. Clavines. New antibiotics with cytostatic activity. Biochem. Pharmacol. 4: 523-526.

European Food Safety Authority. 2012. Scientific opinion on ergot alkaloids in food and feed, EFSA Panel on contaminants in the food chain. EFSA 10: 2798. 
Florea S, Farman ML, Panaccione DG, Yound CA, Schardl CL. 2013. Alteration of the ergot alkaloid profile through chromosome end knockoff. Phytopath. 103: 3340.

Furuta T, Koike M, Matazo A. 1982. Isolation of cycloclavine from the culture broth of Aspergillus japonicas SAITO. Agric. Biol. Chem. 46: 1921-1922.

Glasser A. 1961. Some pharmacological actions of D-lysergic acid methlycarbinolamine. Nature 189: 313314.

Gao Q, Jin K, Ying S-H, Zhang Y, Xiao G, Shang Y, Duan Z, Hu X, Xie X, Zhou G, Peng G, Luo Z, Huang W, Wang B, Fang W, Wang S, Zhong Y, Ma L, Leger R, Zhao G, Pei Y, Feng M, Xia Y, Wang C. 2011. Genome sequencing and comparative transcriptomics of the model entomopathogenic fungi Metarhizium anisopliae and M. acridum. PLOS Genetics 7: e1001264.

Goetz KE, Coyle CM, Cheng JZ, O'Conner S, Panaccione DG. 2011. Ergot cluster-encoded catalase is required for synthesis of chanoclavine-I in Aspergillus fumigatus. Curr. Genet. 57: 201-211.

Halpern JH. 2004. Hallucinogens and dissociative agents naturally growing in the United States. Pharmacol. Ther. 102: 131-138.

Haarman T, Machado C Lubbe Y, Correia T, Schardl CL, Panaccione DG, Tudzynski P. 2005. The ergot alkaloid gene cluster in Claviceps purpurea: extension of the cluster sequence and intra species evolution. Phytochem. 66: 1312-1320.

Haarmann T, Ortel I, Tudzynski P, Keller U. 2006. Identification of the cytochrome P450 monooxygenase that bridges the clavine and ergoline alkaloid pathways. Chem. Biochem. 7: 645-652.

Howard WF, McFadden PR, Keettel WC. 1964. Oxytocic drugs in fourth stage of labor. JAMA 189: 411413.

Hulvová H, Galuszka P, Frébortová J, Frébort I. 2013. Parasitic fungus Claviceps as a source for biotechnological production of ergot alkaloids. Biotechnol. Adv. 31: 79-89.

Ilha MR, loretti AP, Barros CS. 2003. Hyperthermic syndrome in dairy cattle associated with consumption of ergots of Claviceps purpurea in southern Brazil. Vet. Human Toxicol. 45: 140-145.

Janardhanan KK, Sattar A, Husain A. 1984. Production of fumigaclavine A by Aspergillus tamari Kita. Can. J. Microbiol. 30: 247-250.

Khanuja S, Arya JS, Srivastava SK, Shasany AK, Kumar S, Ranganathan T, Darokar MP, Kumar S. 2006. Antibiotic pharmaceutical composition with lysergol as bio-enhancer and method of treatment. United States Patent, Number 20070060604.

Krupinski VM, Robbers JE, Floss HG. 1976. Physiological study of ergot: induction of alkaloid synthesis by tryptophan at the enzymatic level. J. Bacteriol. 125: 158-165.

Kucht S, Groß J, Hussein Y, Grothe T, Keller U, Basar S, Konig W, Steiner U, Leistner E. 2004. Elimination of ergoline alkaloids following treatment of Ipomoea asarifolia (Convolvulaceae) with fungicides. Planta 219: 619-625.

Latgé JP. 1999. Aspergillus fumigatus and aspergillosis. Clin. Microbial. Rev. 12: 310-350.

Lee SL, Floss H, Heinstein P. 1976. Purification and properties of dimethylallylpyrophosphate: Tryptophan dimethylallyl transferase, the first enzyme of ergot alkaloid biosynthesis in Claviceps. sp. SD 58. Arch. Biochem. Biophysics. 177: 84-94. 
Leuchtmann A, Bacon CW, Schardl CL, White JF, Tadych M. 2014. Nomenclatural realignment of Neotyphodium species with genus Epichloe. Mycologia 106: 202-215.

Liu X, Wang L, Steffan N, Yin WB, Li SM. 2009. Ergot alkaloid biosynthesis in Aspergillus fumigatus: FgaAT catalyzes the acetylation of fumigaclavine B. Chem. BioChem. 10: 2325-2328.

Lorenz N, Olšovská J, Šulc M, Tudzynski P. 2010. Alkaloid cluster gene ccsA of the ergot fungus Claviceps purpurea encodes chanoclavine I synthase, a flavin adenine dinucleotide-containing oxidoreductase mediating the transformation of $\mathrm{N}$-methyl-dimethylallyltryptophan to chanoclavine. Appl. Environ. Microbiol.76: 1822-1830.

Maurya A, Dwivedi GR, Darokar MP, Srivastava SK. 2013. Antibacterial and synergy of clavine alkaloid lysergol and its derivatives against nalidixic acid-resistant Escherichia coli. Chem. Biol. Drug Des. 81: 484-490.

Merkel S, Dib B, Maul R, Koppen R, Koch M, Nehls I. 2012. Degradation and epimerization of ergot alkaloids after baking and in vitro digestion. Anal. Bioanal. Chem. 404: 2489-2497.

Mulinti P, Allen NA, Coyle CM, Gravelat FN, Sheppard DC, Panaccione DG. 2014. Accumulation of ergot alkaloids during conidiophore development in Aspergillus fumigatus. Curr. Microbiol. 68: 1-5.

Nemeth E. 199. Parasitic production of ergot alkaloids. In: Medicinal and aromatic plant-industrial profiles. Ergot, the genus Claviceps. (Eds. Kren V, Cvak L). Harwood Academic, Chur, pp 303-319.

O'Gorman CM, Fuller J, Dyer PS. 2009. Discovery of a sexual cycle in the opportunistic fungal pathogen Aspergillus fumigatus. Nature 457: 471-474.

Ortel I, Keller U. 2009. Combinatorial assembly of simple and complex D-lysergic acid alkaloid peptide classes in the ergot fungus Claviceps purpurea. J. Biol. Chem. 284: 6650-6660.

Panaccione DG, Johnson RD, Wang J, Young CA, Damrongkool P, Scott B, Schardl CL. 2001. Elimination of ergovaline from a grass-Neotyphodium endophyte symbiosis by genetic modification of the endophyte. Pro. Natl. Acad. Sci. USA 98: 12820-12825.

Panaccione DG, Tapper BA, Lane GA, Davies E, Fraser K. 2003. Biochemical outcome of blocking the ergot alkaloid pathway of a grass endophyte. J. Agric. Food. Chem. 51: 6429-6437.

Panaccione DG. 2005. Origins and significance of ergot alkaloid diversity in fungi. FEMS Microbiol. Lett. 251: 9-17.

Panaccione DG, Coyle CM. 2005. Abundant respirable ergot alkaloids from the common airborne fungus Aspergillus fumigatus. Appl. Environ. Microbial. 71: 3106-3111.

Panaccione DG, Cipoletti JR, Sedlock AB, Blemings KP, Schardl CL, Machado C, Seidel GE. 2006a. Effects of ergot alkaloids of food preference and saiety in rabbits, as assessed with gene knockout endophytes in perennial ryegrass (Lolium perenne). J. agric. Food. Chem. 54: 4582-4587.

Panaccione DG, Kotcon JB, Schardl CL, Johnson RJ, Morton JB. 2006b. Ergot alkaloids are not essential for endophytic fungus assocaiated population suppression of the lesion nematode, Pratylenchus scribneri, on perennial ryegrass. Nematol. 8: 583-590.

Panaccione DG. 2010. Ergot alkaloids. The Mycota, Vol. X, Industrial Applications, 2nd Edition (Ed. M. Hofrichter), Berlin-Heidelburg: Springer-Verlag, 195-214. 
Parish JA, McCann MA, Watson RH, Paiva NN, Hoveland CS, Parks AH, Upchurch BL, Hill NS, Bouton JH. 2003. Use of nonergot alkaloid-producing endophytes for alleviating tall fescue toxicosis in stocker cattle. J. Anim. Sci. 81: 2856-2868.

Passie T, Jalpern JH, Stichtenth DO, Emrich HM, Hintzen A. 2008. The pharmacology of lysergic acid diethylamide: a review. CNS Neurosci. Ther. 14: 295-314.

Pertz H, Eich E. 1999. Ergot alklaloids and their derivatives as ligands for serotoninergic, dopaminergic, and adrenergic receptors. In: kren V, Cvak L (eds) Ergot: the genus Claviceps. Harwood, Rotterdam, pp 411-440.

Pertz H. 1996. Naturally occurring clavines: antagonism/partial agonism at 5- $\mathrm{Ht}_{2 \mathrm{a}}$ receptors and antagonism at $\alpha 1$-adrenoceptors in blood vessels. Planta Med. 62: 387-392.

Potter DA, Stokes JT, Redmond CT, SchardI CL, Panaccione DG. 2008. Contribution of ergot alkaloids to suppression of a grass-feeding caterpillar assessed with gene knockout endophytes in perennial ryegrass. Entom. Exp. App. 126: 138-147.

Riederer B, Han M, Keller U. 1996. D-Lysergyl peptide synthetase from the ergot fungus Claviceps purpurea. J. Biol. Chem. 271: 27524-27530.

Rigbers O, Li SM. 2008. Ergot alkaloid biosynthesis in Aspergillus fumigatus overproduction and biochemical characterization of a 4-dimethylallyltryptophan N-methyltransferase. J. Biol. Chem. 283: 26859-26868.

Roa KK, Gupta AR, Singh VK. 1977. Effect of phosphate on ergot alkaloid synthesis in Aspergillus fumigatus. Folia Microbiol. (Praha) 22: 145-419.

Robbers JE, Robertson LW, Hornemann KM, Jindra A, Floss HG. 1972. Physiological studies on ergot: further studies on the induction of alkaloid synthesis by tryptophan and its inhibition by phosphate. J. Bacter. 112: 791-796.

Robinson SL, Panaccione DG. 2012. Chemotypic and genotypic diversity in the ergot alkaloid pathway of Aspergillus fumigatus. Mycologia 104: 804-812.

Robinson S, Panaccione DG. 2014. Heterologous expression of lysergic acid and novel ergot alkaloids in Aspergillus fumigatus. Appl. Environ. Microbiol. 80: 6465-6472.

Schardl CL, Young CA, Hesse U, Amyotte SG, Andreeva K, Calie PJ, Fleetwood DJ, Haws DC, Moore N, Oeser B, Panaccione DG, Schweri KK, Voisey CR, Farman ML, Jaromczyk JW, Roe BA, O'Sullivan DM, Scott B, Tudzynski P, An Z, Arnaoudova EG, Bullock CT, Charlton ND, Chen L, Cox M, Dinkins RD, Florea S, Glenn AE, Gordon A, Güldener U, Harris DR, Hollin W, Jaromczyk J, Johnson RD, Khan AK, Leistner E, Leuchtmann A, Li C, Liu JG, Liu J, Liu M, Mace W, Machado C, Nagabhyru P, Pan J, Schmid J, Sugawara K, Steiner U, Takach JE, Tanaka E, Webb JS, Wilson EV, Wiseman JL, Yoshida RY, Zeng Z. 2013. Plant-symbiotic fungi as chemical engineers: multi-genome analysis of the Clavicipitaceae reveals dynamics of alkaloid loci. Plos Genetics 9: e1003323.

Schardl CL, Phillips TD. 1997. Protective grass endophytes: Where are they from and where are they going? Plant Dis. 81: 430-437.

Schardl CL, Leuchtmann A, Spiering MJ. 2004. Symbioses of grasses with seedborne fungal endophytes. Annu. Rev. Plant Biol. 55: 315-340.

Schneider HR, Stadler PA, Stuetz P, Troxler F, Seres J. 1977. Synthesis and properties of bromocriptine. Experiential 33: 1412-1413. 
Schultes RE, Hofmann A. 1992. Plants of the Gods: Their sacred, healing and hallucinogenic powers. Rochester,VT: Healing Arts Press.

Scudamore KA, Livesey CT. 1997. Occurrence and significance of mycotoxins in forage crops and silage: a review. J. Sci. Food Agric. 77: 1-17.

Steiner U, Ahimsa-Muller MA, Markert A, Kucht S, Groß J, Kauf N, Kuzma M, Zych M, Lamsho“ ft M, Furmanowa M, Knoop V, Drewke C, Leistner E. 2006. Molecular characterization of a seed transmitted clavicipitaceous fungus occurring on dicotyledonous plants (Convolvulaceae). Planta 224: 533-544.

Steiner U, Leibner S, SchardI CL, Leuchtmann A, Leistner E. 2011. Periglandula, a new fungal genus within the Clavicipitaceae and its association with Convolvulaceae. Mycologia 103: 1133-1145.

Tfelt-Hansen PC, Koehler PJ. 2008. History of the use of ergotamine and dihydroergotamine in migraine from 1906 and onward. Cephalalgia 28: 877-886.

Timper P, Gates RN, Bouton JH. 2005. Response of Pratylenchus spp. in tall fescue infected with different strains of the fungal endophyte Neotyphodium coenophialum. Nematol. 7: 105-110.

Tsai H-F, Wang H, Gebler JC, Poulter CD, Schardl CL. 1995. The Claviceps purpurea gene encoding dimethylallyltryptophan synthase, the committed step for ergot alkaloid biosynthesis. Biochem. Biophys. Res. Comm. 216: 119-125.

Tudzynski P, Holter K, Correia T, Arntz C, Grammel N, Keller U. 1999. Evidence for an ergot alkaloid gene cluster in Claviceps purpurea. Mol. Gen. Genet. 261: 133-141.

Tudzynkski P, Correia T, Keller U. 2001. Biotechnology and genetics of ergot alkaloids. Appl. Microbiol. Biotechnol. 57: 593-605.

Tudzynski P, Scheffer J. 2004. Claviceps purpurea: molecular aspects of a unique pathogenic lifestyle. Mol. Plant Path. 5: 377-388.

United States Codes, Title 21, Chapter 13, Controlled Substances ACT- Control and Enforcement, Oct 27, 1970

United States Food and Drug Administration. 2007. Public Health Advisory, 29 March. http://www.fda.gov/Drugs/DrugSafety/PublicHealthAdvisories/ucm051285.html

Unsöld AI, Li SM. 2005. Overproduction, purification and characterization of FgaPT2, a dimethylallyltryptophan synthase from Aspergillus fumigatus. Microbiol. 115: 1499-1505.

Unsöld Al, Li SM. 2006. Reverse prenyltransferase in the biosynthesis of fumigaclavine C in Aspergillus fumigatus: gene expression, purification, and characterization of fumigaclavine $C$ synthase FGAPT1. Chem. Bio. Chem. 7: 158-164.

Urga K, Debella A, Medihn Y, Agata N, Bayu A, Zewdie W. 2002. Laboratory studies on the outbreak of gangrenous ergotism associated with consumption of contaminated barley in arsi, Ethiopia. Ethiopian J. Health Devel. 16: 317-323.

Vining LC, Taber WA. 1959. Estimation of ergot alkaloids in cultures of Claviceps purpurea. Can J. Microbial. 5: 441-451.

Wallwey C, Matuschek M, Li SM. 2010a. Ergot alkaloid biosynthesis in Aspergillus fumigatus: conversion of chanoclavine-I to chanoclavine-I aldehyde catalyzed by a short-chain alcohol dehydrogenase FgaDH. Arch. Microbiol. 192: 127-34. 
Wallwey C, Matuschek M, Xie X-L, Li SM. 2010b. Ergot alkaloid biosynthesis in Aspergillus fumigatus: Conversion of chanoclavine-I aldehyde to festuclavine by the festuclavine synthase FgaFS in the presence of the old yellow enzyme FgaOx3 Org. Biomol. Chem. 8: 3500-3508.

Walzel B, Riederer B, Keller U. 1997. Mechanism of alkaloid cyclopeptide synthesis in the ergot fungus Claviceps purpurea. Chem. Biol. 4: 223-230.

Wang J, Machado C, Panaccione DG, Tsai H-F, Schardl CL. 2004. The determinate step in ergot alkaloid biosynthesis by an endophyte of perennial ryegrass. Fungal Genet. Biol. 41: 189-198.

Watanabe H, Somei M, Sekihara S, Nakagawa K, Yamada F. 1987. Dopamine receptor stimulating effects of chanoclavine analogues, tricyclic ergot alkaloids, in the brain. Japan J. Pharmacol. 45: 501506.

Wu XF, Fei MJ, Shu RG, Tan RX, Xu Q. 2005. Fumigaclavine C, a fungal metabolite, improves experimental colitis in mice via downregulating Th1 cytokine production and matrix metalloproteinase activity. Int. Immunopharmacol. 5: 1543-1553.

Zanettini R, Antonini A, Gatto G, Gentile R, Tesei S, Pezzoli G. 2007. Valvular heart disease and the use of dopamine agonists for Parkinson's disease. N. Engl. J. Med. 356: 39-46.

Zhao Y, Liu J, Wang J, Wang I, Yin H, Tan R, Xu Q. 2004. Fumigaclavine C improves concanavalin Ainduced liverinjury in mice mainly via inhibiting TNF-a productionand lymphocyte adhesion to extracellular matrices. J. Pharm. Pharmacol. 56: 775-780. 


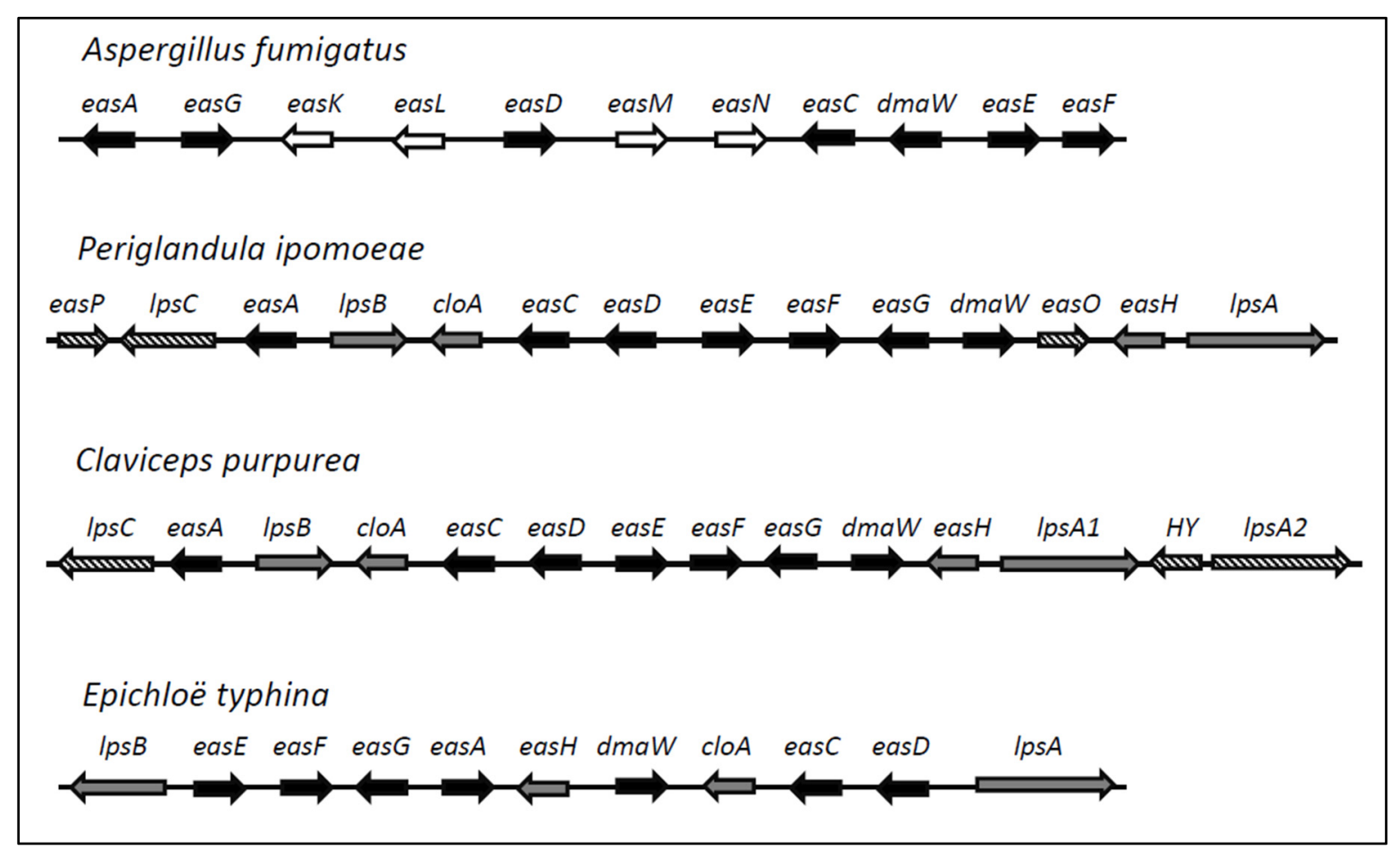

Figure 1: Ergot alkaloid gene clusters from different fungi. Directional arrows indicate gene orientation. Genes shown in black are common in all clusters; genes in white are specific to $A$. fumigatus; genes shown in gray and cross-hatched are specific to Clavicipitaceae. Clusters are redrawn from Panaccione, 2010 and Schardl et al., 2013 


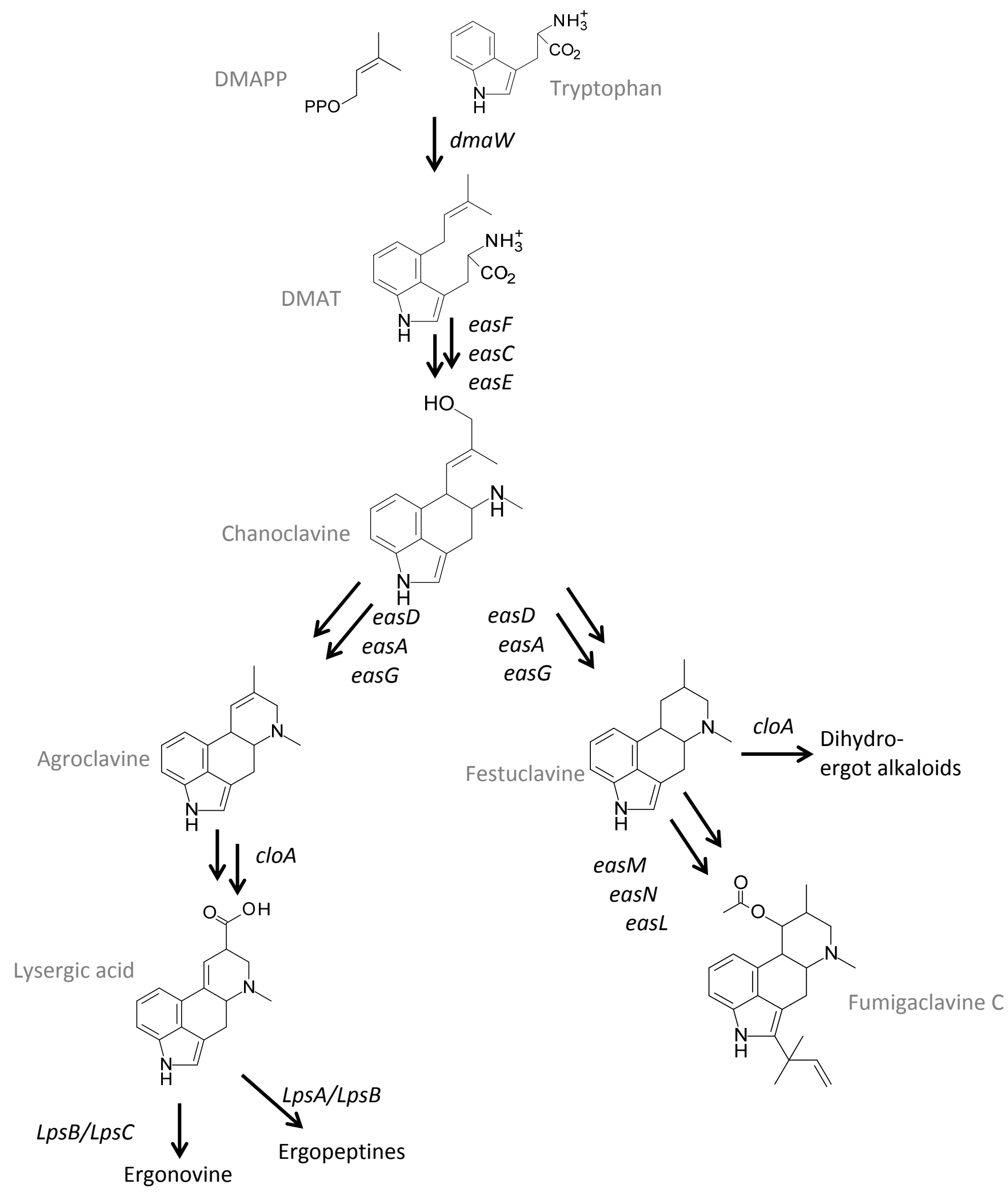

Figure 2: Ergot alkaloid pathway. For conciseness, some intermediates are not shown. Gene names are listed next to arrows at involved steps. Pathway is redrawn from Panaccione, 2010. DMAPP= Dimethylallylpyrophosphate, DMAT= Dimethylallytryptophan 


\section{Chapter 2}

Partial Reconstruction of the Ergot Alkaloid Pathway by Heterologous Gene Expression in Aspergillus nidulans

Katy L. Ryan, Christopher T. Moore and Daniel G. Panaccione

Published in Toxins 2013, 5: 445-455; doi:10.3390/toxins5020445 


\section{Abstract}

Ergot alkaloids are pharmaceutically and agriculturally important secondary metabolites produced by several species of fungi. Ergot alkaloid pathways vary among different fungal lineages, but the pathway intermediate chanoclavine-I is evolutionarily conserved among ergot alkaloid producers. At least four genes, dmaW, easF, easE, and easC, are necessary for pathway steps prior to chanoclavine-l; however, the sufficiency of these genes for chanoclavine-I synthesis has not been established. A fragment of genomic DNA containing $d m a W$, eas $F, e a s E$, and eas $C$ was amplified from the humanpathogenic, ergot alkaloid-producing fungus Aspergillus fumigatus and transformed into Aspergillus nidulans, a model fungus that does not contain any of the ergot alkaloid synthesis genes. HPLC and LCMS analyses demonstrated that transformed $A$. nidulans strains produced chanoclavine-I and an earlier pathway intermediate. Aspergillus nidulans transformants containing $d m a W$, eas $F$, and either easE or eas $C$ did not produce chanoclavine-I but did produce an early pathway intermediate and, in the case of the eas $C$ transformant, an additional ergot alkaloid-like compound. We conclude that $d m a W$, eas $F$, easE, and eas $C$ are sufficient for the synthesis of chanoclavine-I in $A$. nidulans and expressing ergot alkaloid pathway genes in $A$. nidulans provides a novel approach to understanding the early steps in ergot alkaloid synthesis.

\section{Introduction}

Ergot alkaloids are tryptophan-derived mycotoxins produced by several different fungi and have proven to be both pharmaceutically and agriculturally important. Several Claviceps, Neotyphodium, and Epichloë species of the order Hypocreales produce a variety of complex ergot alkaloids (Lorenz et al., 2009; Panaccione, 2010). Aspergillus fumigatus and Penicillium commune of the order Eurotiales also are capable of ergot alkaloid synthesis; however, their ergot alkaloid profiles differ considerably from those of the Hypocrealean ergot alkaloid producers (Panaccione, 2010; Wallwey and Li, 2011). Ergot alkaloid-producing fungi contain an ergot alkaloid synthesis (eas) gene cluster with a set of core genes that is well conserved (Lorenz et al., 2009; Panaccione, 2010; Schardl et al., 2013). The eas clusters of the various ergot alkaloid producers also contain variable, lineage-specific genes that result in the formation of different pathway products among lineages (Panaccione, 2010; Schardl et al., 2013). The steps of ergot alkaloid synthesis leading to the formation of chanoclavine-I (the earliest ergot alkaloid pathway biosynthetic intermediate observed accumulating in most producers) (Figure 1) are believed to be evolutionarily conserved among the different fungi that produce ergot alkaloids (Lorenz et al., 2009; 
Panaccione, 2010; Schardl et al., 2013); however, the identity and catalytic activities of all the genes required for chanoclavine-I synthesis have not yet been determined.

Based on the proposed biochemical scheme of Kozikowski et al. (1993), the synthesis of chanoclavine-l occurs in at least five steps. Gene knockout and heterologous expression studies have demonstrated that at least four genes, $d m a W$, eas $F$, eas $E$, and eas $C$, are required prior to chanoclavine-I (Wang et al., 2004; Rigbers and Li, 2008; Lorenz et al., 2010; Goetz et al., 2011). However, it is currently unknown whether these genes are sufficient to synthesize chanoclavine-I. DMAT synthase, the product of $d m a W$, catalyzes the initial prenylation reaction to yield dimethylallyltryptophan (DMAT) (Wang et al., 2004; Unsöld and Li, 2005; Coyle and Panaccione, 2005), and easF encodes a methyltransferase that $N$ methylates DMAT to form the second intermediate, $N$-Me-DMAT (Rigbers and Li, 2008). The order of activity and function of EasE and EasC have not been established, though gene knockout studies indicate these enzymes are necessary for the conversion of N-Me-DMAT to chanoclavine-I (Lorenz et al., 2010; Goetz et al., 2011). Based on their sequences, easE encodes an oxidoreductase and eas $C$ encodes a catalase. Involvement of additional enzymes cannot be excluded because the synthesis of chanoclavineI requires two two-electron oxidations, and EasE is the only oxidoreductase among the four required genes. However, the eas clusters in the diverse fungi that produce ergot alkaloids do not contain any additional conserved oxidoreductase genes for which a function has not been assigned. Thus, the question of sufficiency of the four required genes is unresolved.

Aspergillus fumigatus is a common saprophytic fungus and potentially fatal pathogen in immunocompromised individuals (Latgé, 1999). Ergot alkaloid synthesis genes are clustered in the $A$. fumigatus genome (Unsöld and Li, 2005; Coyle and Panaccione, 2005) and are expressed during asexual reproduction (Coyle et al., 2007; Twumasi-Boateng et al., 2009). Aspergillus nidulans, a model fungus that is closely related to $A$. fumigatus, does not contain the ergot alkaloid gene cluster nor does it produce ergot alkaloids. The primary objective of this study was to test if $d m a W$, easF, eas $C$, and easE are sufficient to direct chanoclavine-I synthesis in $A$. nidulans. We used a novel approach to test the sufficiency of the genes by transforming $A$. nidulans with $d m a W$, eas $F$, eas $C$, and eas $E$, or subsets of those genes, from $A$. fumigatus under the control of their native promoters.

\section{Methods}

Fungal Strains and Growth Conditions: Aspergillus fumigatus isolate Af293, as a source of genomic DNA, was grown in malt extract broth ( $15 \mathrm{~g}$ malt extract/L) overnight at $37^{\circ} \mathrm{C}$. The uridine auxotrophic $A$. nidulans isolate FGSC A767 (Fungal Genetic Stock Center, Kansas City, MO, USA) was grown at $37{ }^{\circ} \mathrm{C}$ on 
SYE medium (20 g/L sucrose, $10 \mathrm{~g} / \mathrm{L}$ yeast extract, $10 \mathrm{~g} / \mathrm{L}$ MgSO4, $2 \mathrm{~mL} / \mathrm{L}$ trace element solution (Hunter et al., 1950), $2.2 \mathrm{~g} / \mathrm{L}$ uracil, $2.2 \mathrm{~g} / \mathrm{L}$ uridine, with or without $15 \mathrm{~g} / \mathrm{L}$ agar). For preparation of protoplasts, A. nidulans was grown in liquid SYE medium overnight. Transformants were regenerated on selective SYE agar medium which did not contain uracil or uridine and was supplemented with $304 \mathrm{~g} / \mathrm{L}$ sucrose (to serve as osmoticum as well as carbon source). To test alkaloid accumulation, cultures were grown on SYE agar medium at $37^{\circ} \mathrm{C}$ for one week.

DNA Manipulations: DNA was extracted from A. fumigatus according to the protocol described by Richards et al. (2001). An 8774-bp fragment containing dmaW, easF, easC, and easE was amplified by PCR primed with oligonucleotides primer 1 ( ${ }^{\prime}$ TACCTATACCTAATCGAAGCCGCACGCAGTGCACC ${ }^{3}$ ) and primer $2\left({ }^{5}\right.$ GCCGCCCATTCACCAAGATTTTTGCACAAATCTGCG $\left.{ }^{3}\right)$. A $25 \mu$ L reaction contained $1 \times$ LongAmp Taq reaction buffer, $200 \mu \mathrm{M}$ of each deoxynucleotide triphosphate, $1 \mu \mathrm{M}$ of each primer, and 2.5 units LongAmp Taq DNA polymerase (New England BioLabs, Ipswich, MA, USA). The reaction began with an initial denaturing step of $3 \mathrm{~min}$ at $94^{\circ} \mathrm{C}$, followed by 35 cycles of $30 \mathrm{~s}$ at $94{ }^{\circ} \mathrm{C}$ and $10 \mathrm{~min}$ at $65{ }^{\circ} \mathrm{C}$, with a final extension of $5 \mathrm{~min}$ at $65^{\circ} \mathrm{C}$. A 6123-bp fragment containing dmaW, eas $F$, and eas $E$ was amplified by PCR primed with oligonucleotides primer $3\left({ }^{5}\right.$ GAGAGCTACTTGACATATTGTGTCGGCAGGTGCGCA $\left.{ }^{3}\right)$ and primer $2\left({ }^{5}\right.$ GCCGCCCATTCACCAAGATTTTTGCACAAATCTGCG $\left.{ }^{3}\right)$. The PCR conditions were the same as stated above except the elongation time was $7 \mathrm{~min}$.

In order to generate transformant WFC (containing $d m a W$, eas $F$, and eas $C$ ), two separate DNA fragments were amplified for co-transformation. The first fragment containing $d m a W$ and eas $C$ was primed with primer $1\left(5^{\prime}\right.$ TACCTATACCTAATCGAAGCCGCACGCAGTGCACC $\left.{ }^{3}\right)$ and primer 5 (5' CTACTCCATTGTCCTGTGAGTTG ${ }^{3}$ ) which primed amplification of a 4846-bp fragment. The PCR conditions were the same as stated above except the elongation time was $5 \mathrm{~min}$. The second fragment (2943 bp) contained only eas F and was amplified by PCR primed with oligonucleotides easFF ( ${ }^{5}$ TCCATTCTTCGCTCGTTCAACCAGCAGG ${ }^{3}$ ) and easFR ( ${ }^{5}$ CAGGACCTGTACCTAAAGCCTGGTAACC $\left.{ }^{3}\right)$ in a $25 \mu$ teaction containing $1 \times$ GoTaq Flexi buffer, $200 \mu \mathrm{M}$ each deoxynucleotide triphosphate, $1.5 \mathrm{mM} \mathrm{MgCl} 2,1 \mu \mathrm{M}$ of each primer, and 2.5 units of Taq DNA polymerase (Promega, Madison, WI, USA). The reaction began with an initial denaturing step of $3 \mathrm{~min}$ at $94{ }^{\circ} \mathrm{C}$, followed by 35 cycles of $30 \mathrm{~s}$ at $94{ }^{\circ} \mathrm{C}, 30 \mathrm{~s}$ at $55^{\circ} \mathrm{C}$, and $3 \mathrm{~min}$ at $72{ }^{\circ} \mathrm{C}$, with a final extension of $5 \mathrm{~min}$ at $72{ }^{\circ} \mathrm{C}$.

PCR products were purified with QIAquick gel extraction kits (Qiagen, Valencia, CA, USA) prior to their inclusion in fungal transformations. The selectable marker pPyrG was obtained from the Fungal Genetics Stock Center and was digested with Xhol (New England BioLabs, Ipswich, MA, USA) and purified with QIAquick gel extraction kit prior to transformation. 
Fungal Transformation and Screening: Protoplasts of $A$. nidulans FGSC A767 were prepared by incubating overnight cultures with 50 mg driselase (Sigma-Aldrich, St. Louis, MO, USA), which was filter sterilized with a $0.22 \mu \mathrm{m}$ filter, and $75 \mathrm{mg}$ lysing enzyme (Sigma-Aldrich, St. Louis, MO, USA) in $15 \mathrm{~mL}$ of 0.7 M sodium chloride. Protoplasts were purified and co-transformed with PCR product and selectable marker (pPyrG) as described by Coyle et al. (2010). Protoplasts were plated on SYE agar medium containing no uracil or uridine. Transformation plates were incubated at $37^{\circ} \mathrm{C}$, and transformants were collected approximately three days after transformation. Each transformant was screened by PCR for the presence or absence of $d m a W$, eas $F, e a s C$, and easE. An internal region of $d m a W$ was primed with dmaWf ( ${ }^{\prime}$ TTGATCTGGAGTGGTTCCGC ${ }^{3}$ ) and dmaWR ( ${ }^{5}$ ' CGTTCATGCCGAAGGTTGTG ${ }^{3}$ ) yielding a 651-bp fragment. Primers for amplifying an internal region of easE were easEF ( ${ }^{5}$ CCAGATACATTGCCATCGCATG ${ }^{3}$ ) and easER ( (' TGTTCCAACTGCTTGGCCAGAT $^{3}$ ), which primed amplification of a 897-bp fragment. The presence or absence of eas $C$ was tested by PCR with primers easCF $\left({ }^{5}\right.$ GAATTCGAGGTATTGATCTCC $\left.{ }^{3}\right)$ and easCR ( ${ }^{5}$ AGCCAGGCAAAGATCCATAGTT ${ }^{3}$ ) which flank a 1036-bp fragment. An internal region of easF was primed with easFF ( ${ }^{\prime}$ AAGTTGTCGAAGGTCTCACGAA $\left.{ }^{3}\right)$ and easFR $\left({ }^{5}\right.$ GTGATTAGAGATGCTT$\mathrm{CTGTC}^{3}$ ), yielding an 820-bp fragment. Each of these PCRs were $25 \mu \mathrm{L}$ reactions containing $1 \times$ GoTaq Flexi buffer, $200 \mu \mathrm{M}$ deoxynucleotide triphosphate, $1.5 \mathrm{mM} \mathrm{MgCl}$, $1 \mu \mathrm{M}$ of each primer, and 2.5 units of Taq DNA polymerase (Promega, Madison, WI, USA). PCR conditions began with an initial denaturing step of 3 min at $94{ }^{\circ} \mathrm{C}$, followed by 35 cycles of $30 \mathrm{~s}$ at $94{ }^{\circ} \mathrm{C}$ followed by $30 \mathrm{~s}$ at $55^{\circ} \mathrm{C}$, and $1 \mathrm{~min}$ and $30 \mathrm{~s}$ at $72{ }^{\circ} \mathrm{C}$, followed by a final extension of $5 \mathrm{~min}$ at $72{ }^{\circ} \mathrm{C}$.

HPLC Analyses: Transformants positive for the presence of the target genes were analyzed by HPLC for the accumulation of chanoclavine-I. Ergot alkaloids were extracted by incubating a spore-rich sample of the colony, containing approximately $1 \mathrm{~cm} 2$ of culture surface area, in $400 \mu \mathrm{L}$ of methanol for $30 \mathrm{~min}$ and clarifying the extract by centrifugation. Extracts were injected into a C18 column (Prodigy $5-\mu m$ ODS3 (150 mm by $4.6 \mathrm{~mm}$ ); Phenomenex, Torrance, CA, USA) and subjected to a multilinear binary gradient from $5 \%(v / v)$ acetonitrile plus $95 \%(v / v)$ aqueous $50 \mathrm{mM}$ ammonium acetate to $75 \%$ acetonitrile plus $25 \%$ aqueous $50 \mathrm{mM}$ aqueous ammonium acetate at a flow rate $1 \mathrm{~mL} / \mathrm{min}$, as previously described by Panaccione et al. (2012). Ergot alkaloids were detected using two fluorescence settings. The first setting had an excitation wavelength of $272 \mathrm{~nm}$ and emission wavelength of $372 \mathrm{~nm}$; the second setting was $310 \mathrm{~nm}$ (excitation) and $410 \mathrm{~nm}$ (emission). Chanoclavine-I (generously provided by Brian Tapper, AgResearch, New Zealand) and N-Me-DMAT (gift from Sarah O'Connor, University of East Anglia) were run as standards. Peaks of interest were isolated from the HPLC by collecting the runoff from the discard tube for $30 \mathrm{~s}$ after the peak was visualized on the detector. 
LC-MS Analyses: Ergot alkaloid extracts were prepared by washing the surface of a conidiating colony with $2 \mathrm{~mL}$ of methanol, rotating the extract at $30 \mathrm{rpm}$ for $30 \mathrm{~min}$, and then clarifying the extract by centrifugation. Clarified extracts were then concentrated to a volume of $50 \mu \mathrm{L}$ in a speed-vac apparatus, and $10 \mu \mathrm{L}$ of concentrated extract were injected into the LC-MS. To isolate individual analytes, concentrated extracts were analyzed by fluorescence HPLC, peaks were collected manually, and concentrated to a volume of $50 \mu \mathrm{L}$ in a speed-vac apparatus prior to LC-MS analysis. Concentrated samples were analyzed on a Finnigan LCQDecaXP plus mass spectrometer equipped with a Surveyor HPLC system. Analytes were separated on a C18 column (Phenomenex 4- $\mu \mathrm{m}$ polar-RP (150 mm by 2 $\mathrm{mm}$ )), maintained at $30^{\circ} \mathrm{C}$, by combining mobile phases $\mathrm{A}(5 \%$ acetonitrile, $0.1 \%$ formic acid) and $\mathrm{B}(75 \%$ acetonitrile, $0.1 \%$ formic acid). Initial conditions were $86 \% A+14 \%$ B, which ramped linearly to $73 \% A+$ $27 \% \mathrm{~B}$ at $25 \mathrm{~min}, 25 \% \mathrm{~A}+75 \% \mathrm{~B}$ at $40 \mathrm{~min}$, and $0 \% \mathrm{~A}+100 \% \mathrm{~B}$ at $42 \mathrm{~min}$. The flow rate was $200 \mu \mathrm{L} / \mathrm{min}$. Analytes were ionized by electrospray ionization in positive mode and were detected by scanning for ions with a $m / z$ between $200-400$.

\section{Results}

\section{Engineering of Chanoclavine-I Producing Strains of $A$. nidulans.}

An 8.8-kb fragment of $A$. fumigatus DNA containing the genes $d m a W$, eas $F$, eas $E$, and eas $C$ was successfully amplified by PCR and integrated into the genome of two $A$. nidulans transformants, yielding strains WFEC33 and WFEC35. The presence of each of the four A. fumigatus genes in the WFEC strains was verified by PCR primed from internal gene primer-annealing sites (Figure 2). The recipient strain $A$. nidulans A767 did not contain any of the four genes. The absence of the ergot alkaloid gene cluster in $A$. nidulans was further supported by the lack of sequences with an E value less than 10 in a BLASTn search between the $A$. fumigatus dmaW, easF, easE, and eas $C$ cluster sequence with the genome of $A$. nidulans FGSC A4.

Both WFEC strains and A. nidulans A767 were analyzed by HPLC with fluorescence detection to investigate the presence of ergot alkaloids. Transformed strains accumulated an analyte that co-eluted with chanoclavine-I at approximately $33 \mathrm{~min}$, whereas $A$. nidulans A767 did not (Figure 3). Extracts of WFEC35 and $A$. nidulans A767 also were analyzed by electrospray ionization (ESI) LC-MS in positive mode. Again WFEC35 contained a peak that co-eluted with chanoclavine-I standard. The analyte had an $\mathrm{m} / \mathrm{z}$ of 257.1 consistent with the $[\mathrm{M}+\mathrm{H}]+$ of chanoclavine-I (Figure 4). HPLC with fluorescence detection of mutant strain WFEC35 also revealed the presence of the early pathway intermediate $N$-Me-DMAT which eluted at approximately $44 \mathrm{~min}$ (Figure 3 ). The identity of this analyte as $\mathrm{N}$-Me-DMAT was 
supported by an ion of $m / z 287.1$ in ESI LC-MS analyses (corresponding to [ $N$-Me-DMAT + H]+) and by co-elution with authentic standard (Figure 4).

Conidiating cultures produced modest quantities of chanoclavine-I. Aspergillus nidulans strain WFEC35 produced approximately $0.3 \mathrm{fg}$ chanoclavine-I per conidium, or about $3 \mathrm{ng}$ chanoclavine-I per $\mathrm{mm} 2$ of culture surface area and comparable quantities of $N$-Me-DMAT. Strain WFEC33 produced lesser quantities of chanoclavine-I and did not accumulate measurable quantities of $N$-Me-DMAT.

\section{Engineering of $\boldsymbol{A}$. nidulans Strains Expressing Subsets of Early Pathway Genes.}

Two additional mutant strains of $A$. nidulans were generated by transformation with subsets of three of the four ergot alkaloid cluster genes: strains WFC (containing $d m a W$, eas $F$, and eas $C$ ) and WFE (containing $d m a W, e a s F$, and easE). The presence of each gene in the genomes of the different mutants was verified by PCR (Figure 2). Chanoclavine-I was not detectable by HPLC (Figure 5) or ESI LC-MS (data not shown) in either strain. Expression of $d m a W$ and eas $F$ from the inserted construct in both strains was validated by the accumulation of the early pathway intermediate, $N$-Me-DMAT, which eluted from the column at approximately $44 \mathrm{~min}$ (Figure 5). In addition, WFC cultures accumulated a novel molecule with an elution time of $41 \mathrm{~min}$ and that had an $\mathrm{m} / \mathrm{z}$ value of 259.0 in ESI LC-MS analyses. The unidentified metabolite displayed a higher fluorescence at excitation and emission wavelengths of 310 $\mathrm{nm}$ and $410 \mathrm{~nm}$, respectively, than at $272 \mathrm{~nm}$ excitation and $372 \mathrm{~nm}$ emission settings (Figure 5). Ergot alkaloids with a double bond between carbons 9 and 10 of the ergot alkaloid ring system fluoresce stronger at the $310 \mathrm{~nm}$ (excitation) and $410 \mathrm{~nm}$ (emission) setting compared to their fluorescence at the $272 \mathrm{~nm} / 372 \mathrm{~nm}$ setting; conversely, ergot alkaloids with a 8,9 double bond or those lacking a double bond at either of those positions fluoresce stronger at the $272 \mathrm{~nm} / 372 \mathrm{~nm}$ setting (Panaccione et al., 2012). The novel alkaloid was not produced in sufficient quantities to allow the acquisition of additional structural data at this time.

\section{Discussion}

Our results describe the first transfer of ergot alkaloid biosynthetic capability to a previously non-producing fungus. These data also indicate that $d m a W$, easF, easE, and eas $C$ are sufficient for $A$. nidulans to synthesize chanoclavine-I. Since the pathway leading to the formation of chanoclavine-I appears to be evolutionarily conserved, the knowledge gained from this study will not only apply to $A$. fumigatus but theoretically to all ergot alkaloid producers. Despite the large amount of research conducted on ergot alkaloid synthesis, the formation of chanoclavine-I has yet to be completely understood. Only two steps in this evolutionarily conserved portion of the pathway have been 
completely characterized- those controlled by dmaW and easF (Wang et al., 2004; Rigbers and Li, 2008; Unsöld and Li, 2005; Coyle and Panaccione, 2005). After the formation of N-Me-DMAT, the order of activity and catalytic functions of enzymes is not clear. Our data indicate that EasE and EasC individually are necessary and together are sufficient to convert $N$-Me-DMAT to chanoclavine-I. Nonetheless, the observations in this study do not rule out the possibility that $A$. nidulans naturally possesses an enzyme that also contributes to the formation of chanoclavine-I from $N$-Me-DMAT. Despite the fact that all known ergot alkaloid genes are clustered in A. fumigatus and other ergot alkaloid-producing fungi (Lorenz et al., 2009; Panaccione, 2010; Schardl et al., 2013), we cannot presently exclude the possibility that a required gene in the pathway may be located elsewhere in the genome and common to nonproducing fungi.

Transformations with subsets of three of the four studied genes, combining alternately eas $\mathrm{C}$ or easE with the combination of $d m a W$ and easF, did not yield strains capable of producing chanoclavine-I. These results support previous knockout data indicating that both eas $E$ and eas $C$ are required for chanoclavine-I synthesis from N-Me-DMAT (Goetz et al., 2011). A metabolite of undetermined structure accumulated to relatively high levels in the WFC transformant; this metabolite was not detectable in extracts of the A. nidulans WFE strain. Improved strains or altered growth conditions will be necessary to increase yield of the compound for the purpose of collecting adequate material for structure determination. The WFC analyte could potentially be the next intermediate downstream of $\mathrm{N}$-MeDMAT, in which case easC would code for the enzyme involved in the third step of ergot alkaloid synthesis. Considering its catalase activity, one proposed function for Eas $\mathrm{C}$ is detoxification of hydrogen peroxide produced from the oxidative activities of EasE (Goetz et al., 2001), in which case EasC would not act directly on an ergot alkaloid substrate; however, comparison of the alkaloid profiles of WFE and WFC mutants indicates that EasC can act independent of EasE and chemically modifies an ergot alkaloid substrate. In a previous study, easE was knocked out in $A$. fumigatus, creating a strain of $A$. fumigatus that lacked easE but contained easC (Goetz et al., 2011). N-Me-DMAT was detected in cultures of the easE knockout strain, but the novel metabolite from the $A$. nidulans WFC strain was not observed. One potential explanation for this apparent discrepancy is that the $A$. fumigatus easE knockout mutant contained downstream enzymes in the ergot alkaloid pathway that may have altered its ergot alkaloid profile. Alternatively, the difference between alkaloid profiles of $A$. fumigatus eas $E$ knockout and $A$. nidulans WFC could be due to other factors in the species backgrounds in which N-Me-DMAT accumulated. It is presently unknown whether the observed WFC analyte is an actual, on-pathway intermediate or whether it is a byproduct of EasC acting out of sequence on a compound that is not 
ordinarily its substrate. Feeding studies with the unidentified analyte and strains expressing defined sets of ergot alkaloid synthesis genes would be a reasonable approach to test the involvement of this compound in the ergot alkaloid pathway. Again, greater quantities of the analyte would be required for such studies. An in vitro approach involving separate and combined enzymes also would help to fully understand the functions of these enzymes. Difficulty with expressing EasE in active form in vitro has slowed studies of catalytic function of this enzyme (Goetz et al., 2011). The approach of expressing genes in A. nidulans may be applied to other genes in the pathway to study their roles in the biosynthesis of ergot alkaloids (as was recently reviewed by Chiang et al. (2011) for other fungal secondary metabolite pathways). Heterologous expression in $A$. nidulans also should be applicable to studying genes from ergot alkaloid pathways of other fungi, such as those genes from lineage-specific branches of the pathway. In such cases, it may be necessary to replace the native promoters of those heterologous genes with promoters from $A$. fumigatus eas genes or strong $A$. nidulans promoters.

\section{References}

Chiang YM, Chang SL, Oakley BR, Wang CC. 2011. Recent advances in awakening silent biosynthetic gene clusters and linking orphan clusters to natural products in microorganisms. Curr. Opin. Chem. Biol. 15: 137-143.

Coyle CM, Panaccione DG. 2005. An ergot alkaloid biosynthesis gene and clustered hypothetical genes from Aspergillus fumigatus. Appl. Environ. Microbiol. 71: 3112-3118.

Coyle CM, Kenaley SC, Rittenour WR, Panaccione DG. 2007. Association of ergot alkaloids with conidiation in Aspergillus fumigatus. Mycologia 99: 804-811.

Coyle CM, Cheng JZ, O'Connor SE, Panaccione DG. 2010. An old yellow enzyme gene controls the branch point between Aspergillus fumigatus and Claviceps purpurea ergot alkaloid pathways. Appl. Environ. Microbiol. 76: 3898-3903.

Goetz KE, Coyle CM, Cheng JZ, O'Conner S, Panaccione DG. 2011. Ergot cluster-encoded catalase is required for synthesis of chanoclavine-I in Aspergillus fumigatus. Curr. Genet. 57: 201-211.

Hunter SH, Provasoli L, Schatz A, Haskins CP. 1950. Some approaches to the study of the role of metals in the metabolism of microorganisms. Am. Philos. Soc. Proc. 94: 152-170.

Kozikowski A, Chen C, Wu J, Shibuya M, Kim C, Floss H. 1993. Probing ergot alkaloid biosynthesis: Intermediates in the formation of ring C. J. Am. Chem. Soc. 115: 2482-2488.

Latgé J-P. 1999. Aspergillus fumigatus and aspergillosis. Clin. Microbiol. Rev. 12: 310-350.

Lorenz N, Haarmann T, Pažoutová S, Jung M, Tudzynski P. 2009. The ergot alkaloid gene cluster: Functional analyses and evolutionary aspects. Phytochem. 70: 1822-1832.

Lorenz N, Olšovská J, Šulc M, Tudzynski P. 2010. Alkaloid cluster gene ccsA of the ergot fungus Claviceps purpurea encodes chanoclavine I synthase, a flavin adenine dinucleotide-containing oxidoreductase mediating the transformation of $\mathrm{N}$-methyl-dimethylallyltryptophan to chanoclavine. Appl. Environ. Microbiol. 76: 1822-1830. 
Panaccione DG. 2010. Ergot alkaloids. The Mycota, Vol. X, Industrial Applications, 2nd Edition (Ed. M. Hofrichter), Berlin-Heidelburg: Springer-Verlag, 195-214.

Panaccione DG, Ryan KL, Schardl CL, Florea, S. 2012. Analysis and modification of ergot alkaloid profiles in fungi. Method. Enzymol. 515: 267-290.

Richards, E, Reichardt M, Rogers S. 2001. Preparation of genomic DNA from plant tissue. Curr. Protoc. Mol. Biol. 27: 2.3.1-2.3.7.

Rigbers O, Li SM. 2008. Ergot alkaloid biosynthesis in Aspergillus fumigatus overproduction and biochemical characterization of a 4-dimethylallytryptophan N-methyltransferase. J. Biol. Chem. 283: 26859-26868.

Schardl CL, Young CA, Hesse U, Amyotte SG, Andreeva K, Calie PJ, Fleetwood DJ, Haws DC, Moore N, Oeser B, Panaccione DG, Schweri KK, Voisey CR, Farman ML, Jaromczyk JW, Roe BA, O'Sullivan DM, Scott B, Tudzynski P, An Z, Arnaoudova EG, Bullock CT, Charlton ND, Chen L, Cox M, Dinkins RD, Florea S, Glenn AE, Gordon A, Güldener U, Harris DR, Hollin W, Jaromczyk J, Johnson RD, Khan AK, Leistner E, Leuchtmann A, Li C, Liu JG, Liu J, Liu M, Mace W, Machado C, Nagabhyru P, Pan J, Schmid J, Sugawara K, Steiner U, Takach JE, Tanaka E, Webb JS, Wilson EV, Wiseman $\mathrm{JL}$, Yoshida RY, Zeng Z. 2013. Plant-symbiotic fungi as chemical engineers: multi-genome analysis of the Clavicipitaceae reveals dynamics of alkaloid loci. Plos Genetics 9: e1003323.

Twumasi-Boateng K, Yu Y, Chen D, Gravelat FN, Nierman WC, Sheppard DC. 2009. Transcriptional profiling identifies a role for BrlA in the response to nitrogen depletion and for StuA in the regulation of secondary metabolite clusters in Aspergillus fumigatus. Eukaryot. Cell 8: 104-115.

Unsöld IA, Li S-M. 2005. Overproduction, purification and characterization of FgaPT2, a dimethylallyltryptophan synthase from Aspergillus fumigatus. Microbio. 151: 1499-1505.

Wallwey C, Li S-M. 2011. Ergot alkaloids: Structure diversity, biosynthetic gene clusters and functional proof of biosynthetic genes. Nat. Prod. Rep. 28: 496-510.

Wang J, Machado C, Panaccione DG, Tsai H-F, Schardl CL. 2004. The determinate step in ergot alkaloid biosynthesis by an endophyte of perennial ryegrass. Fungal Genet. Biol. 41: 189-198. 


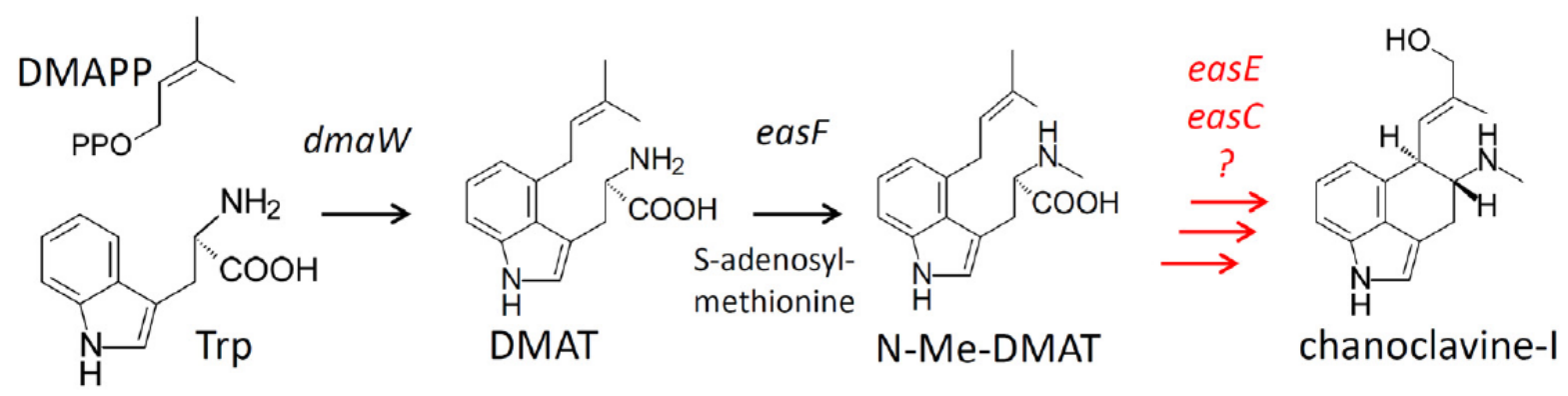

Figure 1: Early steps of the ergot alkaloid pathway. Genes controlling specific steps are listed beside arrows. Abbreviations: DMAPP = dimethylallyldiphosphate; $\operatorname{Trp}=$ tryptophan; DMAT = dimethylallyltryptophan. Unresolved steps are indicated in red. 


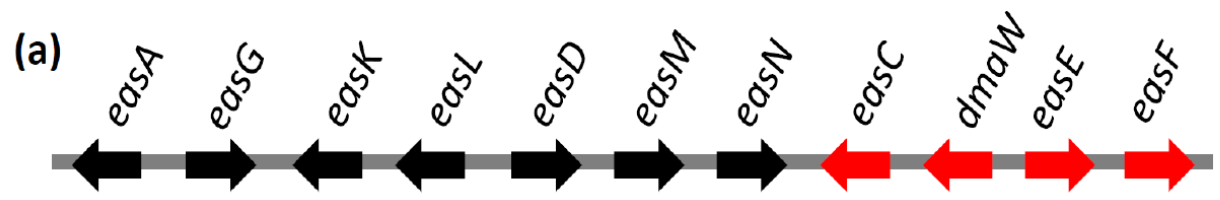

(b)

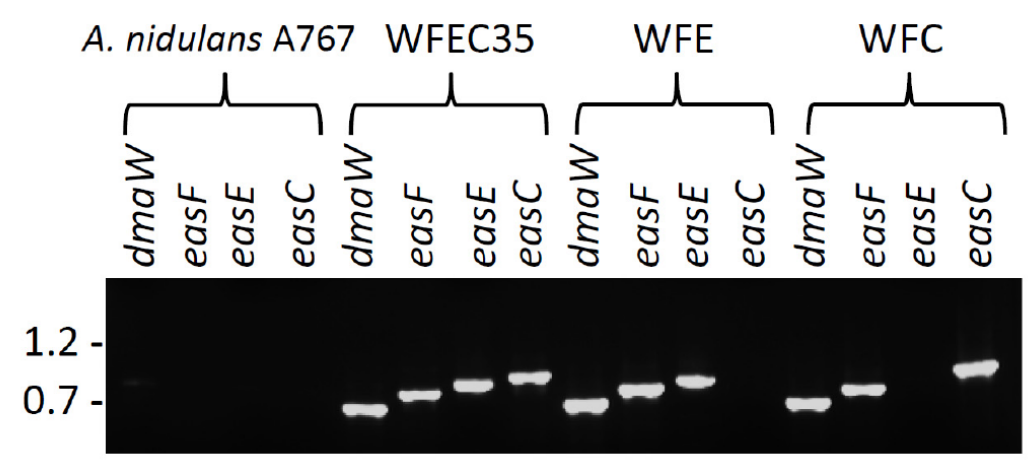

Figure 2: Ergot alkaloid synthesis genes. (a) The ergot alkaloid gene cluster of $A$. fumigatus. Genes expressed in $A$. nidulans in this study are shown in red; (b) PCR verification of the presence or absence of $d m a W$, easF, easE, and eas $C$ from wild type and mutant strains. Each gene was independently amplified from internal primer binding sites. Relative mobility of relevant BstEll-digested bacteriophage lambda fragments (sizes in $\mathrm{kb}$ ) is indicated to the left of the gel. 


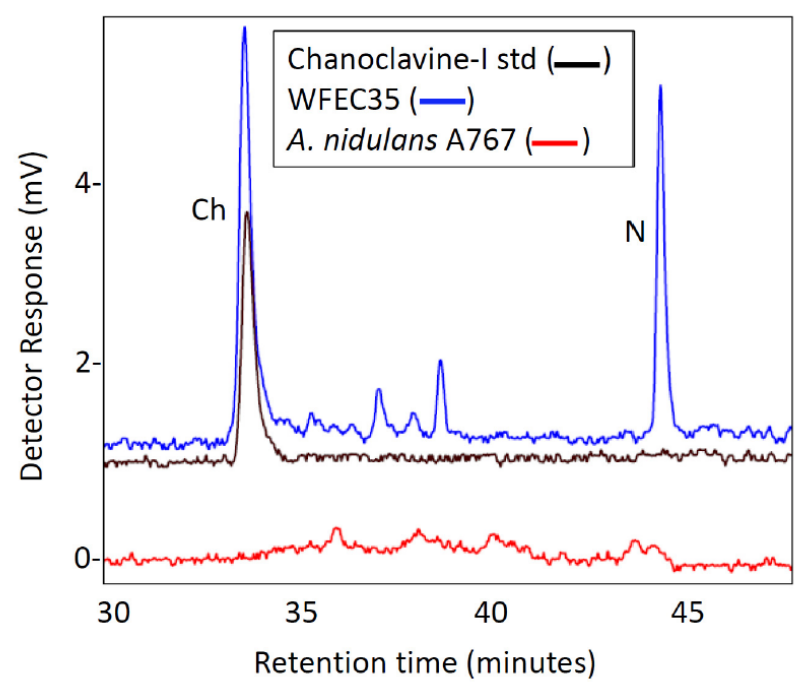

Figure 3: HPLC analyses of $A$. nidulans strain WFEC35 (transformed with dmaW, eas $F$, easE, and easC), $A$. nidulans FGSC A767, and chanoclavine-I standard. Ch, chanoclavine-I; N, N-Me-DMAT. Fluorescence properties were observed with an excitation wavelength of $272 \mathrm{~nm}$ and an emission wavelength of 372 $\mathrm{nm}$. 

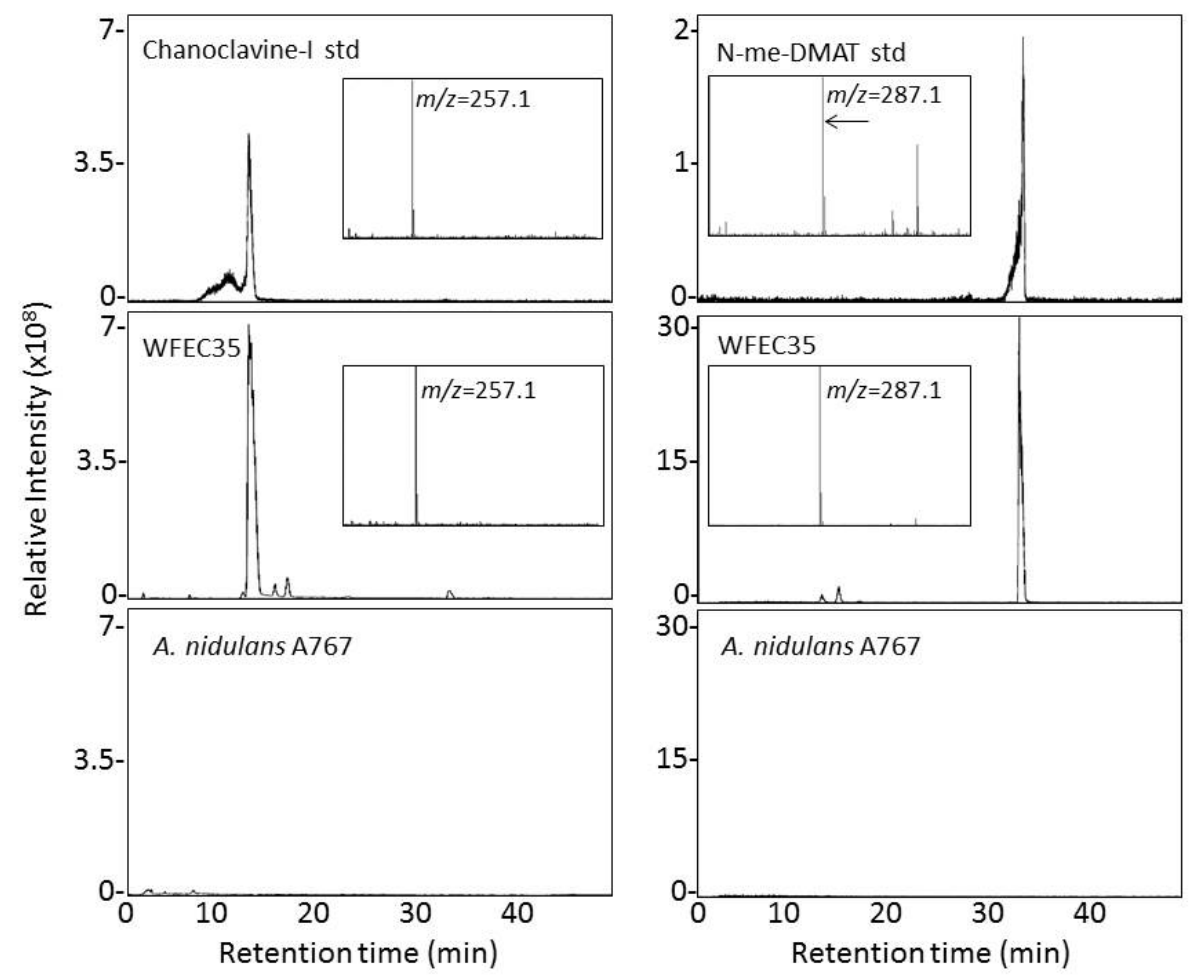

Figure 4: LC-MS analysis of $A$. nidulans extracts. Chromatograms display presence and elution time of molecules with an $m / z$ value of $257 \pm 0.5$ (left column) or $287 \pm 0.5$ (right column). Chromatograms of $A$. nidulans strains A767 and WFEC35 were normalized to the intensity of the highest peak in the WFEC35 trace for each analyte. The $N$-Me-DMAT standard chromatogram is displayed at a higher sensitivity because of paucity of material available. Inserts show the mass spectrum of the major peak in each chromatogram. 

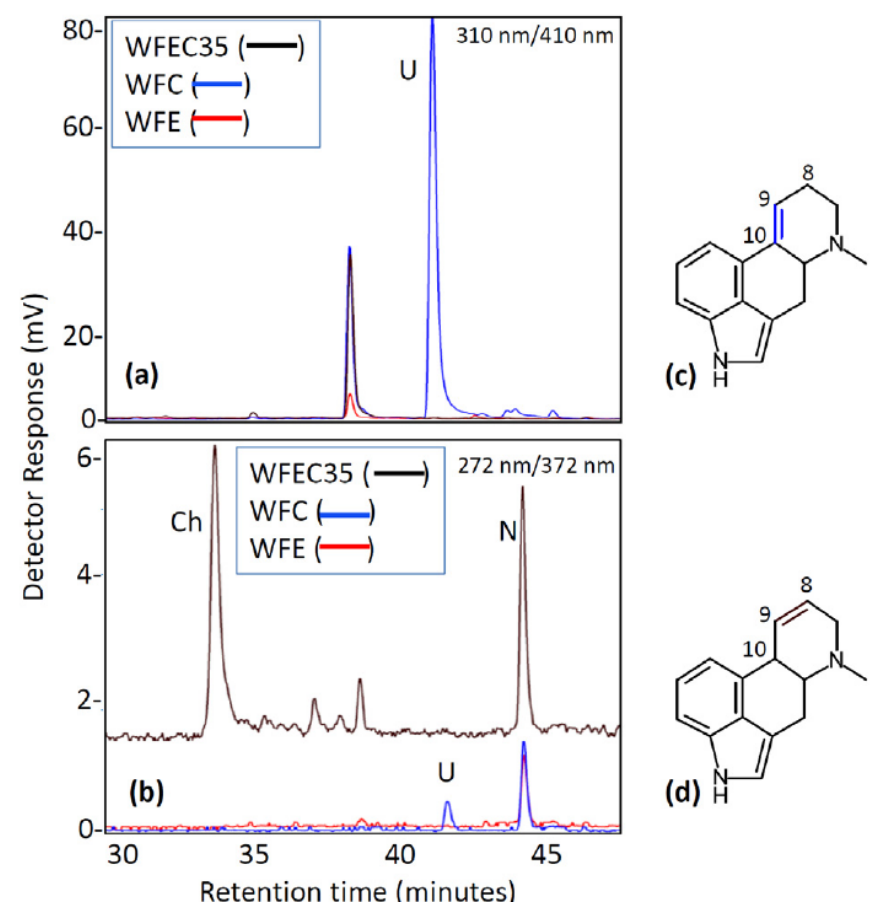

(d)

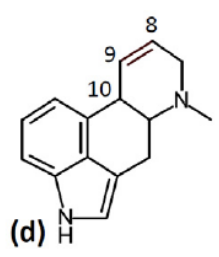

Figure 5: Fluorescence HPLC analysis of WFC and WFE mutant cultures. (a) Chromatogram with excitation at $310 \mathrm{~nm}$ and emission at $410 \mathrm{~nm}$; (b) Chromatogram with excitation at $272 \mathrm{~nm}$ and emission at $372 \mathrm{~nm}$; (c) Generic ergoline ring system with double bond between carbons 9 and 10; (d) Generic ergoline ring system with double bond between carbons 8 and 9. Ch, chanoclavine-I; N, N-Me-DMAT; U, Unknown unique to strain WFC. 


\title{
Chapter 3
}

\section{Identification and structural elucidation of a new ergot alkaloid, ergotryptamine, produced by genetically modified Aspergillus nidulans and natural variants of Epichloë}

\author{
Katy L. Ryan, Novruz G. Akhmedov and Daniel G. Panaccione \\ Published in revised form as: \\ Journal of Agricultural and Food Chemistry doi: 10.1021/jf505718x
}




\section{Abstract}

Ergot alkaloid pathway reconstruction in Aspergillus nidulans is an approach used to better understand the biosynthesis of these mycotoxins. A mutant strain named A. nidulans WFC (expressing ergot alkaloid synthesis genes $d m a W$, eas $F$, and eas $C$ ) produced the established intermediate $\mathrm{N}$ methyldimethylallyltryptophan (N-Me-DMAT), as well as an uncharacterized ergot alkaloid. We investigated the chemical structure of this new metabolite and its role in the ergot alkaloid pathway. Mass spectrometry, labeling, and NMR studies showed that the unknown ergot alkaloid, designated here as ergotryptamine, differed from N-Me-DMAT by the loss of the carboxyl group, addition of a hydroxyl group, and shift in position of a carbon-carbon double bond. Feeding studies with Aspergillus mutants did not show ergotryptamine turnover suggesting it is a pathway byproduct as opposed to an authentic intermediate. Several Epichloë species also produced this metabolite and further investigations revealed the equivalency of ergotryptamine with an ergot alkaloid provisionally described as 6,7 secolysergine.

\section{Introduction}

Ergot alkaloids are a class of indole-derived mycotoxins with agricultural and pharmaceutical significance. Several fungi in the family Clavicipitaceae (order Hypocreales) and a few fungi in the distantly related family Trichocomaceae (order Eurotiales) produce these secondary metabolites (Panaccione, 2010; Schardl et al., 2013a). The Epichloë spp. (Clavicipitaceae) are endophytes of turf and forage grasses (Poaceae, subfamily Poöideae) and synthesize a variety of compounds toxic to vertebrates and invertebrates (Schardl et al., 2013b). Ergot alkaloids in these grass symbionts cause toxicoses in grazing animals and are responsible for significant economic loss to livestock industries (Strickland et al., 2012; Thompson et al., 1993). The phylogenetically divergent fungus Aspergillus fumigatus (Trichocomaceae) is a common saprotroph and opportunistic human pathogen that causes spoilage of feed in agriculture settings (Panaccione, 2010; Latgé, 1999; Scudamore and Livesey, 1997). Compared to the Epichloë spp., A. fumigatus produces a different subset of ergot alkaloids but can serve as a prominent model organism for studying ergot alkaloid biosynthesis (Goetz et al., 2011; Unsöld and Li, 2005; Coyle et al., 2005).

There is considerable diversity in the later steps of the ergot alkaloid pathway due to genetic variation within ergot alkaloid gene clusters of different fungi; however, there is strong evidence that the early steps of the pathway are conserved leading to the formation of intermediate chanoclavine-I (Figure 1) (Panaccione, 2010; Schardl et al., 2013a). It was recently discovered that four genes, dmaW, 
eas $F$, eas $C$, and eas $E$, amplified from the $A$. fumigatus genome are sufficient to direct chanoclavine-I synthesis when transgenically expressed in Aspergillus nidulans, a model fungus that does not contain ergot alkaloid synthesizing genes or produce ergot alkaloids (Ryan et al., 2013). DmaW performs the determinate step of ergot alkaloid synthesis by prenylating tryptophan to produce dimethylallyltryptophan (DMAT) (Unsöld and Li, 2005; Coyle et al., 2005; Wang et al., 2004). The easF gene encodes a methyltransferase responsible for converting DMAT into N-Me-DMAT (Rigbers and Li, 2008). The exact reactions catalyzed by EasE and EasC have not yet been determined, but previous work demonstrates that these two genes are required to convert N-Me-DMAT to chanoclavine-I (Goetz et al., 2011; Ryan et al., 2013; Lorenz et al., 2010). Bioinformatics suggest that EasC is a catalase, whereas EasE is an oxidoreductase. A scheme of conversion from N-Me-DMAT to chanoclavine-I has been proposed by Kozikowski et al. (1993) (Figure 1).

Previously Ryan et al. (2013) investigated the roles of EasC and EasE, as well as the intermediates they produced, by reconstructing the early steps of ergot alkaloid synthesis in $A$. nidulans. A mutant strain named $A$. nidulans WFC (containing the genes $d m a W$, easF, and eas $C$ from $A$. fumigatus) accumulated ergot alkaloid pathway intermediate N-Me-DMAT as well as an uncharacterized ergot alkaloid (defined here as alkaloid X) not seen in control strains and transformant $A$. nidulans WFE (expressing dmaW, easF and easE), which synthesized N-Me-DMAT but no unusual metabolites. Alkaloid X was detected by HPLC with fluorescence detection. Consistent with ergot alkaloids with a double bond conjugated to the indole ring (Panaccione et al., 2012), this compound displayed an intense peak when detected with a fluorescence setting of $310 \mathrm{~nm}$ excitation and $410 \mathrm{~nm}$ emissions, while a much weaker response was detected at $272 \mathrm{~nm}$ excitation and $372 \mathrm{~nm}$ emissions. ESI LC/MS analyses determined alkaloid $X$ to have a $m / z$ value of 259.0. Based on the genetic background of the modified $A$. nidulans strain that produces this compound and its chromatographic and fluorescence properties, the uncharacterized metabolite was proposed to be a derivative of N-Me-DMAT. In this study, we investigated the chemical structure of alkaloid X, its role as a pathway intermediate or byproduct, and determined whether natural ergot alkaloid producers accumulated this metabolite.

\section{Methods}

Large scale extraction and isolation of ergot alkaloids: $A$. nidulans WFC transformants were previously generated by Ryan et al. (2013). Cultures were grown on modified ST medium ( $20 \mathrm{~g} / \mathrm{L}$ sucrose, $20 \mathrm{~g} / \mathrm{L}$ tryptone, $1 \mathrm{~g} / \mathrm{L} \mathrm{MgSO}_{4}, 2 \mathrm{~mL} / \mathrm{L}$ trace element solution (Hunter et al., 1950), $50 \mathrm{mg} / \mathrm{L}$ nicotinic acid, $1 \mathrm{~g} / \mathrm{L}$ tryptophan, $20 \mathrm{~mL} / \mathrm{L}$ corn steep liquor, $15 \mathrm{~g} / \mathrm{L}$ agar) for seven days at $37^{\circ} \mathrm{C}$. Ergot alkaloids were 
extracted by soaking fungal mats in $10 \%$ aqueous ammonium carbonate ( $25 \mathrm{~mL}$ solvent per one $100 \times 15$ $\mathrm{mm}$ petri dish) for two hours followed by centrifugation at 5000 RPM for 10 minutes to pellet spores and mycelium. The extract was purified over an Isolute ${ }^{\circledR}$ C18 SPE column (500 mg/3 mL; Biotage, Charlotte, NC, USA). The column was pre-rinsed with $2 \mathrm{~mL}$ methanol followed by $2 \mathrm{~mL}$ deionized, distilled water. Ergot alkaloid extracts were applied to the column allowing a flow rate of approximately $1 \mathrm{~mL} / \mathrm{min}$. The column was then washed with $3 \mathrm{~mL}$ water followed by $3 \mathrm{~mL}$ methanol to remove most ergot alkaloids except alkaloid $\mathrm{X}$ which required addition of acid for elution. Alkaloid $\mathrm{X}$ was eluted in 3 $\mathrm{mL} 98 \%$ methanol + 2\% (v/v) glacial acetic acid (for NMR spectroscopy described below) or 99.95\% methanol $+0.05 \%(v / v)$ glacial acetic acid (for Aspergillus feeding studies described below). For NMR analyses, alkaloid X was HPLC purified (described below) and the fraction was reapplied to an Isolute ${ }^{\circledR}$ C18 column following the procedure described above and, after elution, concentrated in a vacuum centrifuge until all solvent had evaporated. For Aspergillus feeding studies, eluate was concentrated to a final volume of $10 \mu \mathrm{L}$. N-Me-DMAT and the $\mathrm{m} / \mathrm{z} 303.0$ metabolite were HPLC purified from the $100 \%$ methanol SPE column eluate and concentrated to dryness.

Labeled tryptophan feeding studies with $A$. nidulans WFC: Ten mg of L-tryptophan- $d_{5}$ (indole $d_{5}$ ) or Ltryptophan- $1-{ }^{13} \mathrm{C}$ (both from Sigma-Aldrich, St. Louis, MO, USA) was dissolved in one $\mathrm{mL}$ water, filter sterilized through a $0.22-\mu \mathrm{m}$ filter, and added to $99 \mathrm{~mL}$ of SYE agar $(20 \mathrm{~g} / \mathrm{L}$ sucrose, $20 \mathrm{~g} / \mathrm{L}$ yeast extract, $1 \mathrm{~g} / \mathrm{L} \mathrm{MgSO}$, $50 \mathrm{mg} / \mathrm{L}$ nicotinic acid, $2 \mathrm{~mL} / \mathrm{L}$ trace element solution) after autoclaving but prior to solidification of the medium. This medium was inoculated with $A$. nidulans WFC and incubated for one week at $37^{\circ} \mathrm{C}$. Ergot alkaloids were extracted by repeatedly washing the fungal culture surface with 2 $\mathrm{mL}$ of methanol. The suspended cultures were incubated in methanol for 30 minutes followed by centrifugation to remove fungal matter. Extracts were concentrated in a vacuum centrifuge to $50 \mu \mathrm{L}$ for ESI MS/LC analysis.

Feeding study with Aspergillus mutants and the alkaloid X: A. nidulans WFE was described in Ryan et al. (2013) and $A$. fumigatus easC and easE knockout mutants were previously generated by Goetz et al. (2011). A total of $1 \times 10^{6}$ spores were inoculated in $200 \mu \mathrm{L}$ of liquid sucrose malt medium $(15 \mathrm{~g} / \mathrm{L}$ malt extract broth and $20 \mathrm{~g} / \mathrm{L}$ sucrose) supplemented with $50 \mu \mathrm{mol}$ of alkaloid $\mathrm{X}$ and incubated for five days at $37^{\circ} \mathrm{C}$. Ergot alkaloids were extracted by the addition of $200 \mu \mathrm{L}$ methanol, vortexing briefly, and incubating for 30 minutes at room temperature followed by centrifugation for solution clarification. Samples were then analyzed by HPLC (as described below).

Yield of alkaloid X: A. nidulans WFC transformants were grown on ST agar (as described above) for 10 days at $37^{\circ} \mathrm{C}$. One square $\mathrm{cm}$, surface area, of fungal culture was incubated in $400 \mu \mathrm{L}$ methanol for 30 
minutes. Samples were centrifuged to remove fungal matter followed by HPLC analysis. Metabolite yield was calculated by comparison of peak areas to an ergonovine (Sigma, St. Louis, MO) standard curve. The number of spores in suspension were counted on a hemacytometer (Hausser Scientific, Horsham, PA).

Extraction of ergot alkaloids from Epichloë sp.-infected grasses: An IpsA knockout strain Epichloë festucae var. Iolii x E. typhina isolate Lp1 (henceforth E. sp. Lp1) was previously generated and inoculated into perennial ryegrass (Lolium perenne) (Panaccione et al., 2001). Other endophyte infected grasses were provided by Christopher Schardl (University of Kentucky). The Epichloë spp. studied included several species recently realigned from the genus Neotyphodium and have previously been known by their Neotyphodium sp. names. Ergot alkaloids were extracted from fungal-infected grass pseudostems as previously described (Panaccione et al., 2012).

High Performance Liquid Chromatography analysis: Samples were loaded onto a C18 column (Prodigy $5-\mu \mathrm{m}$ ODS3 (150 mm by $4.6 \mathrm{~mm}$ ); Phenomenex, Torrance, CA, USA) and subjected to a gradient of mobile phases $A(5 \% \mathrm{v} / \mathrm{v}$ acetonitrile $+95 \% \mathrm{v} / \mathrm{v} 50 \mathrm{mM}$ ammonium acetate) and B (75\% acetonitrile + 25\% $50 \mathrm{mM}$ ammonium acetate) as previously described by Panaccione et al. (2012). Ergot alkaloids were detected by their fluorescence at two detector settings: $272 \mathrm{~nm}$ excitation and $372 \mathrm{~nm}$ emission, and $310 \mathrm{~nm}$ excitation and $410 \mathrm{~nm}$ emission. For isolation of metabolites, HPLC runoff was collected for 30 seconds after metabolite of interest was visualized by the detectors.

Electrospray Ionization Mass Spectrometry/Liquid Chromatography analysis: For most analyses (other than comparison of alkaloid X from A. nidulans WFC to compounds extracted from Epichloë sp.-infected grasses), $10 \mu \mathrm{L}$ of concentrated fungal extract was injected into a Finnigan LCQ DecaXP plus mass spectrometer equipped with a Surveyor HPLC system. Analytes were separated on a C18 column (Phenomenex $4-\mu \mathrm{m}$ polar-RP $(150 \mathrm{~mm}$ by $2 \mathrm{~mm})$ ) maintained at $30^{\circ} \mathrm{C}$, by combining mobile phases $\mathrm{A}$ ( $5 \%$ acetonitrile, $0.1 \%$ formic acid) and B (75\% acetonitrile, $0.1 \%$ formic acid) as described by Ryan et al. (2013). The flow rate was $200 \mu \mathrm{L} / \mathrm{min}$. Analytes were ionized by electrospray ionization in positive mode and were detected by scanning for ions with a $m / z$ between 200-400. $\mathrm{MS}^{2}$ scans for compounds of $m / z=259.0$ also were conducted. For isotope incorporation, $m / z$ intensities were recorded at peak retention time (alkaloid $X$ retention time $=22 \mathrm{~min}$; N-me-DMAT retention time $=33 \mathrm{~min}$ ) for the following peaks $m / z 259.0$ [alkaloid $X+\mathrm{H}^{+}, m / z 260.0$ [alkaloid $\mathrm{X}+1+\mathrm{H}$ ] $^{+}, \mathrm{m} / \mathrm{z} 263.0$ [alkaloid $\mathrm{X}+4+$ $\mathrm{H}^{+}, m / z 287.0[\mathrm{~N}-\mathrm{me}-\mathrm{DMAT}+\mathrm{H}]^{+}, m / z 288.0[\mathrm{~N}-\mathrm{me}-\mathrm{DMAT}+1+\mathrm{H}]^{+}$, and $\mathrm{m} / z 291.0$ [N-me-DMAT $+4+$ $\mathrm{H}]^{+}$. Eight intensity readings for each metabolite were recorded around peak intensity. Student's t-test 
was performed with JMP (SAS, Cary, NC) to compare sample means of $m / z$ intensity ratios for alkaloid X and $\mathbf{4}$ between samples grown on labeled $\mathbf{2}$ supplemented medium and unsupplemented medium.

Analyses of alkaloid X from $A$. nidulans WFC in comparison to compounds extracted from Epichloë sp.-infected grasses were conducted with different instrumentation and methods. Twenty $\mu \mathrm{L}$ of concentrated sample was analyzed on a ThermoScientific $Q$ Exactive mass spectrometer. Analytes were separated on a Thermo Scientific Hypersil GOLD HPLC column (1.9 $\mu \mathrm{m}$ particle size, end-capped, ultrapure, silica-based; $50 \times 2.1 \mathrm{~mm}$ ) maintained at $30^{\circ} \mathrm{C}$, by combining mobile phases $\mathrm{A}(5 \%$ acetonitrile, $0.1 \%$ formic acid) and B (75\% acetonitrile, $0.1 \%$ formic acid) over a 10 min linear gradient of $0 \% \mathrm{~B}$ to $100 \% \mathrm{~B}$. The flow rate was $300 \mu \mathrm{L} / \mathrm{min}$. Analytes were ionized by electrospray ionization in positive mode and were detected by scanning for ions with $m / z$ between $200-400$.

NMR measurements: The 1D and 2D NMR spectra were recorded using a Varian INOVA $600 \mathrm{MHz}$ spectrometer at $25^{\circ} \mathrm{C}$ operating at $599.67 \mathrm{MHz}$ and $150.79 \mathrm{MHz}$ for proton and carbon, respectively, and equipped with a triple-resonance z-axis pulsed field gradient $5 \mathrm{~mm}$ probe. Sample concentration was $0.2 \mathrm{mg} / 0.7 \mathrm{~mL}(0.001 \mathrm{M})$. The FIDs of the one and two dimensional NMR spectra were processed using the commercially available NMR software package ACD/SpecManager (Advanced Chemistry Development, Inc; Product Version 12, 2008; http:/(www.acdlabs.com). ${ }^{1} \mathrm{H}$ NMR chemical shifts are reported relative to internal standard peak (TMS- tetramethylsilane) at $0 \mathrm{ppm} .{ }^{13} \mathrm{C}$ NMR chemical shifts are reported in DMSO- $d_{6}$ relative to the central peak of the septet set to $39.5 \mathrm{ppm}$. The typical parameters for acquiring the ${ }^{1} \mathrm{H}$ NMR spectra were as follows: spectral widths $7807.16 \mathrm{~Hz}$, acquisition time $4.0 \mathrm{~s}$, pulse width $3.16 \mu \mathrm{s}\left(45^{\circ}\right)$, relaxation time $2 \mathrm{~s}$, and number of transients 128 . The parameters for the gradient selected 1D TOCSY were applied using TOCSY1D program with spectral width of 7807.16 $\mathrm{Hz}$, acquisition time of $4.0 \mathrm{~s}$, pulse width $9.5 \mu \mathrm{s}\left(90^{\circ}\right)$, relaxation time of $1 \mathrm{~s}$, mixing time was set to 80 $\mathrm{ms}$, and the number of transients used was 128 . The FIDs were processed with a line broadening function of $1 \mathrm{~Hz}(\mathrm{lb})$. The gradient selected gCOSY spectra were acquired over both F2 and F1 with a spectral width of $7807.16 \mathrm{~Hz}$, pulse width $9.0 \mu \mathrm{s}\left(90^{\circ}\right)$, relaxation delay $1 \mathrm{~s}$, and 512 time increments of 64 transients each in magnitude mode. The FID's were zero filled to give a $4 \mathrm{~K} \times 4 \mathrm{~K}$ data matrix and a sine-bell function was applied in both dimensions (F1 and F2) prior to Fourier transformations giving a digital resolution of $3.81 \mathrm{~Hz}$. Adiabatic version of gradient selected gHSQCAD and gHMBCAD spectra were acquired in phase sensitive mode with a spectral width of $7807.16 \mathrm{KHz}$ for ${ }^{1} \mathrm{H}$ (F2 dimension), a spectral width of $37700 \mathrm{~Hz}$ for ${ }^{13} \mathrm{C}$ (F1 dimension) by using a $2048 \times 512$ data matrix size and 128 transients for each $\mathrm{t} 1$ value. The carbon decoupler $90^{\circ}$ pulse width was $10.4 \mu \mathrm{s}$, one-bond coupling j1xh was set to $145 \mathrm{~Hz}$. For evolution of long-range correlation, the multiple bond $\mathrm{CH}$ coupling constant jnxh 
was set to $8 \mathrm{~Hz}$ and relaxation delay was set to $1.5 \mathrm{~s}$. The FIDs of proton detected gHSQCAD were processed with a Gaussian weighting function in both dimensions, whereas the FID's of proton detected gHMBCAD were processed with a sine bell weighting function in F2 and with a Gaussian weighting function in F1 (carbon dimension).

The NMR parameters (chemical shifts and coupling constants) for alkaloid $\mathrm{X}$ were determined by iterative analysis using the gNMR program. As starting data the iterative process used ${ }^{1} \mathrm{H}$ NMR chemical shifts and coupling constants estimated from the experimental spectra.

\section{Results and Discussion}

\section{Insights on the structure of alkaloid $X$.}

Aspergillus nidulans WFC cultures grown on ST medium or ST medium supplemented with indole $d_{5}$ labeled tryptophan were analyzed by ESI LC/MS for the incorporation of deuterated tryptophan into alkaloid $\mathrm{X}$ as well as known ergot alkaloid intermediate $\mathrm{N}$-me-DMAT. During the first step of ergot alkaloid synthesis, DmaW replaces a deuterium on indole-carbon 5 with a prenyl group resulting in accumulation of ions with $m / z$ values consistent with $[\mathrm{M}+4+\mathrm{H}]^{+}$rather than $[\mathrm{M}+5+\mathrm{H}]^{+}$. Fungal cultures grown on medium supplemented with $d_{5}$ labeled tryptophan contained a significantly higher ratio of intensity values of $m / z 291.0$ [N-me-DMAT $+4+\mathrm{H}]^{+}$to $m / z 287.0[\mathrm{~N}-\mathrm{me}-\mathrm{DMAT}+\mathrm{H}]^{+}$ compared to control cultures grown on medium lacking $d_{5}$ labeled tryptophan (Figure 2). Similarly, extracts from fungi grown on medium supplemented with $d_{5}$ labeled tryptophan had a significantly higher ratio of intensity values of $\mathrm{m} / \mathrm{z} 263.0$ [alkaloid $\mathrm{X}+4+\mathrm{H}$ ] ${ }^{+}$to $\mathrm{m} / \mathrm{z} 259.0$ [alkaloid $\left.\mathrm{X}+\mathrm{H}\right]^{+}$, compared to controls (Figure 2). In conjunction with genetic data that demonstrated accumulation of alkaloid $\mathrm{X}$ is dependent on expression of three ergot alkaloid biosynthesis genes, the incorporation of deuterated tryptophan into alkaloid $\mathrm{X}$ confirms the new alkaloid is a derivative of tryptophan, and the accretion of $m / z$ values consistent with $[\mathrm{M}+4+\mathrm{H}]^{+}$rather than $[\mathrm{M}+5+\mathrm{H}]^{+}$, similar to what was observed with $\mathrm{N}$ me-DMAT, supports its membership in the ergot alkaloid family.

Based on high-resolution ESI LC/MS analyses, alkaloid $X$ has a molecular formula of $\mathrm{C}_{16} \mathrm{H}_{22} \mathrm{~N}_{2} \mathrm{O}$. When considered in relation to $\mathrm{N}$-me-DMAT, the elemental formula of alkaloid $\mathrm{X}$ is consistent with a loss of the carboxyl group and addition of an oxygen. The absence of the carboxyl group from alkaloid X was tested by culturing $A$. nidulans WFC on medium supplemented with L-tryptophan- $1-{ }^{13} \mathrm{C}$. The presence of $\mathrm{N}$-me-DMAT in these cultures provided a positive control for the incorporation of L-tryptophan-1-13 C. ESI LC/MS analyses of extracts from these cultures showed a significantly greater ratio of intensity values of $m / z 288.0$ [N-me-DMAT $+1+\mathrm{H}]^{+}$to $\mathrm{m} / \mathrm{z} 287.0$ [N-me-DMAT $\left.+\mathrm{H}\right]^{+}$compared to extracts from 
non-supplemented control cultures (Figure 3), consistent with presence of the tryptophan-derived carboxyl group in N-me-DMAT. However, there was no difference in the ratio of intensity values of $m / z$ 260.0 [alkaloid $\mathrm{X}+1+\mathrm{H}]^{+}$to $\mathrm{m} / \mathrm{z} 259.0$ [alkaloid $\left.\mathrm{X}+\mathrm{H}\right]^{+}$in cultures grown on supplemented medium compared to controls (Figure 3). The lack of increased ${ }^{13} \mathrm{C}$ isotope incorporated into alkaloid $\mathrm{X}$ demonstrates the absence of the carboxyl group derived from tryptophan in the chemical structure.

Considering genetic data on the origin of alkaloid X (Ryan et al., 2013) along with mass spectral, labeling, and fluorescence data, certain aspects of the structure of the compound can be proposed. The compound appears to be a derivative of N-me-DMAT, with a double bond conjugated to the indole ring, and lacking the carboxyl group. The compound also has an additional hydroxyl. We hypothesized that the hydroxyl was located on the dimethylallyl-derived carbon 16 (customarily called carbon 8 in tri- and tetracyclic ergot alkaloids) as seen in Figure 4a. Oxidation of N-me-DMAT at this position would result in the double bonding shifting from the 15,16 position to the 14,15 position (conjugated to the indole ring), accounting for the fluorescence properties of the molecule.

\section{Structural elucidation alkaloid $X$ by NMR techniques.}

The 1D and 2D NMR techniques including ${ }^{1} \mathrm{H}, 1 \mathrm{D}$ TOCSY, gradient selected ${ }^{1} \mathrm{H}-{ }^{1} \mathrm{H}$ gCOSY, and adiabatic versions of proton detected gHSQCAD and gHMBCAD experiments were used to investigate the chemical structure of alkaloid $X$, more specifically to identify the positions of the hydroxyl group and carbon double bond. In order to support the structural integrity of alkaloid X, ${ }^{1} \mathrm{H}$ and ${ }^{13} \mathrm{C} \mathrm{NMR}$ spectra of tryptophan acquired in DMSO- $d_{6}$ were accomplished with the aid of 2D NMR techniques including gCOSY, gHSQCAD and gHMBCAD. The lack of downfield proton H5 ( $\delta 7.57)$ of the indole moiety of tryptophan confirms $\mathrm{H} 5$ substitution by the prenyl group (Figure 5). The ${ }^{13} \mathrm{C} N \mathrm{NR}$ spectrum of alkaloid $\mathrm{X}$ was not acquired due to insufficient quantities (concentration $0.001 \mathrm{M}$ ). A. nidulans WFC cultures produced a low concentration of alkaloid $X$ with an average yield of $50 \mathrm{ng} / \mathrm{cm}^{2}$ surface area of sporulating culture and average spore yield of $58 \mathrm{fg} / \mathrm{spore}$.

The ${ }^{1} \mathrm{H}$ NMR spectrum (Figure 5 ) of alkaloid $\mathrm{X}$ in DMSO- $d_{6}$ exhibited four distinct downfield aromatic protons of the indole moiety at $\delta 7.10$ (doublet, $J=2.4 \mathrm{~Hz}$ ), $\delta 7.20$ (doublet of doublets, $J=7.5$, $0.8 \mathrm{~Hz}$ ), $\delta 6.99$ (doublet of doublets, $J=7.9,7.5 \mathrm{~Hz}$ ), and $\delta 7.01$ (doublet of doublets, $J=7.9,0.8 \mathrm{~Hz}$ ) assigned as $\mathrm{H} 2, \mathrm{H} 6, \mathrm{H} 7$, and $\mathrm{H} 8$, respectively. Proton-proton connectivities $(\mathrm{H} 6-\mathrm{H} 7-\mathrm{H} 8)$ through three bonds deduced from gCOSY spectrum was supported by 1D TOCSY experimental subspectra (Figure 6). Similarly, the contour plot of the gCOSY spectrum revealed cross peaks between the doublets integrating for one proton each at $\delta 6.20$ and $\delta 7.17$ with a coupling constant of ${ }^{3} \mathrm{t}_{\text {trans }}=15.8 \mathrm{~Hz}$ which were assigned to $\mathrm{H} 14$ and $\mathrm{H} 15$, respectively. Two upfield singlets integrating for six and three protons at 
$\delta 1.31$ and $\delta 2.33$, respectively were assigned to $\mathrm{H} 13, \mathrm{H} 17$ and $\mathrm{H} 18$. In the ${ }^{1} \mathrm{H}$ NMR spectrum two aliphatic methylene $\left(\mathrm{CH}_{2}\right)$ protons appeared at $\delta 2.77$ (triplet, $J=7.5 \mathrm{~Hz}$ ) and $\delta 2.98$ (triplet, $J=7.5 \mathrm{~Hz}$ ). The chemical shift assignments of these methylene protons $\left(10-\mathrm{CH}_{2}\right.$ and $\left.11-\mathrm{CH}_{2}\right)$ were based on the observed two and three bond gHMBCAD correlations. The methylene protons at $\delta 2.98$ showed longrange correlations to the carbon resonances $\mathrm{C} 2, \mathrm{C} 3$, and $\mathrm{C} 4$ of the indole moiety at $\delta 123.75, \delta 113.80$, and $\delta 124.24$, respectively, which was immediately assigned to the $10-\mathrm{CH}_{2}$. Due to unresolved coupling between $16-\mathrm{CH}_{3}$ and $\mathrm{H} 15$, the doublet lines at $\delta 7.17$ were broadened which was assigned to $\mathrm{H} 15$. Similarly, the doublet of doublets at $\delta 7.01$ is broadened due to unresolved small couplings $\left({ }^{5} J_{H H}\right)$ and was assigned to $\mathrm{H} 8$. Unambiguous assignments of $\mathrm{H} 6$ and $\mathrm{H} 8$ can be also accomplished using DPFGSENOE spectra. Since the concentration of alkaloid X was very low [0.001M (or $0.2 \mathrm{mg} / 0.7 \mathrm{~mL}$ )] this experiment was not performed. $\mathrm{NH}$ (position 12) and $\mathrm{OH}$ protons are exchangeable with water in solution and consequently did not appear in the ${ }^{1} \mathrm{H}$ NMR spectrum. However, the NH (position 1) proton of the indole ring showed broad signet at $\delta 10.80$. The assigned NMR spectral parameters $\left({ }^{1} \mathrm{H}\right.$ and ${ }^{13} \mathrm{C}$ chemical shifts $(\delta / \mathrm{ppm})$ and coupling constants $(\mathrm{J} / \mathrm{Hz})$ are listed in Table 1.

Utilization of gradient selected 1D TOCSY experiment permitted a sequential assignment of the spin systems for the different structural segments $\left[\left(C_{10}-C_{11}\right),\left(C_{6}-C_{7}-C_{8}\right)\right.$, and $\left.\left(C_{14}-C_{15}\right)\right]$ of alkaloid $X$ by means of bond connectivities. The use of the 1D TOCSY technique removed all undesired proton frequencies and gave a clean, selective excitation ${ }^{1} \mathrm{H}$ NMR subspectrum (Figure 6). All selective excitation subspectra showed only those hydrogens which are in the same ${ }^{1} \mathrm{H}$ coupling network.

Once all proton connectivities were established, an iterative spectral procedure was used to extract precise values of coupling constants for resonances of each proton (Figure 5b-d). The determined values of couplings derived from the experimental ${ }^{1} \mathrm{H}$ NMR spectrum were used as starting couplings for an iteration of multiplicity patterns for each proton peak, generating the accurate values of coupling constants. A good match between calculated and experimental splitting patterns of these protons confirmed their chemical shift assignments are correct.

A gradient version of proton-detected gHSQCAD spectrum was recorded to identify protonbearing carbons $\left(2-\mathrm{CH}, 6-\mathrm{CH}, 7-\mathrm{CH}, 8-\mathrm{CH}, 10-\mathrm{CH}_{2}, 11-\mathrm{CH}_{2}, 13-\mathrm{CH}_{3}, 14-\mathrm{CH}, 15-\mathrm{CH}, 17-\mathrm{CH}_{3}\right.$, and 18- $\left.\mathrm{CH}_{3}\right)$ on the basis of one bond correlations. The assignment of five quaternary carbons $(\mathrm{C} 3, \mathrm{C} 4, \mathrm{C} 5, \mathrm{C} 9, \mathrm{C} 16)$ was extracted on the basis of two $\left({ }^{2} J_{\mathrm{H}-\mathrm{C}}\right)$ or three $\left({ }^{3} J_{\mathrm{H}-\mathrm{C}}\right)$ gHMBCAD correlations. The contour plot of the gHMBCAD spectrum revealed a two bond correlation between $16-\mathrm{CH}_{3}$ at $\delta 1.31$ and carbon peak at $\delta$ 69.62 which is a typical chemical shift for tertiary carbon atom connected to $\mathrm{OH}$ group. 
To conclude, the analysis of the 1D and 2D NMR spectra confirm that the suggested structure of the isolated compound shown in Figure 4 a is correct. This unique ergot alkaloid produced by $A$. nidulans WFC, (3E)-2-methyl-4-\{3-[2-(methylamino)ethyl]-1H-indol-4-yl\}but-3-en-2-ol, was given the trivial name ergotryptamine due to its structural similarity to tryptamine.

\section{Role of ergotryptamine (alkaloid X) in the ergot alkaloid pathway.}

Ergotryptamine is a new member of the ergot alkaloid family. Genetic data indicate that ergotryptamine is derived from N-Me-DMAT (Ryan et al., 2013). Structural data reveal that ergotryptamine is a bicyclic ergot alkaloid lacking the carboxyl group found in precursor N-Me-DMAT (Figure 4a). The biochemical scheme proposed for chanoclavine-l synthesis (Figure 1) suggests that ergot alkaloid decarboxylation coincides with closure of the third ergoline ring (Kozikowski et al., 1993). Here we show that decarboxylation can occur without ring closure revealing that under some conditions, these events may occur via independent reactions. Through comparison of ergot alkaloid profiles of $A$. nidulans WFE and $A$. nidulans WFC (which differ only in the accumulation of ergotryptamine exclusively in A. nidulans WFC), we propose that decarboxylation of N-Me-DMAT or its oxidized derivative requires EasC. Enzymatic studies need to be performed to test the hypothesis that EasC can act as a decarboxylase.

Ergot alkaloid extracts of both $A$. nidulans WFE and A. nidulans WFC contained a molecule that was more polar than N-Me-DMAT, based on elution from a C18 column in reverse-phase HPLC (Figure 5), fluoresced strongly with $310 \mathrm{~nm}$ excitation and $410 \mathrm{~nm}$ emission settings (indicating a double bond conjugated to the indole ring), and produced a molecular ion of $m / z 303.0 \mathrm{in}$ ESI LC/MS analyses. Considering the established structures of N-Me-DMAT and ergotryptamine, we hypothesize that the $\mathrm{m} / \mathrm{z}$ 303.0 ion is N-Me-DMAT oxidized at the dimethylated carbon customarily referred to as carbon 8 . Such a proposed metabolite was previously investigated by Kozikowski et al. (1993) (Figure 4b). The later pathway ergot alkaloids agroclavine and elymoclavine are oxidized at this same carbon by peroxidases found in A. fumigatus and dozens of other fungi and plants (Scigelova et al., 1995; Panaccione et al., 2003; Coyle et al., 2010). Moreover, incubation of isolated N-Me-DMAT with our liquid modified ST medium resulted in $15 \%$ conversion to the $\mathrm{m} / \mathrm{z} 303.0$ compound suggesting N-Me-DMAT can be spontaneously oxidized, likely from the metals found in Hunter's solution.

The role of ergotryptamine as a pathway intermediate or byproduct was tested by feeding alkaloid X to Aspergillus mutants followed by an HPLC analysis for the detection of downstream pathway products. A. nidulans WFE transformants do not express eas $C$ and therefore do not synthesize ergotryptamine; however, mutant strains express easE which contains a berberine bridge domain 
expected to act on an unknown intermediate to close the third ergoline ring forming chanoclavine-I. Conversion of ergotryptamine to chanoclavine-I was not visualized in ergotryptamine fed $A$. nidulans WFE transformants. We are uncertain if this result is due to lack of EasE substrate specificity for ergotryptamine or whether $A$. nidulans potentially lacks capabilities to transport and localize ergot alkaloids for enzyme activity. Further testing was performed with $A$. fumigatus eas $C$ knockout (ko) and A. fumigatus easE ko strains. Loss of functional EasC or EasE in A. fumigatus results in a buildup of intermediate N-Me-DMAT and loss of chanoclavine-I production (Goetz et al., 2011). The expression of downstream pathway eas genes in these knockouts was previously demonstrated by chanoclavine-I feeding studies in which both knockout strains were able to convert chanoclavine-l to the ultimate end product fumigaclavine C (Goetz et al., 2008). As expected, our ergotryptamine-fed easE knockout controls did not show the accumulation of $A$. fumigatus downstream pathway products chanoclavine-I, festuclavine, fumigaclavine A, fumigaclavine B, or fumigaclavine $C$. The same profile was visualized in ergotryptamine-fed eas $C$ ko $A$. fumigatus cultures suggesting that ergotryptamine is not an intermediate in the ergot alkaloid pathway. If ergotryptamine was a true, on-pathway intermediate, feeding it to $A$. fumigatus eas $C$ ko should have circumvented the block in the pathway and promoted production of downstream alkaloids. Thus, ergotryptamine appears to accumulate as a pathway byproduct (or spur product) that is incapable of being enzymatically converted to chanoclavine-l or other pathway products.

Further insight to the role of ergotryptamine as a spur product may be drawn from previous studies by Kozikowski et al. (1993). These investigators found that N-Me-dienyltryptophan (Figure 1) was incorporated into later pathway products in high abundance (81-86\%), while a structurally similar allylic alcohol (Figure 4b) was incorporated in much smaller amounts (9-33\%). They also showed that NMe-dienyltryptophan was detected in trapping experiments demonstrating its role as a pathway intermediate. The allylic alcohol was not detected in trapping experiments, and together with its low incorporation rate, was rejected as an intermediate. Kozikowski et al. (1993) suggested the allylic alcohol was only incorporated into later intermediates because it was converted to N-Medienyltryptophan by a dehydration reaction. It seems likely that ergotryptamine is a derivative of the allylic alcohol rejected as an intermediated by Kozikowski et al. (1993) and cannot be enzymatically converted into later pathway products. In our A. nidulans WFC transformant the ergot alkaloid pathway abruptly ends resulting in a buildup of early pathway intermediate N-Me-DMAT which may be susceptible to spontaneous or enzyme-catalyzed oxidation and EasC-dependent decarboxylation. HPLC and ESI MS/LC analyses of $A$. nidulans WFC do not reveal the accumulation of the dienyl-version of 
ergotryptamine or other uncharacterized metabolites not seen in control strains. Moderate concentrations of ergotryptamine and dienyl-ergotryptamine would need to be synthesized and fed to ergot alkaloid producers to test this hypothesis.

Accumulation of ergotryptamine in Epichloë species and equivalency with provisional 6,7secolysergine.

HPLC analyses revealed that ergotryptamine displayed the same retention time (41 $\mathrm{min}$ ) and fluorescence properties as a molecule previously observed in E. sp. Lp1 and provisionally named 6,7secolysergine (Figures 4d and 7) (Panaccione et al., 2003). This molecule accumulated twice as abundantly in E. sp. Lp1 IpsA ko as compared to wild-type E. sp. Lp1 (Panaccione et al., 2003), so extracts of that mutant were studied to test the identity of ergotryptamine with provisional 6,7-secolysergine. Consistent with the fluorescence properties of ergotryptamine, provisional 6,7-secolysergine fluoresced intensely at $310 \mathrm{~nm}$ excitation and $410 \mathrm{~nm}$ emission settings, while it displayed a much weaker signal when analyzed with a $272 \mathrm{~nm}$ excitation and $372 \mathrm{~nm}$ emission settings. Ergot alkaloid extracts from $E$. sp. Lp1 IpsA ko-infected grasses were analyzed by ESI LC/MS to further investigate the accumulation of ergotryptamine (Figure 8). The Q Exactive high resolution ESI LC/MS used in this study revealed the protonated mass of co-eluting peaks from A. nidulans WFC and E. sp. Lp1 to be 259.1807, which varied slightly from the $259.0 \mathrm{~m} / \mathrm{z}$ value obtained with the Finnigan LCQ LC/MS used previously. Ionization of ergotryptamine leads to an abundance of ionized fragment with $\mathrm{m} / \mathrm{z}$ of 241.17 (Figure $4 \mathrm{~d}$ ). This mass corresponds to [ergotryptamine $\left.-\mathrm{H}_{2} \mathrm{O}+\mathrm{H}\right]^{+}$and also to the apparent molecular ion for 6,7-secolysergine (Panaccione et al., 2003). This fragment was also visualized during ergotryptamine analyses on the Finnigan LCQ LC/MS previously used and was found to be the dominant ion formed from selective fragmentation of ergotryptamine (data not shown). Extracts from E. sp. Lp1 IpsA ko-infected grasses displayed similar ratios of $259 \mathrm{~m} / \mathrm{z}$ parent ion to ionized $241 \mathrm{~m} / \mathrm{z}$ fragment (3.5:1) when compared to ergotryptamine from A. nidulans WFC (3.7:1) (Figure 8). These results demonstrate the accumulation of ergotryptamine as a natural product in the fungal endophyte $E$. sp. Lp1 and provide support for identity of ergotryptamine with the provisionally identified 6,7-secolysergine. We propose that the apparent molecular ion detected by Panaccione et al. (2003) was the dehydrated fragment of ergotryptamine (Figure 4d). The parent ion $\mathrm{m} / \mathrm{z} 259$ was detectable in spectra collected by Panaccione et al. (2003), but was in very low abundance, perhaps due to high deflector voltages utilized during analyses. Further, albeit anecdotal, evidence supports the creation of the dehydrated fragment $(\mathrm{m} / \mathrm{z} 241)$ as an artifact of mass spectral analyses. With the previous mass spectrometry protocol used, we detected the analogously oxidized ergot alkaloid setoclavine as its dehydrated $\mathrm{m} / \mathrm{z} 237$ ion rather than as the 
expected $\mathrm{m} / \mathrm{z} 255$ molecular ion (D.G. Panaccione, unpublished observation). The retention time of ergotryptamine in our standard HPLC gradient is consistent with it being present in the hydrated, $m / z$ 259, form. The observed retention time of ergotryptamine is close to that of similarly oxidized setoclavine and shorter (toward the more polar range) than that of N-Me-DMAT. The dehydrated, $m / z$ 241 , diene form of ergotryptamine would be expected to have a very late elution time in this gradient. Further similarities between ergotryptamine from A. nidulans WFC and the provisionally identified 6,7secolysergine from E. sp. Lp1 were revealed during purification of these compounds. Compounds from both sources required acetic acid in addition to methanol for elution from a non-end capped C18 SPE column rather than eluting in $100 \%$ methanol, which is typical of all other ergot alkaloids. Based on the accumulated data, we conclude that the molecule found in abundance in E. sp. Lp1 IpsA ko extracts is ergotryptamine.

Several other Epichloë spp. contained a metabolite that co-eluted with ergotryptamine when analyzed by HPLC (Table 2). Fungal extracts from two asexual hybrid species, Epichloë coenophiala in tall fescue (Lolium arundinaceum) and Epichloë funkii in sleepy grass (Achnatherum robustum), contained an analyte characteristic of ergotryptamine. The sexual nonhybrid Epichloë elymi produced ergotryptamine in its host Canadian wild rye (Elymus canadensis). The accumulation of alkaloid $\mathrm{X}$ appears to be species specific as we did not detect ergotryptamine in other common endophytes including Epichloë gansuensis harbored within drunken horse grass (Achnatherum inebrians) and Epichloë fescue var. lolii infected in perennial ryegrass (Lolium perenne).

\section{Agricultural perspective.}

Ergot alkaloids cause toxicosis in livestock by interacting with multiple neurotransmitter receptors. The biological activities of ergotryptamine are unknown; yet they remain of interest due to the accumulation of this metabolite in prominent grass endophytes in the genus Epichloë. Feeding preference studies utilizing various mutant Epichloë infected grasses have shown that rabbits prefer to feed on non-ergot alkaloid producing E. sp. Lp1 dmaW ko infected grasses rather than E. sp. Lp1 IpsA ko infected grasses (Panaccione et al., 2006), which contained significant quantities of chanoclavine-I and ergotryptamine. The relative contributions of ergotryptamine relative to chanoclavine-I in this feeding deterrence is unknown. Further research into the biological effects of this metabolite is needed to assess its bioactivity in subjects of importance to agriculture and medicine.

\section{References}

Coyle CM, Panaccione DG. 2005. An ergot alkaloid biosynthesis gene and clustered hypothetical genes from Aspergillus fumigatus. Appl. Environ. Microbiol. 71: 3112-3118. 
Coyle CM, Cheng JZ, O'Connor SE, Panaccione DG. 2010. An old yellow enzyme gene controls the branch point between Aspergillus fumigatus and Claviceps purpurea ergot alkaloid pathways. Appl. Environ. Microbiol. 76: 3898-3903.

Goetz KE, Coyle CM, Cheng JZ, O'Conner S, Panaccione DG. 2011. Ergot cluster-encoded catalase is required for synthesis of chanoclavine-I in Aspergillus fumigatus. Curr. Genet. 57: 201-211.

Hunter SH, Provasoli L, Schatz A, Haskins CP. 1950. Some approaches to the study of the role of metals in the metabolism of microorganisms. Am. Philos. Soc. Proc. 94: 152-170.

Kozikowski A, Chen C, Wu J, Shibuya M, Kim C, Floss H. 1993. Probing ergot alkaloid biosynthesis: Intermediates in the formation of ring C. J. Am. Chem. Soc.115: 2482-2488.

Latgé JP. 1999. Aspergillus fumigatus and aspergillosis. Clin. Microbial. Rev. 12: 310-350.

Leuchtmann A, Bacon CW, Schardl CL, White JF, Tadych M. 2014. Nomenclatural realignment of Neotyphodium species with genus Epichloë. Mycologia 106: 202-215.

Lorenz N, Olšovská J, Šulc M, Tudzynski P. 2010. Alkaloid cluster gene ccsA of the ergot fungus Claviceps purpurea encodes chanoclavine I synthase, a flavin adenine dinucleotide-containing oxidoreductase mediating the transformation of $\mathrm{N}$-methyl-dimethylallyltryptophan to chanoclavine. Appl. Environ. Microbiol.76: 1822-1830.

Panaccione DG, Johnson RD, Wang J, Young CA, Damrongkool P, Scott B, Schardl CL. 2001. Elimination of ergovaline from a grass-Neotyphodium endophyte symbiosis by genetic modification of the endophyte. Proc. Natl. Acad. Sci. USA 98: 12820-12825.

Panaccione DG, Tapper BA, Lane GA, Davies E, Fraser K. 2003. Biochemical outcome of blocking the ergot alkaloid pathway of a grass endophyte. J. Agric. Food. Chem. 51: 6429-6437.

Panaccione DG, Cipoletti JR, Sedlock AB, Blemings KP, Schard CL, Machado C, Seidel GE. 2006. Effects of ergot alkaloids of food preference and saiety in rabbits, as assessed with gene knockout endophytes in perennial ryegrass (Lolium perenne). J. Agric. Food. Chem. 54: 4582-4587.

Panaccione DG. 2010. Ergot alkaloids. The Mycota, Vol. X, Industrial Applications, 2nd Edition (Ed. M. Hofrichter), Berlin-Heidelburg: Springer-Verlag, 195-214.

Panaccione DG, Ryan KL, Schardl CL, Florea S. 2012. Analysis and modification of ergot alkaloid profiles in fungi. Methods Enzymol. 515: 267-290.

Rigbers O, Li SM. 2008. Ergot alkaloid biosynthesis in Aspergillus fumigatus overproduction and biochemical characterization of a 4-dimethylallyltryptophan N-methyltransferase. J. Biol. Chem. 283: 26859-26868.

Ryan KL, Moore C, Panaccione DG. 2013. Partial reconstruction of the ergot alkaloid pathway by heterologous gene expression in Aspergillus nidulans. Toxins 5: 445-455.

Schardl CL, Young CA, Hesse U, Amyotte SG, Andreeva K, Calie PJ, Fleetwood DJ, Haws DC, Moore N, Oeser B, Panaccione DG, Schweri KK, Voisey CR, Farman ML, Jaromczyk JW, Roe BA, O'Sullivan DM, Scott B, Tudzynski P, An Z, Arnaoudova EG, Bullock CT, Charlton ND, Chen L, Cox M, Dinkins RD, Florea S, Glenn AE, Gordon A, Güldener U, Harris DR, Hollin W, Jaromczyk J, Johnson RD, Khan AK, Leistner E, Leuchtmann A, Li C, Liu JG, Liu J, Liu M, Mace W, Machado C, Nagabhyru P, Pan J, Schmid J, Sugawara K, Steiner U, Takach JE, Tanaka E, Webb JS, Wilson EV, Wiseman JL, Yoshida RY, Zeng Z. 2013a. Plant-symbiotic fungi as chemical engineers: multi-genome analysis of the Clavicipitaceae reveals dynamics of alkaloid loci. Plos Genetics 9: e1003323. 
Schardl CL, Florea S, Pan J, Nagabhyru P, Bec S, Calie PJ. 2013b. The epichloae: alkaloid diversity and roles in symbiosis with grasses. Curr. Opin. Plant Biol. 16: 480-488.

Scigelova M, Macek T, Minghetti A, Mackova M, Sedmera P, Prikrylova V, Kren V. 1995.

Biotransformation of ergot alkaloids by plant cell cultures with high peroxidase activity. Biotechnol. Lett. 17: 1213-1218.

Scudamore KA, Livesey CT. 1997. Occurrence and significance of mycotoxins in forage crops and silage: a review. J. Sci. Food Agric. 77: 1-17.

Strickland JR, Brown KR, Aiken GE, Klotz JL, Flythe MD. 2012. Ergot alkaloids: toxicokinetics and vascular effects. International Symposium on Fungal Endophytes of Grasses; (Ed. Young CA, Aiken GE, McCulley RL, Strickland JR, Schardl CL), Noble Foundation: Ardmore, OK, 14-19.

Thompson RW, Fribourg HA, Waller JC, Sanders WI, Reynolds JH, Phillips JM, Schmidt SP, Crawford RJ, Allen VG, Faulkner DB. 1993. Combined analysis of tall fescue steer grazing studies in the eastern United States. J. Anim. Sci. 71: 1940-1946.

Unsöld Al, Li SM. 2005. Overproduction, purification and characterization of FgaPT2, a dimethylallyltryptophan synthase from Aspergillus fumigatus. Microbiol. 115: 1499-1505.

Wang J, Machado C, Panaccione DG, Tsai H-F, Schardl CL. 2004. The determinate step in ergot alkaloid biosynthesis by an endophyte of perennial ryegrass. Fungal Genet. Biol. 41: 189-198. 
Table 1. ${ }^{1} \mathrm{H}$ and ${ }^{13} \mathrm{C}$ NMR data (chemical shifts and coupling constants) of alkaloid X in DMSO- $d_{6}$

\begin{tabular}{ccccccc}
\hline Proton & $\delta_{\mathrm{H}} / \mathrm{ppm}$ & Carbon & $\delta_{\mathrm{X}} / \mathrm{ppm}$ & \multicolumn{2}{c}{$\mathrm{J}_{\mathrm{HH}}(\mathrm{n}=3,4)$} & $\mathrm{J} / \mathrm{Hz}$ \\
\hline $\mathrm{H} 1$ & 10.83 & $\mathrm{C} 2$ & 123.75 & $3 \mathrm{~J}$ & $\mathrm{H} 1-\mathrm{H} 2$ & 2.4 \\
$\mathrm{H} 2$ & 7.1 & $\mathrm{C} 3$ & 113.8 & $3 \mathrm{~J}$ & $\mathrm{H} 6-\mathrm{H} 7$ & 7.5 \\
$\mathrm{H} 6$ & 7.2 & $\mathrm{C} 4$ & 124.23 & $3 \mathrm{~J}$ & $\mathrm{H} 7-\mathrm{H} 8$ & 7.9 \\
$\mathrm{H} 7$ & 6.99 & $\mathrm{C} 5$ & 133.25 & $4 \mathrm{~J}$ & $\mathrm{H} 6-\mathrm{H} 8$ & 0.8 \\
$\mathrm{H} 8$ & 7.01 & $\mathrm{C} 6$ & 110.69 & $3 \mathrm{~J}$ & $\mathrm{H} 10-\mathrm{H} 11$ & 7.5 \\
$10-\mathrm{CH}_{2}$ & 2.98 & $\mathrm{C} 7$ & 122.93 & $3 J_{\text {trans }}$ & $\mathrm{H} 14-\mathrm{H} 15$ & 15.8 \\
$11-\mathrm{CH}_{2}$ & 2.77 & $\mathrm{C} 8$ & 115.87 & & & \\
$\mathrm{~N}-\mathrm{CH}_{3}$ & 2.33 & $\mathrm{C} 9$ & 136.74 & & & \\
$\mathrm{H} 14$ & 6.2 & $\mathrm{C} 10$ & 27.1 & & & \\
$\mathrm{H} 15$ & 7.17 & $\mathrm{C} 11$ & 53.84 & & & \\
$\mathrm{H} 17 / \mathrm{H} 18$ & 1.31 & $\mathrm{C} 13$ & 35.74 & & & \\
$\mathrm{OH}$ & $\mathrm{n} .0$. & $\mathrm{C} 14$ & 129.05 & & & \\
$\mathrm{NH}$ & n.0. & $\mathrm{C} 15$ & 140.25 & & & \\
& & $\mathrm{C} 16$ & 69.62 & & \\
& & $\mathrm{C} 17 / \mathrm{C} 18$ & 30.38 & & \\
\hline
\end{tabular}

n.o. = not observed 
Table 2. Ergotryptamine concentrations in various Epichloë sp.-infected plants

\begin{tabular}{llcc}
\hline Plant & Fungus & $\begin{array}{c}\text { Number of } \\
\text { samples }\end{array}$ & $\begin{array}{c}\text { Ergotryptamine } \\
(\mu \mathrm{g} / \mathrm{g} ; \text { mean }+ \text { - SE) }\end{array}$ \\
\hline Lolium arundinaceum & E. coenophiala isolate 4163 & 3 & $0.16 \pm .04$ \\
Lolium arundinaceum & E. coenophiala isolate 19 & 5 & $0.27 \pm .08$ \\
Achnatherum robustum & E. funkii & 23 & $0.32 \pm .03$ \\
Elymus canadensis & E. elymi & 2 & $1.76 \pm .22$ \\
Lolium perenne & E. sp. Lp1 & 3 & $0.90 \pm .20$ \\
Achnatherum inebrians & E. gansuensis & 9 & n.d. ${ }^{b}$ \\
Lolium perenne & E. festucae var. lolii & 8 & n.d. \\
\hline
\end{tabular}

${ }^{a}$ All assays were conducted in the same way and using the sample instrumentation; though plants were of different ages and harvested separately.

${ }^{b}$ n.d. $=$ not detected 
<smiles>CCCc1cccc2[nH]cc(CC(N)C(=O)O)c12</smiles>

DMAPP Tryptophan DMAT

N-Me-DMAT

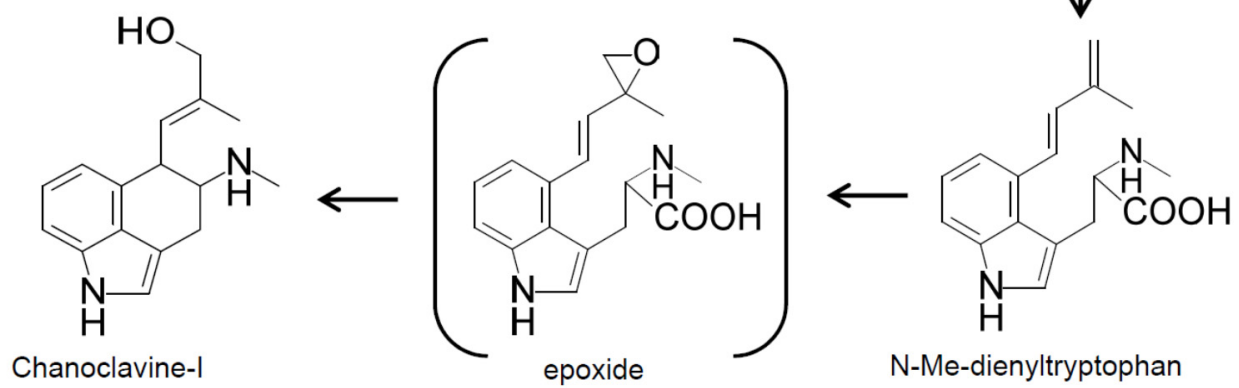

Figure 1: Early steps of ergot alkaloid synthesis, redrawn from Kozikowski et al. (1993). Compound in parentheses is hypothetical. DMAPP= dimethlyallypyrophosphate, DMAT= dimethylallyltryptophan, $\mathrm{N}$ Me-DMAT $=\mathrm{N}$-methyl-dimethylallyltryptophan 


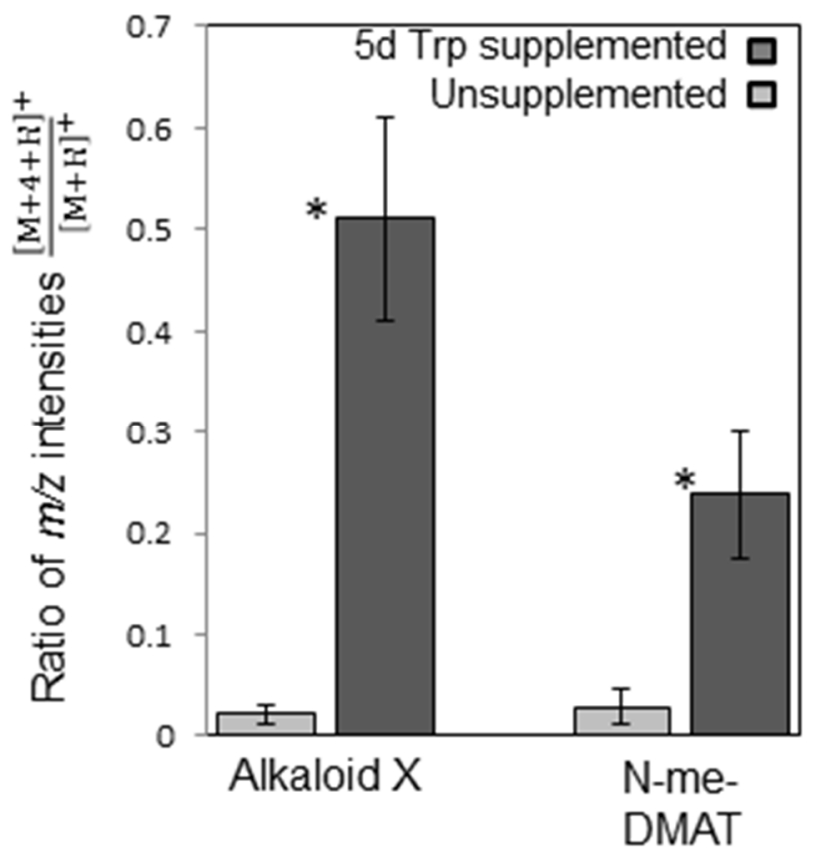

Figure 2: Alkaloid X as a tryptophan derivative. A. nidulans WFC cultures were incubated on ST medium or ST medium supplemented with L-tryptophan-5d (indole $5 \mathrm{~d}$ ) followed by an LC/MS analysis.

Incorporation of four tryptophan-derived deuteriums into alkaloid $\mathrm{X}$ and known intermediate $\mathrm{N}-\mathrm{Me}$ DMAT was measured by recording intensity values for compounds with a $\mathrm{m} / \mathrm{z}$ value of 259.0 and 263.0 (for alkaloid X) and $m / z$ values of 287.0 and 291.0 (for N-Me-DMAT). Error bars represent the mean standard error of intensities. Student's t-test was performed, and statistical significance is represented by $*(P<0.05)$. 


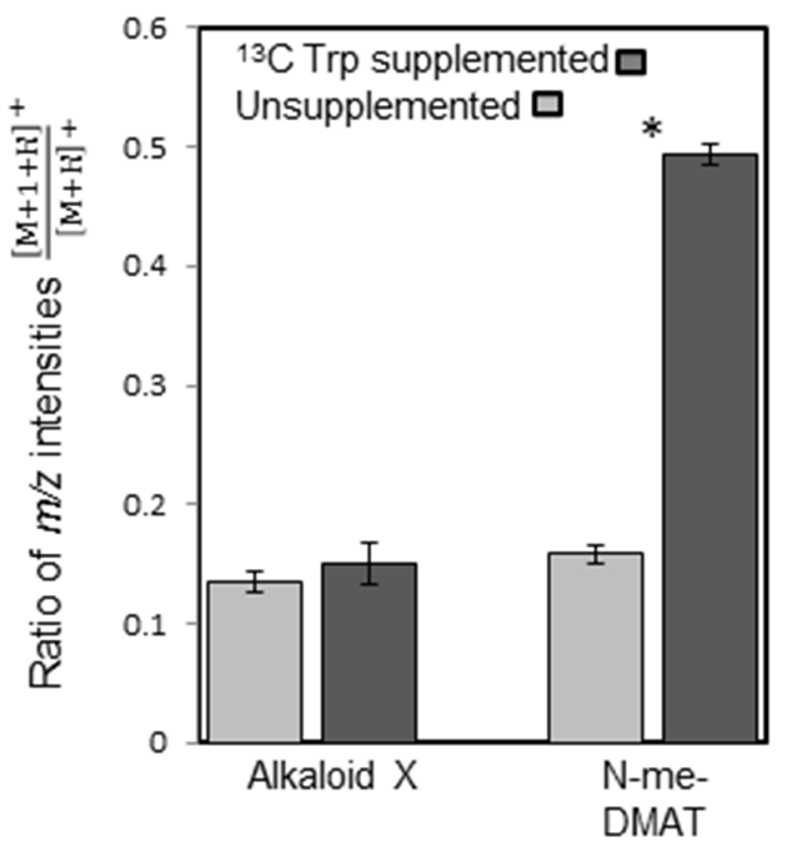

Figure 3: Alkaloid X lacks tryptophan-derived carboxyl group. A. nidulans WFC cultures were incubated on ST medium or ST medium supplemented with L-tryptophan- $-1{ }^{13} \mathrm{C}$. The presence of the carboxyl group in alkaloid $\mathrm{X}$ and intermediate $\mathrm{N}$-Me-DMAT was elucidated by recording intensity values for compounds with a $\mathrm{m} / \mathrm{z}$ value of 259.0 and 260.0 (for alkaloid $X$ ) and compounds with $\mathrm{m} / \mathrm{z}$ values of 287.0 and 288.0 (for N-Me-DMAT). Error bars represent the mean standard error of intensities. Student's t-test was performed. Statistical significance is represented by * $(P<0.05)$. 

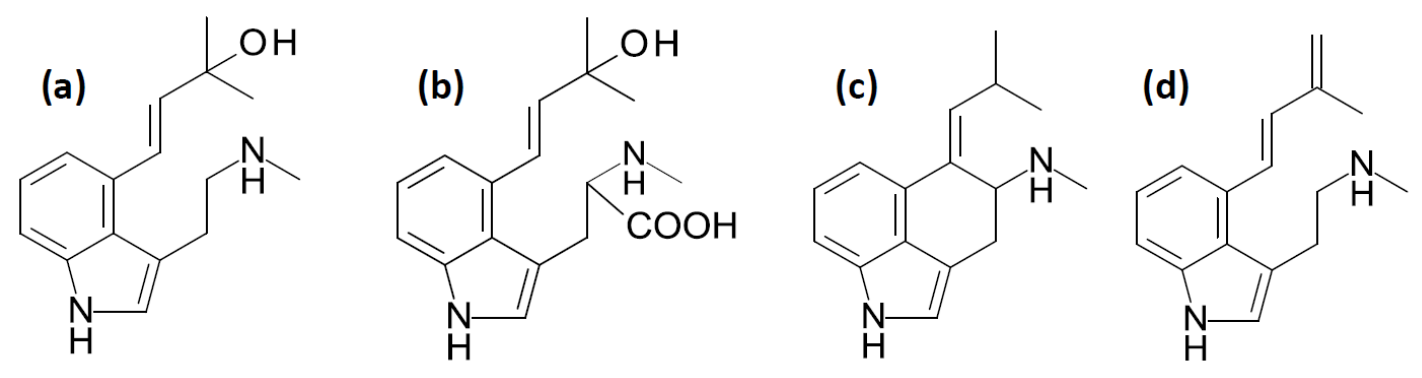

Figure 4: Chemical structures. (a) alkaloid X (ergotryptamine). (b) allylic alcohol of N-Me-

dienyltryptophan (Kozikowski et al., 1993). (c) provisional 6,7 secolysergine (Panaccione et al., 2003). (d) $\mathrm{m} / \mathrm{z} 241$ fragment from ergotryptamine ionization. 


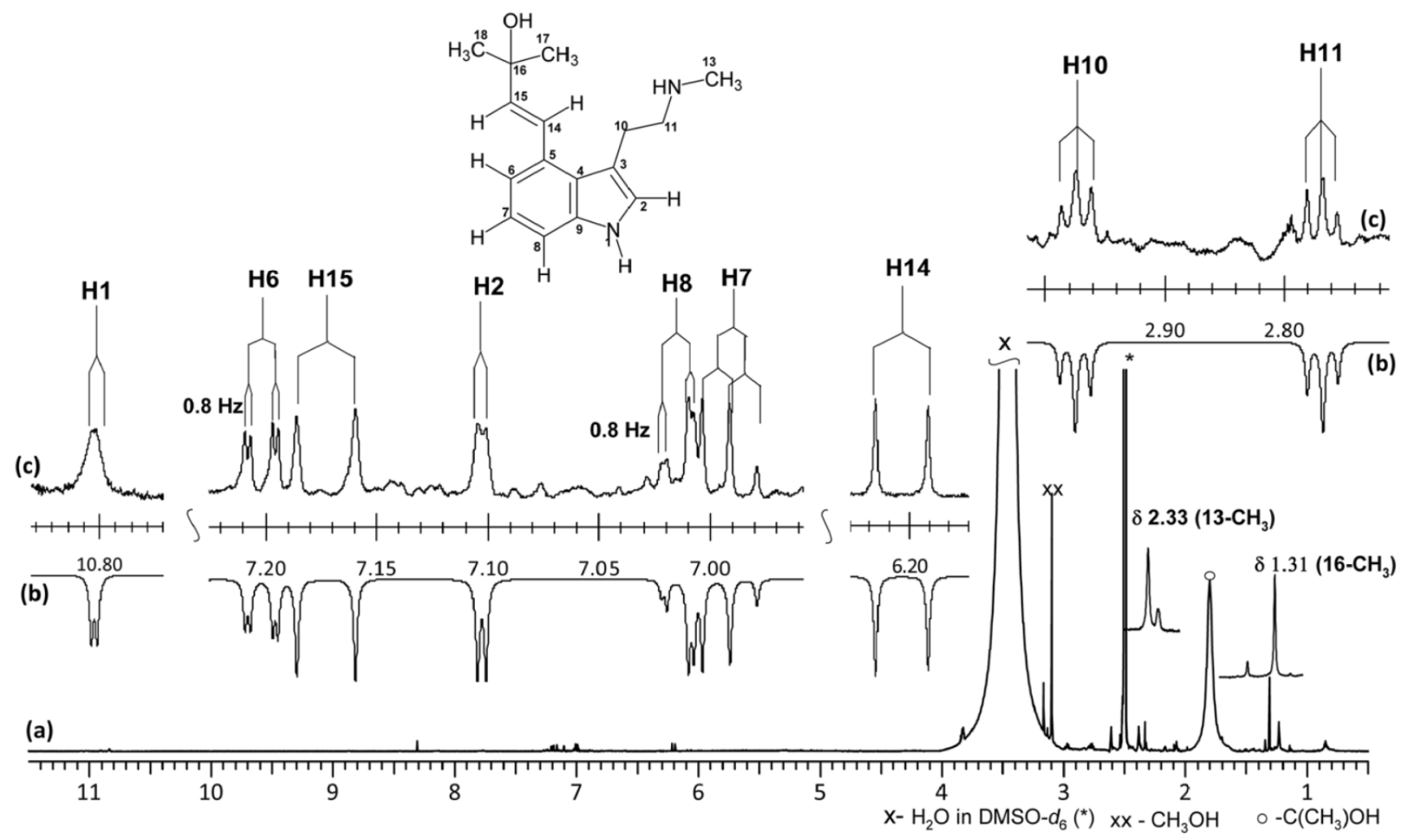

Figure 5: ${ }^{1} \mathrm{H}$ NMR spectrum (a) of alkaloid $\mathrm{X}$ in DMSO- $d_{6}$. The inserted spectra $(b-c)$ are the back to back representation of the expanded portions of the experimental (c) and calculated (b) ${ }^{1} \mathrm{H}$ NMR spectrum. 


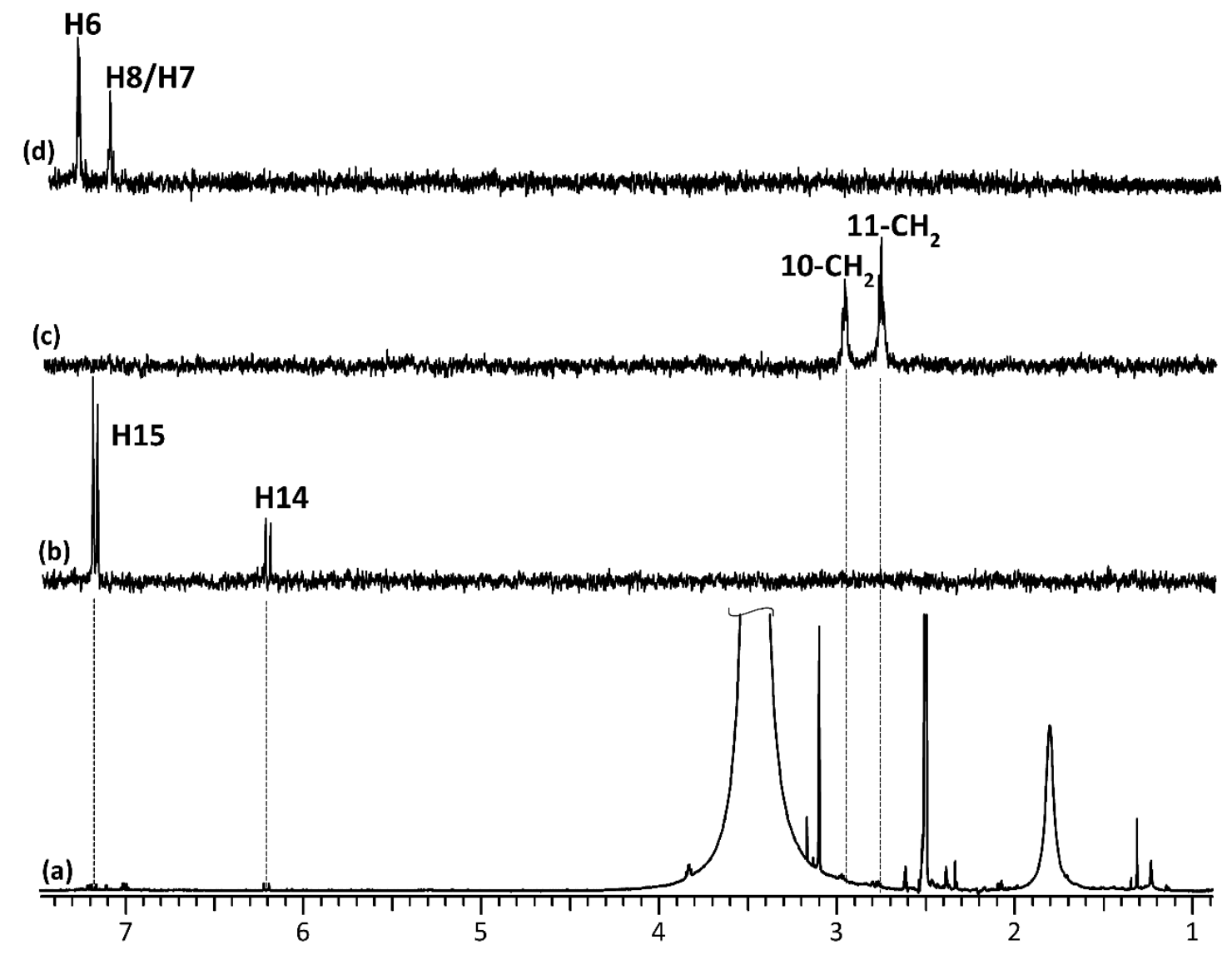

Figure 6: Experimental 1D TOCSY subspectra $(b-d$, mixing time $=80 \mathrm{~ms})$ of alkaloid X. Control ${ }^{1} \mathrm{H} \mathrm{NMR}$ spectrum (a); Subspectrum-b, selective excitation of $\mathrm{H} 14$ at d 7.17 Subspectrum -c, selective excitation of 11- $\mathrm{CH}_{2}$ at $\mathrm{d}$ 2.77; Subspectrum-d, selective excitation of $\mathrm{H6}$ at 7.20. 


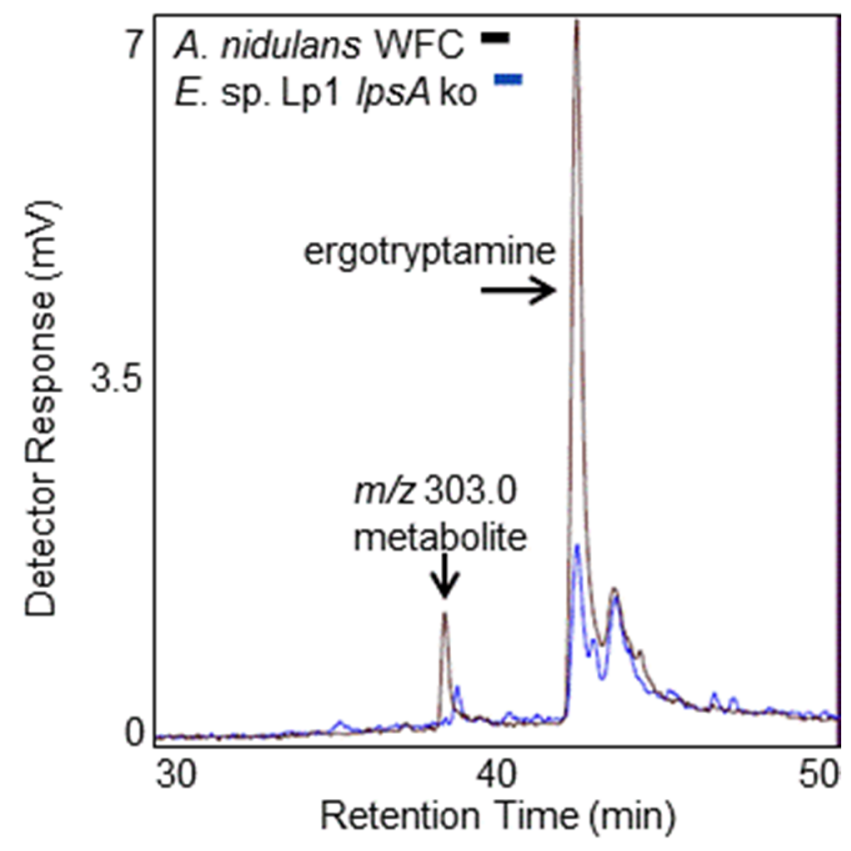

Figure 7: Accumulation of alkaloid X (ergotryptamine) in Epichloë spp. Ergot alkaloids produced by $A$. nidulans WFC (black) and IpsA ko E. sp. Lp1 infected perennial ryegrass (blue) were analyzed by HPLC with fluorescence detector setting $310 \mathrm{~nm}$ excitation and $410 \mathrm{~nm}$ emissions. 


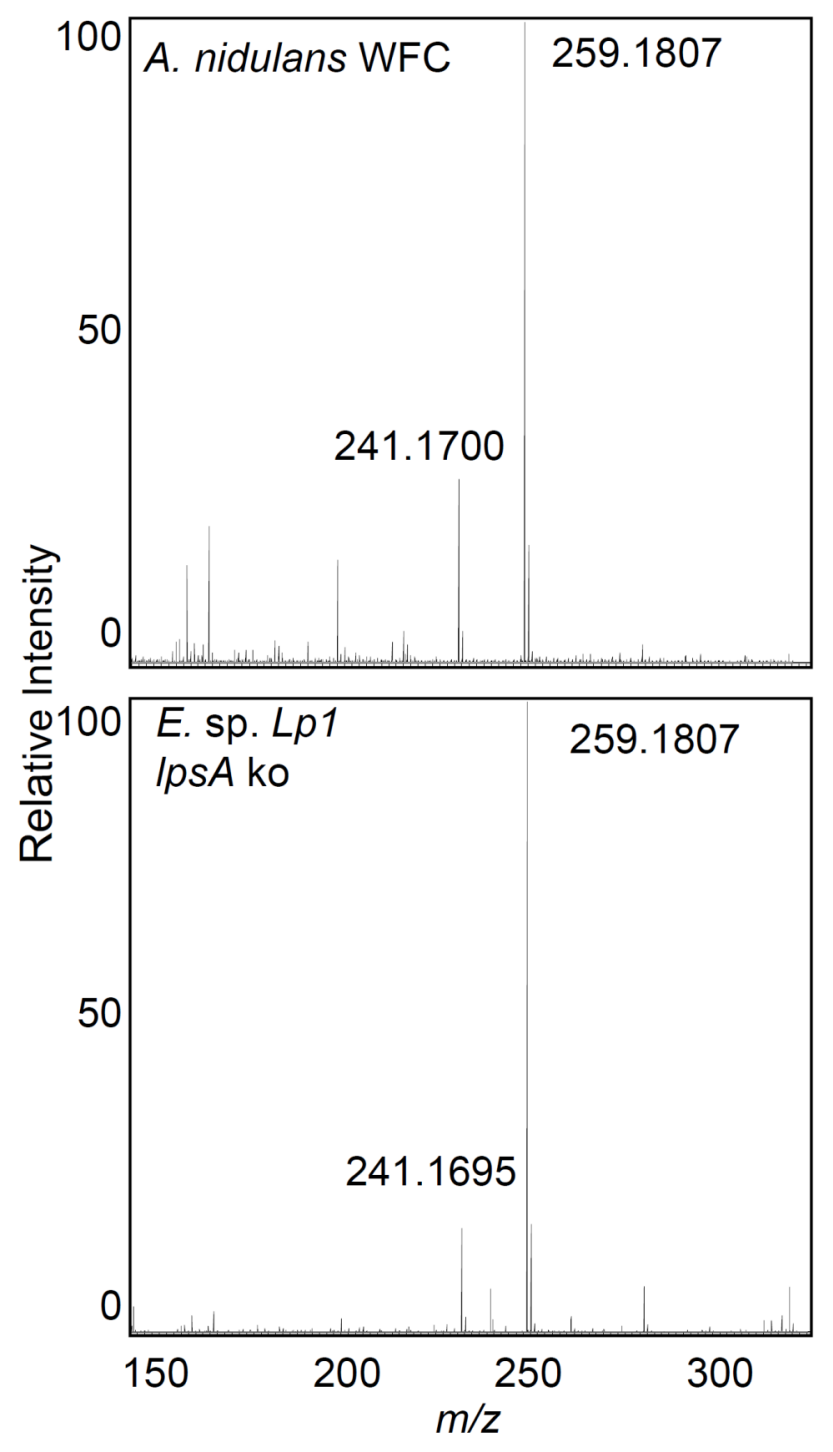

Figure 8: LC/MS spectra of ergotryptamine in A. nidulans WFC and IpsA ko E. sp. Lp1 infected perennial ryegrass. $m / z 259.18$ = ergotryptamine parent ion; $m / z 241.17$ = dehydrated ergotryptamine ion fragment. 


\section{Chapter 4}

Heterologous expression of ergot alkaloid synthesizing genes in Aspergillus nidulans: additional mutants 


\section{Introduction}

Continuing the work initiated in chapter 2 and 3 of my thesis, various gene combinations of dmaW, easC, and easE were transformed into Aspergillus nidulans in order to better understand the roles of EasC and EasE. DmaW performs the first step of ergot alkaloid synthesis yielding DMAT, while EasF executes the second reaction converting DMAT to N-Me-DMAT (Wang et al., 2004; Unsöld and Li, 2005; Rigbers and Li, 2008). EasE and EasC are required for chanoclavine-I synthesis; however their specific roles are unknown (Goetz et al., 2011; Lorenz et al., 2010). The formation of ergotryptamine from transformant $A$. nidulans WFC (described in Ch. 3 ) suggests EasC is necessary for alkaloid decarboxylation; while bioinformatics indicate that EasE is responsible for closure of the third ergoline ring. Continuing this study, I have generated several $A$. nidulans mutants expressing $d m a W$, easC and/or easE to determine if EasE and EasC are capable of acting nonspecifically on DMAT to yield novel ergot alkaloids. Four additional transformants were created: $A$. nidulans W (containing dmaW; serving as a control strain), A. nidulans WC (expressing dmaW and eas $C$ ), $A$. nidulans WE (expressing dmaW and easE), and $A$. nidulans WEC (expressing dmaW, easE and easC).

\section{Methods}

PCR amplification: DNA was extracted from A. fumigatus isolate Af293 using the protocol described by Richards et al. (1994). A $7.1 \mathrm{~kb}$ fragment containing dmaW, easE, easC, was amplified by PCR, primed with oligonucleotides Primer1 ( ${ }^{5^{\prime}}$ TACCTATACCTAATCGAAGCCGCACGCAGTGCACC ${ }^{3}$ ) and Primer4 $\left(5^{\prime}\right.$ ATATCTGACCGACCATCCCTTACAGCCATGTGCAGC ${ }^{3}$ ). A fragment containing dmaW and easE, was amplified

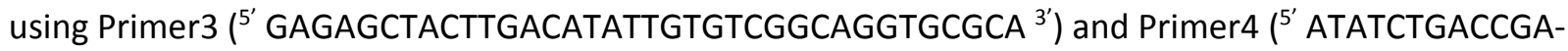
CCATCCCTTACAGCCATGTGCAGC ${ }^{3}$ ) yielding a $4.5 \mathrm{~kb}$ fragment. A $4.8 \mathrm{~kb}$ fragment containing dmaW and eas $C$ was amplified using Primer1 ( ${ }^{5^{\prime}}$ TACCTATACCTAATCGAAGCCGCACGCAGTGCACC $\left.{ }^{3}\right)$ and Primer5 $\left({ }^{5^{\prime}}\right.$ CTACTCCATTGTCCTGTGAGTTG ${ }^{3}$ ). A $2.2 \mathrm{~kb}$ fragment containing dmaW was amplified utilizing Primer3

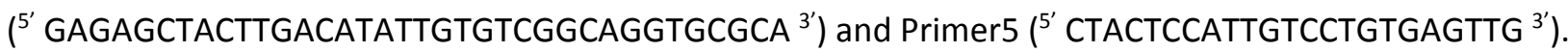
Twenty five $\mu \mathrm{L}$ reactions contained $1 \mathrm{X}$ LongAmp Taq reaction buffer, $200 \mu \mathrm{M}$ of each deoxynucleotide triphosphate, $1 \mu \mathrm{M}$ of each primer, and 2.5 units LongAmp Taq DNA polymerase (New England BioLabs). Each reaction began with an initial denaturing step of 3 minutes at $94{ }^{\circ} \mathrm{C}$, followed by 35 cycles of 30 seconds at $94{ }^{\circ} \mathrm{C}$ and 10 minutes at $65^{\circ} \mathrm{C}$, with a final extension of 5 minutes at $65^{\circ} \mathrm{C}$.

Fungal transformation and screening: Preparation of $A$. nidulans isolate FGSC A767 protoplasts and fungal transformations were conducted according to Ryan et al. (2013). Each transformant was screened by PCR for the presence of $d m a W$, easF, eas $C$, and easE. The $d m a W$ gene was primed with internal gene 
binding oligos dmaWF ( $\left(5^{\prime}\right.$ TTGATCTGGAGTGGTTCCGC $\left.{ }^{3}\right)$ and dmaWR ( ${ }^{5}$ CGTTCATGCCGAAGGTTGTG $\left.{ }^{3}\right)$ yielding a $651 \mathrm{bp}$ fragment. Amplification of easE was primed with easEF ( ${ }^{5}$ CCAGATACATTGCCATCGCATG $^{3^{\prime}}$ ) and easER $\left(5^{\prime}\right.$ TGTTCCAACTGCTTGGCCAGAT $\left.{ }^{3}\right)$, which amplified an 897 bp fragment. Primers for amplifying eas $C$ were easCF $\left({ }^{5}\right.$ GAATTCGAGGTATTGATCTCC ${ }^{3}$ ) and easCR $\left({ }^{5}\right.$ AGCCAGGCAAAGATCCATA$\mathrm{GTT}^{3}$ ) which amplify a $1036 \mathrm{bp}$ fragment. The eas F gene was primed with easFF ( ${ }^{5}$ AAGTTGTCGAAGGTCTCACGAA $\left.{ }^{3}\right)$ and easFR $\left({ }^{\prime}\right.$ GTGATTAGAGATGCTTCTGTC $\left.{ }^{3}\right)$, producing an 820 bp fragment. Twenty five $\mu \mathrm{L}$ reactions contained $1 \times$ GoTaq Flexi buffer, $200 \mu \mathrm{M}$ deoxynucleotide triphosphate, $1.5 \mathrm{mM} \mathrm{MgCl} 2,1 \mu \mathrm{M}$ of each primer, and 2.5 units of Taq DNA polymerase (Promega, Madison, WI, USA). PCR conditions began with an initial denaturing step of $3 \mathrm{~min}$ at $94{ }^{\circ} \mathrm{C}$, followed by 35 cycles of $30 \mathrm{~s}$ at $94{ }^{\circ} \mathrm{C}$ followed by $30 \mathrm{~s}$ at $55^{\circ} \mathrm{C}$, and $1 \mathrm{~min}$ and $30 \mathrm{~s}$ at $72^{\circ} \mathrm{C}$, followed by a final extension of $5 \mathrm{~min}$ at $72{ }^{\circ} \mathrm{C}$.

HPLC analysis: Positive transformants were analyzed by HPLC for ergot alkaloid production. Ergot alkaloids were extracted by incubating one $\mathrm{cm}^{2}$ of spore rich fungal matter in $400 \mu \mathrm{L}$ of methanol for 30 minutes, followed by centrifugation for extract clarification. Extracts were then transferred into a C18 column (Prodigy 5- $\mu \mathrm{m}$ ODS3 (150 mm by $4.6 \mathrm{~mm}$ ); Phenomenex, Torrance, CA, USA) and exposed to a binary gradient of solution $\mathrm{A}(5 \%(\mathrm{v} / \mathrm{v})$ acetonitrile plus $95 \%(\mathrm{v} / \mathrm{v})$ aqueous $50 \mathrm{mM}$ ammonium acetate) and solution B ( $75 \%$ acetonitrile plus $25 \%$ aqueous $50 \mathrm{mM}$ aqueous ammonium acetate) as described by Ryan et al. (2013).

LC/MS analysis: Ergot alkaloids were extracted following the procedure listed above; however, clarified extracts were concentrated in vacuum centrifuge to $50 \mu \mathrm{L}$ for LC/MS analysis. Ten $\mu \mathrm{L}$ of concentrated sample were injected into a Finnigan LCQ DecaXP plus mass spectrometer. Metabolites were separated on a C18 column (Phenomenex 4- $\mu \mathrm{m}$ polar-RP $(150 \mathrm{~mm}$ by $2 \mathrm{~mm}$ )) by combining mobile phases A ( $5 \%$ acetonitrile, $0.1 \%$ formic acid) and B (75\% acetonitrile, $0.1 \%$ formic acid) following the protocol described by Ryan et al. (2013).

\section{Results and Discussion}

A. nidulans transformants W, WC, WE, and WEC were analyzed by HPLC for ergot alkaloid production. As expected, due to the presence of $d m a W$, all transformants produced an analyte that coeluted with DMAT at 43 minutes visualized at fluorescence setting $272 \mathrm{~nm}$ excitation and $372 \mathrm{~nm}$ emissions (Figure 1). DmaW is the first enzyme in the pathway producing DMAT from substrates tryptophan and DMAPP. The accumulation of DMAT in each strain was predicted due to the expression of dmaW. ESI LC/MS analyses determined the analyte had an $m / z$ of 273.0 consistent with [DMAT $+\mathrm{H}^{+}$ (Figure 2). 
All transformants also accumulated an uncharacterized ergot alkaloid not seen in wild-type $A$. nidulans which eluted from the HPLC column at 37 minutes (Figure 1). The ergot alkaloid was visualized at a fluorescence setting of $310 \mathrm{~nm}$ excitation and $410 \mathrm{~nm}$ emissions which is characteristic of alkaloids with a 9, 10 carbon double bond (Panaccione et al., 2012). ESI LC/MS analyses revealed the compound to have an $\mathrm{m} / \mathrm{z}$ of 289.0 , consistent with [DMAT $+\mathrm{OH}+\mathrm{H}^{+}$(Figure 2). In $\mathrm{Ch} .3$ of my thesis it was revealed that N-Me-DMAT can be spontaneously hydroxylated when incubated in liquid ST medium producing the provisional compound in Figure 3. Based on genetic data, fluorescence properties, and mass spectral data, we propose that the compound is oxidized DMAT (Figure 3). The retention time of this compound (37 minutes) is consistent with its polarity properties.

Ergot alkaloid profiles were consistent among all transformants. Novel ergot alkaloids, besides hydroxylated DMAT, were not detected in any mutant strains suggesting that EasC and EasE cannot use DMAT as a substrate. Though novel ergot alkaloids were not observed in individual strains, the accumulation of hydroxylated DMAT added to our understanding alkaloid synthesis and interpretation of ergot profiles.

\section{References}

Goetz KE, Coyle CM, Cheng JZ, O'Conner S, Panaccione DG. 2011. Ergot cluster-encoded catalase is required for synthesis of chanoclavine-I in Aspergillus fumigatus. Curr. Genet. 57: 201-211.

Lorenz N, Olšovská J, Šulc M, Tudzynski P. 2010. Alkaloid cluster gene ccsA of the ergot fungus Claviceps purpurea encodes chanoclavine I synthase, a flavin adenine dinucleotide-containing oxidoreductase mediating the transformation of $\mathrm{N}$-methyl-dimethylallyltryptophan to chanoclavine. Appl. Environ. Microbiol.76: 1822-1830.

Panaccione DG, Ryan KL, Schardl CL, Florea S. 2012. Analysis and modification of ergot alkaloid profiles in fungi. Methods Enzymol. 515: 267-290.

Ryan KL, Moore C, Panaccione DG. 2013. Partial reconstruction of the ergot alkaloid pathway by heterologous gene expression in Aspergillus nidulans. Toxins 5: 445-455.

Richards, E, Reichardt M, Rogers S. 2001. Preparation of genomic DNA from plant tissue. Curr. Protoc. Mol. Biol. 27: 2.3.1-2.3.7.

Rigbers O, Li SM. 2008. Ergot alkaloid biosynthesis in Aspergillus fumigatus overproduction and biochemical characterization of a 4-dimethylallytryptophan N-methyltransferase. J. Biol. Chem. 283: 26859-26868.

Unsöld IA, Li S-M. 2005. Overproduction, purification and characterization of FgaPT2, a dimethylallyltryptophan synthase from Aspergillus fumigatus. Microbio. 151: 1499-1505.

Wang J, Machado C, Panaccione DG, Tsai H-F, SchardI CL. 2004. The determinate step in ergot alkaloid biosynthesis by an endophyte of perennial ryegrass. Fungal Genet. Biol. 41: 189-198. 

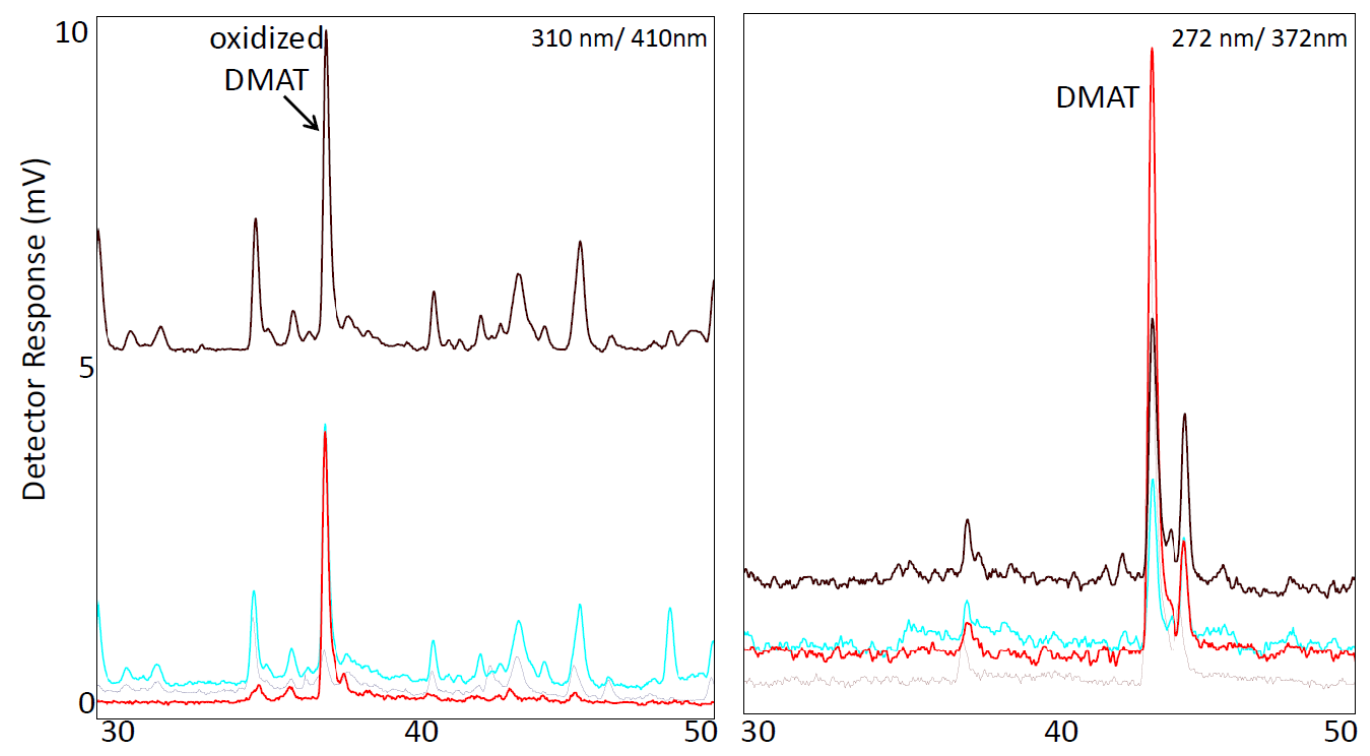

Figure 1: Ergot alkaloid profiles of $A$. nidulans transformants. A. nidulans W (black), A. nidulans WE (red), A. nidulans WC (blue), A. nidulans WEC (gray). 

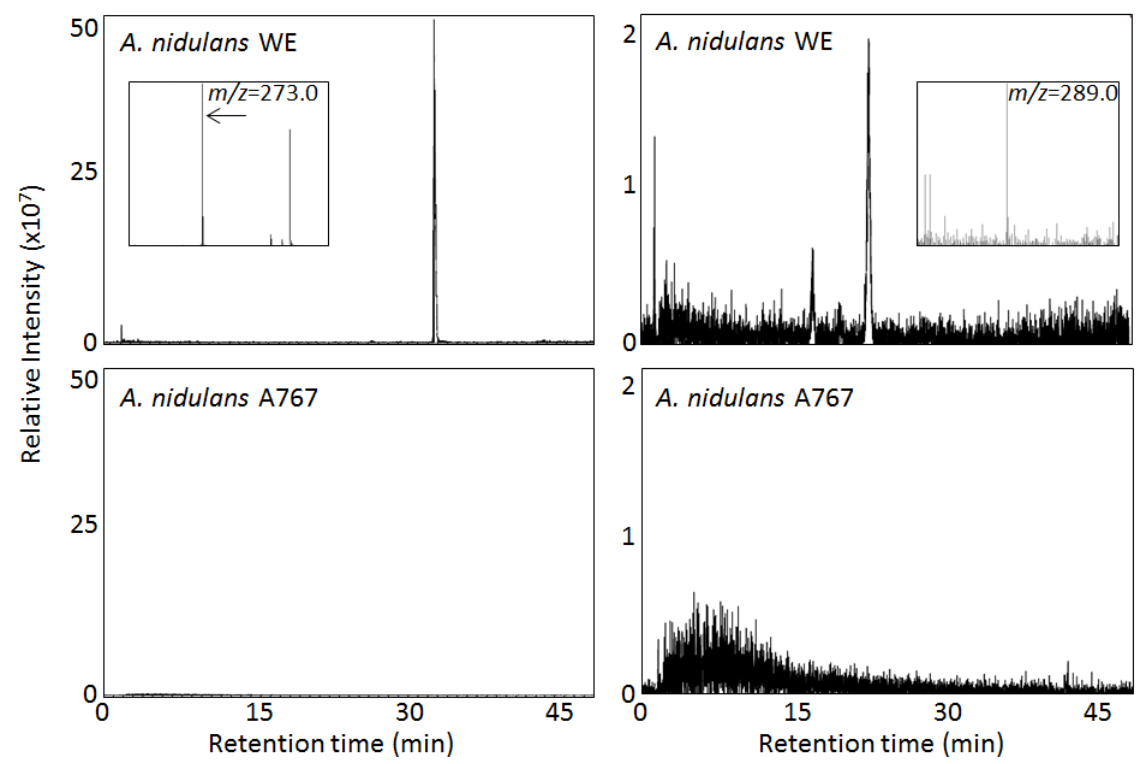

Figure 2: LC-MS analysis of mutant $A$. nidulans extracts. Chromatograms display presence and elution time of molecules with an $m / z$ value of $273 \pm 0.5$ (left column) or $289 \pm 0.5$ (right column).

Chromatograms were normalized to the intensity of the highest peak in the $A$. nidulans WE trace for each analyte. 


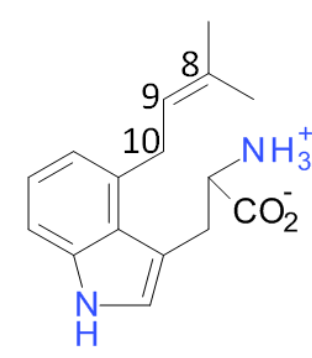

DMAT

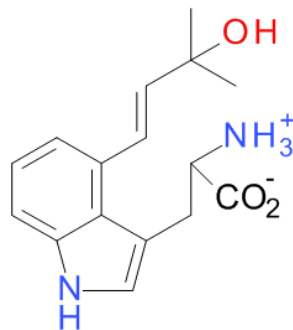

Oxidized

DMAT

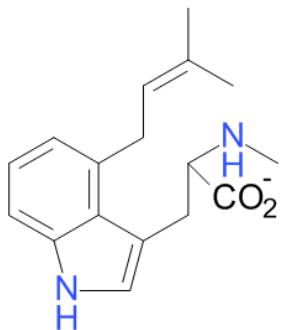

N-Me-DMAT

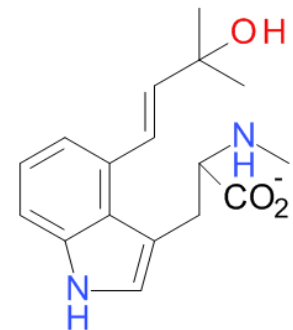

Oxidized

N-Me-DMAT

Figure 3 Chemical Structures. 


\section{Chapter 5}

Investigating the involvement of easA in lysergol and cycloclavine synthesis 


\section{Introduction}

Morning glories are capable of forming a mutualistic symbiosis with ergot alkaloid-producing fungi (Steiner et al. 2006). Two particular morning glory endophytes have been sequenced and aligned in the Clavicipitaceae, Periglandula ipomoeae which infects Ipomoea asarifolia and Periglandula turbinae which infects Turbina corymbosa (Steiner et al., 2011). Several morning glory species associate with fungi that produce unique ergot alkaloids. Ipomoea parasitica associates with a Periglandula-like fungus capable of synthesizing lysergol (Figure 1), a popular compound currently used as a bioenhancer in the development of antibiotics (Khanuja 2006; Maurya et al., 2013). Ipomoea hildebrandtii harbors a Periglandula-like fungus that synthesizes the unique alkaloid, cycloclavine (Figure 1) (Beaulieu et al., 2013; Panaccione et al., 2014). Though no biological activities are known for cycloclavine, this compound has sparked interest due to its high energy cyclopropane bond.

The pathways for the formation of lysergol and cycloclavine are unknown; however, both alkaloids are produced downstream of chanoclavine. Lysergine, the precursor of lysergol, is a double bond isomer of agroclavine containing a 9,10 carbon double bond, as opposed to the 8,9 double bond in agroclavine (Figure 1). Cycloclavine differs from lysergol and agroclavine in having an unusual 8,10 cyclopropane bond.

EasA has previously been described as a structural branch point in the ergot pathway responsible for (along with EasG) either agroclavine or festuclavine synthesis (Coyle et al., 2010; Cheng et al., 2010). The easA gene encodes an oxidoreductase responsible for reducing the alkene in chanoclavine-I aldehyde thereby enabling closure of fourth ergoline ring. Some variants of EasA are capable of fully reducing the 8,9 carbon double bond to yield festuclavine, while other fungi contain a variant easA allele incapable of double bond reduction yielding agroclavine.

I. hildebrandtii extracts normally contain festuclavine while I. parasitica extracts accumulate ergopeptines (agroclavine derivatives) indicating both endophytes contain at least one functional copy of the easA gene (Beaulieu et al., 2013; Amor-Prats and Harborne, 1993). Since EasA is a pathway branch point and both cycloclavine and lysergine are structurally similar to agroclavine with the exception of the 8,9 double bond, I hypothesized that a second, variant copy of the easA gene in both endophytes is responsible for the formation of lysergine and cycloclavine.

\section{Methods}


DNA extraction from fungal endophytes: I. hildebrandtii and I. parasitica seeds were collected from the Plant Genetic Resources Conservation Unit (USDA, ARS). Endophytic material from I. hildebrandtii was obtained by scraping the fungus from the leaf surface approximately two months after seed germination. Sources of fungal DNA from I. parasitica included both ungerminated seeds and leaves collected from two month old plants. To access fungal DNA from I. parasitica, samples were pulverized in a FastPrep 120 (Qbiogene, Irvine, California) at $6 \mathrm{~m} / \mathrm{s}$ for 30 seconds. DNA was obtained from plant material according to the Gene Clean Spin Protocol (Bio 101, Vista, CA).

DNA manipulations: The easA gene from morning glory endophytes was amplified using degenerate eas $A$ primers, the sequences of which were deduced by aligning the eas $A$ genes from Periglandula ipomoeae and Claviceps purpurea. PCR of the easA coding sequence was primed with degenF $\left({ }^{5}\right.$ ATGTCCACRTCSAACCTWTT ${ }^{3}$ ) and degenR $\left({ }^{5}\right.$ TATTCYTTACAGAAGGGATAGTC ${ }^{3}$ ) which yielded a $1.1 \mathrm{~kb}$ fragment. A $25 \mu \mathrm{L}$ reaction contained 1× GoTaq Flexi buffer, $200 \mu \mathrm{M}$ deoxynucleotide triphosphate, 1.5 $\mathrm{mM} \mathrm{MgCl} 2,1 \mu \mathrm{M}$ of each primer, and 2.5 units of Taq DNA polymerase (Promega, Madison, WI, USA). PCR conditions began with an initial denaturing step of $3 \mathrm{~min}$ at $94{ }^{\circ} \mathrm{C}$, followed by 35 cycles of $30 \mathrm{~s}$ at 94 ${ }^{\circ} \mathrm{C}$ followed by $30 \mathrm{~s}$ at $55^{\circ} \mathrm{C}$, and $90 \mathrm{~s}$ at $72{ }^{\circ} \mathrm{C}$, followed by a final extension of $5 \mathrm{~min}$ at $72{ }^{\circ} \mathrm{C}$. PCR products were purified using a QIAquick gel extraction kit (Qiagen, Venlo, Limburg). The easA PCR product was ligated into the TA cloning vector pGEM-T (Promega, Madison, WV, USA). Purified PCR product and plasmid cloned easA product were sequenced at Davis Sequencing (Davis, Calfornia, USA). Generation of transformation construct: The transformation construct was generated by a Splicing by Overlap extension (SOEing) PCR method (Horton, 1995) and contained the endophytic eas A coding sequence under the control of the $A$. fumigatus eas $A$ promoter and attached to the $A$. fumigatus eas $A$ 3'UTR. The $A$. fumigatus easA promoter was amplified using primer promoter-F $\left({ }^{\prime}\right.$ GGTGCGGAGTGCCTACTCTATAG $\left.{ }^{3^{\prime}}\right)$ and overlap primer ovrlapprom-R $\left({ }^{5^{\prime}}\right.$ AGGTTGGACGTGGACATTGCTTCTAATCCACCAAGTACTTG ${ }^{3}$ ) resulting a $0.8 \mathrm{~kb}$ fragment. The eas $A$ coding region was amplified with overlap primers promeasA-F ( $\left({ }^{\prime}\right.$ CAAGTACTTGGTGGATTAGAAGCAATGTCCACGTCCAACCT $\left.{ }^{3}\right)$ and easAutr-R $\left({ }^{\prime}\right.$ CATATTTAATATCCATTCTCGTTGTATTCCTTACAGAAGGGATA ${ }^{3}$ ) producing a $1.1 \mathrm{~kb}$ fragment. The A. fumigatus $3^{\prime}$ UTR region was primed with easAend-F ( ${ }^{5}$ TATCCCTTCTGTAAGGAATACAACGAGAATGGATATTAAATATG $\left.{ }^{3}\right)$ and utr-R ( ${ }^{\prime}$ CAACAGCCCGGGGATACCTTAATTAT ${ }^{3}$ ) yielding a $1 \mathrm{~kb}$ fragment. PCR conditions and protocol are mentioned above.

The promoter and coding region were fused together by SOEing PCR. One $\mu \mathrm{L}$ of purified PCR amplified $A$. fumigatus eas $A$ promoter and one $\mu \mathrm{L}$ of purified $\mathrm{PCR}$ amplified eas $A$ coding region were used as template DNA. The construct was primed with promoter-F ( ${ }^{\prime}$ GGTGCGGAGTGCCTACTCTATAG ${ }^{3}$ ) 
and easAutr-R ( ${ }^{\prime}$ CATATTTAATATCCATTCTCGTTGTATTCCTTACAGAAGGGATA ${ }^{3{ }^{\prime}}$ ) generating a $1.9 \mathrm{~kb}$ fragment. The PCR conditions and protocol are mentioned above with the exception of a two min elongation period.

The complete gene containing both promoter and 3'UTR attached to the easA coding region was primed with promoter-F ( ${ }^{5^{\prime}}$ GGTGCGGAGTGCCTACTCTATAG $\left.{ }^{3^{\prime}}\right)$ and utr-R $\left({ }^{\prime}\right.$ CAACAGCCCGGGGATACCTTAATTAT $^{3^{\prime}}$ ) which yielded a $2.9 \mathrm{~kb}$ fragment. One $\mu \mathrm{L}$ of purified fusion construct and one $\mu \mathrm{L}$ of purified PCR amplified A. fumigatus 3' UTR were used as template DNA. The PCR conditions and protocol are mentioned above with the exception of a three min elongation period.

Fungal transformation and screening: Transformation of an easA ko A. fumigatus culture (Coyle et al., 2010) was performed as described by Ryan et al. (2013). Fungi were co-transformed with easA construct DNA and selectable marker (pAMD1). Transformants were grown on acetamide selective media and screened for the eas A construct by PCR using primers AscrF (5' CCACGTCCAACCTATTCAACGCAC 3') and AscrR (5' CAGAAGGGATAGTCCAGGTA 3') amplifying a 1 kb fragment.

HPLC analysis: Positive transformants were analyzed by HPLC for ergot alkaloid accumulation. Ergot alkaloids were extracted by incubating one $\mathrm{cm}^{2}$ of spore rich fungal matter in $400 \mu \mathrm{L}$ of methanol for 30 minutes, followed by centrifugation for extract clarification. Extracts were then transferred into a C18 column (Prodigy 5- $\mu \mathrm{m}$ ODS3 (150 mm by $4.6 \mathrm{~mm}$ ); Phenomenex, Torrance, CA, USA) and exposed to a binary gradient of solution $\mathrm{A}(5 \%(\mathrm{v} / \mathrm{v})$ acetonitrile plus $95 \%(\mathrm{v} / \mathrm{v})$ aqueous $50 \mathrm{mM}$ ammonium acetate) and solution B ( $75 \%$ acetonitrile plus $25 \%$ aqueous $50 \mathrm{mM}$ aqueous ammonium acetate) as described by Ryan et al. (2013). Samples were compared to authentic cycloclavine standard.

\section{Results and Discussion}

Amplification of easA from the I. hildebrandtii endophyte and its role in ergot alkaloid synthesis.

The eas $A$ coding region from the I. hildebrandtii endophyte was successfully amplified using degenerate primers. Gel electrophoresis revealed the amplification of a single DNA fragment $1.1 \mathrm{~kb}$ in length. The translated endophyte-derived easA sequence was $94 \%$ identical to EasA from $P$. ipomoeae and $85 \%$ identical to the $C$. purpurea-derived EasA (Figure 2), both of which are responsible for agroclavine formation. The easA PCR product contained the eas $A$ start codon but lacked the stop codon as well as an additional $34 \mathrm{bp}$ reads at the $3^{\prime}$ end of the gene due to the annealing position of the reverse degenerate primer.

Involvement of the I. hildebrandtii endophyte-derived easA gene in cycloclavine formation was determined by expressing eas $A$ in an $A$. fumigatus eas $A$ ko strain, which does not synthesize festuclavine 
but rather accumulates precursor chanoclavine-I aldehyde (Coyle et al., 2010). A complete easA gene was constructed by fusing the $A$. fumigatus eas $A$ promoter and $3^{\prime}$-UTR to the endophyte derived eas $A$ coding region by SOEing PCR, which yielded a $2.9 \mathrm{~kb}$ fragment. Since the easA stop codon was not amplified with the coding region, $a^{5^{\prime}} \operatorname{TAA}^{3^{\prime}}$ stop codon was incorporated into the fusion construct.

A. fumigatus easA ko transformants positive for the easA fusion construct were analyzed by HPLC for ergot alkaloid production. Fungal extracts did not contain a metabolite that co-eluted at 52 minutes with cycloclavine standard (Figure 3 ) but rather the metabolites in the transformant co-eluted with festuclavine at 43 minutes and $A$. fumigatus late pathway products: fumigaclavine $A$, fumigaclavine $B$, and fumigaclavine $C$.

Festuclavine is a typical product of $A$. fumigatus EasA activity, and I. hildebrandtii leaves and seeds normally accumulate detectable levels of this compound (Beaulieu et al., 2013) due to expression of a functional eas $A$ gene. In this study, we had hypothesized that a variant eas $A$ allele was responsible for cycloclavine synthesis. Unfortunately, we amplified the typical copy of this gene which is responsible for festuclavine synthesis. Sequencing results of amplified PCR products primed with degenerate oligos gave a single clear sequencing read suggesting no other eas $A$ gene variants were amplified. Future improvements to this study may include using primers with a higher degeneracy value for an increased chance of amplifying variant genes.

Of course, it is plausible that another gene, not related to eas $A$, is responsible for catalyzing the formation of cycloclavine. The I. hildebrandtii endophyte genome has not yet been sequenced, and therefore the presence of uncharacterized functional genes in the ergot alkaloid gene cluster is unknown. DNA sequencing of the cluster from the I. hildebrandtii endophyte may shed light on this theory.

\section{Attempts to amplify easA from the I. parasitica endophyte.}

Unfortunately, the easA coding region from the I. parasitica endophyte was not amplified. A logical explanation for this outcome was the age of $I$. parasitica seeds we obtained. Many of the seeds collected from the Plant Genetic Resources Conservation Unit were over 10 years old. Though seeds can still germinate at this age, the endophyte is not likely to be viable, by analogy with Epichloë species. Lysergol and other ergot alkaloids were not detected in I. parasitica tissues two months after seed germination suggesting the plants were not infected with endophytes. However, the original seeds obtained from USDA ARS were at one time infected with living fungal matter because lysergol was detected in both seeds and two week old plant extracts. Presumably, lysergol was synthesized during seed development where it remained stable until seed germination. Two week old plant extracts 
contained lower concentrations of lysergol compared to seed extracts suggesting the compound was diffusing with tissue growth or possibly degrading. In a continued attempt to amplify the easA coding region, DNA was extracted from ungerminated seeds in hopes that endophyte DNA was still intact; however the easA coding region was not able to be amplified. Young I. parasitica seeds were not obtained due to the fact that this morning glory species is not native to the United States.

A second potential explanation for our inability to amplify the easA coding region may be a lack of primer specificity for the target gene. The ergot alkaloid gene cluster from the $I$. parasitica endophyte has not been sequenced, hence the need for degenerate primers. Amplification of the easA coding region will be more successful upon cluster sequencing.

Future improvements to this study may include obtaining fresh endophyte-infected I. parasitica seeds/plants. DNA extracts from other lysergol-producing fungal species may also be used such as extracts from Ipomoea muricata (Khanuja et al., 2006; Maurya et al., 2012; Patil et al., 2013). It is plausible that another gene, instead of easA, is responsible for lysergine synthesis from chanoclavine-I aldehyde. Comparisons of cluster sequences from lysergol producers may provide insights to this.

\section{References}

Amor-Prats D, Harborne JB. 1993. Allelochemical effects of ergoline alkaloids from Ipomoea parasitica on Heliothis virescens. Birkhäuser Verlag AG 4: 55-61.

Beaulieu WT, Panaccione DG, Hazekamp CS, Mckee MC, Ryan KL, Clay K. 2013. Differential Allocation of seed-borne ergot alkaloids during early ontogeny of morning glories (Convolvulaceae). J. Chem. Ecol. 39: 919-930.

Cheng JZ, Coyle CM, Panaccione DG, O'Connor SE. 2010. Controlling a structural branch point in ergot alkaloid biosynthesis. J. Am. Chem. Soc. 132: 12835-12837.

Coyle CM, Cheng JZ, O'Connor SE, Panaccione DG. 2010. An old yellow enzyme gene controls the branch point between Aspergillus fumigatus and Claviceps purpurea ergot alkaloid pathways. Appl. Environ. Microbiol. 76: 3898-3903.

Horton RM. 1995. PCR-mediated recombination and mutagenesis. Mol. Biotechnol. 3: 93-99.

Khanuja S, Arya JS, Srivastava SK, Shasany AK, Kumar S, Ranganathan T, Darokar MP, Kumar S. 2006. Antibiotic pharmaceutical composition with lysergol as bio-enhancer and method of treatment. United States Patent, Number 20070060604.

Maurya A, Verma RK, Srivastava. 2012. Quantitative determination of bioactive alkaloids lysergol and chanoclavine in Ipomoea muricata by reversed-phase high-performance liquid chromatography. Biomed. Chromatogr. 26: 1096-1100.

Maurya A, Dwivedi GR, Darokar MP, Srivastava SK. 2013. Antibacterial and synergy of clavine alkaloid lysergol and its derivatives against nalidixic acid-resistant Escherichia coli. Chem. Biol. Drug Des. 81: 484-490. 
Panaccione DG, Beaulieu WT, Cook D. 2014. Bioactive alkaloids in vertically transmitted fungal endophytes. Funct. Ecol. 28: 299-314.

Patil S, Nivsarkar M, Anandajiwala S. 2013. Isolation and TLC densitometric quantification of lysergol from the seeds of Ipomoea muricata (Linn.) Jacq. ISRN Chromatography 2013: 1-6.

Ryan KL, Moore C, Panaccione DG. 2013. Partial reconstruction of the ergot alkaloid pathway by heterologous gene expression in Aspergillus nidulans. Toxins 5: 445-455.

Steiner U, Ahimsa-Muller MA, Markert A, Kucht S, Groß J, Kauf N, Kuzma M, Zych M, Lamshoft M, Furmanowa M, Knoop V, Drewke C, Leistner E. 2006. Molecular characterization of a seed transmitted clavicipitaceous fungus occurring on dicotyledonous plants (Convolvulaceae). Planta 224: 533-544.

Steiner U, Leibner S, Schardl CL, Leuchtmann A, Leistner E. 2011. Periglandula, a new fungal genus within the Clavicipitaceae and its association with Convolvulaceae. Mycologia 103: 1133-1145. 


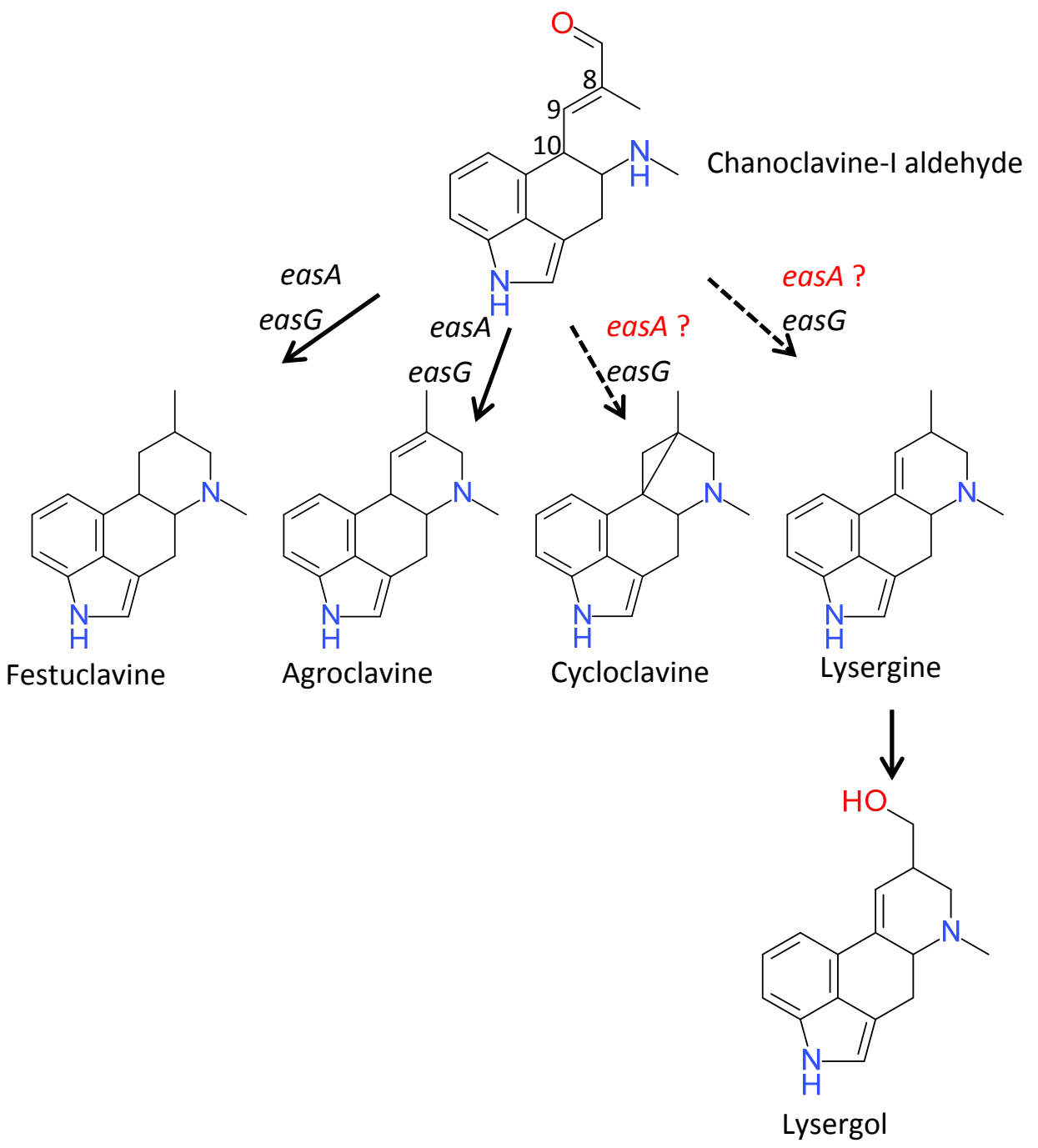

Figure 1: Partial ergot alkaloid pathway. Solid arrows represent known steps in the pathway, while dashed arrows represent hypothesized steps. Genes involved in pathway steps are listed next to arrows. 


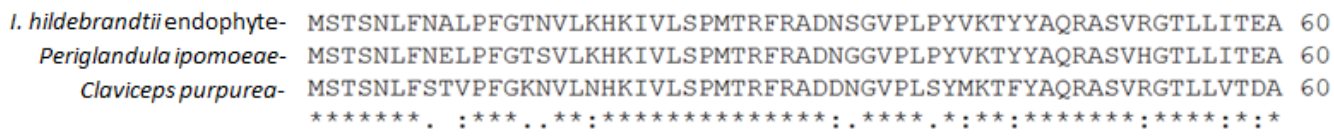

VAICPRAKGEPNVPGIWNDSQIAAWREVVDEVHSKGSVIWLQLWATGRAADTDTLTSNGL 120 VAICPRAKGFPNIPGIWHDSQIAAWKGVVDEVHSKGSVIWLQLWATGRAVDPDTLTSNGL 120 VAICPRTKGEPNVPGIWHKDRIAAWKEVVDEVHSKGSF IWLQLWATGRAADLEALTSRGL 120

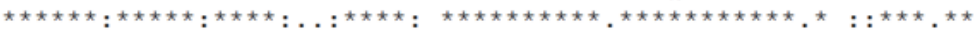

ELESSSEVPVAPGGPTPRPLSEEEIQSYIFDYAQGARNAVHEAGFDGVEIHGANGFLIDQ 180 ELKSSSEVPVAPGEPTPRPLSEEEIQSYIFDYAQGAKNAVHEAGFDGVEIHGANGFLIDQ 180 KLGSSSEVPVAPGEPTPRALDEDEIQQYILDYVQGAKNAVHGAGEDGVEIHGANGFLIDQ 180

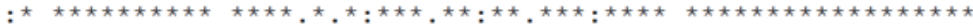

FLQSSCNRRTDQWGGSIENRSRFGLEITRRVIDAVGNDRVGVKLSPWSTFQGMGTMDDLV 240 FLQSSCNRRTDQWGGSIENRSRFGLEITRRVIDAVGNDHVGVKLSPWSTFQGMGTMDDLV 240 FLQSSCNRRTDQWGGSIENRSRFGLEITRGVVDAVGHDRVGMKLSPWSTFQGMGTMDDLV 240

LQFEHFITCLREMDIAYLHLANSRWVEEEDPSIKTHPDVHNETFVRIWGEKRPILLAGGY 300 PQFEHFITRLREMDIAYLHLANSRWVEEEDPSIKTHPDVHNETFVRIWGEKRPILLAGGY 300 PQFEHFITCLREMDIAYLHLANSRWVEEEDPSIRTHPDFHNQTFVQMWGKKRPILLAGGY 300

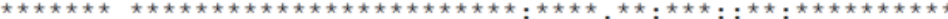

DSDSAQRLVEETYSEQNNVMVVFGRHYISNPDLPFRLKMGIALQGYNRDSFYIPFSGEGY 360 NPDSARRLVDQTYSEQNNVMVVFGRHYISNPDLPFRLKMGIALQRYNRDTFYIPFSGEGY 360 DPDSARRLVDQTYSDRNNVLVVFGRHYISNPDLPFRLRMGIALQKYNRDTFYIPCSGEGY 360

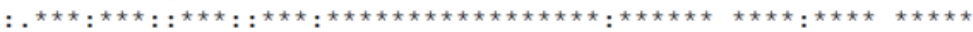

LDYPFCKE----------- 368

LDYPFCKEYLKOGDQETTVA 380

VDYPFCKEYLDQADEAAVAG 380

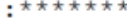

Figure 2: Alignment of easA deduced amino acid sequences from $I$. hildebrandtii fungus with those of $P$.

ipomoeae and C. purpurea. 


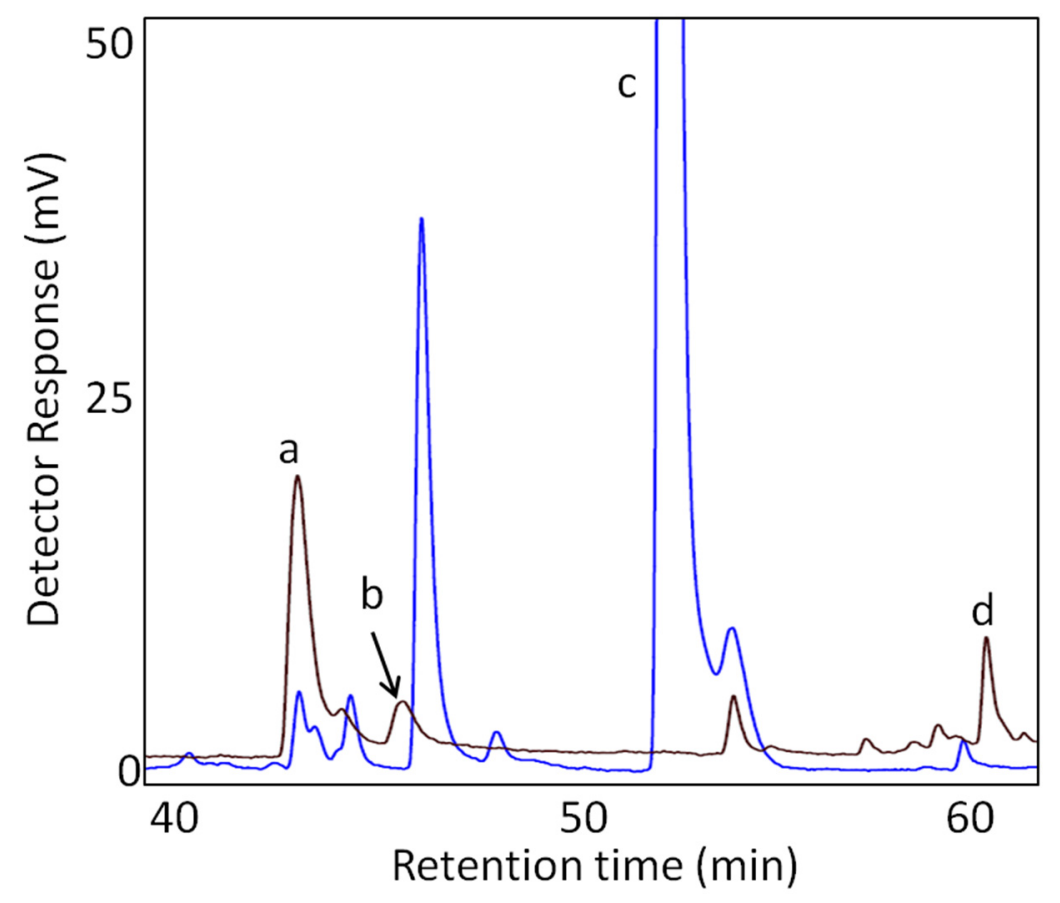

Figure 3: Ergot alkaloid profiles of $A$. fumigatus transformed with endophyte derived eas $A$ construct (black) and known cycloclavine accumulator, Ipomoea hildebrandtii (blue). Fluorescence detector setting at $272 \mathrm{~nm}$ excitation and $372 \mathrm{~nm}$ emissions. $a=$ festuclavine, $b=$ fumigaclavine $A, c=$ cycloclavine, $d=$ fumigaclavine $\mathrm{C}$. 
Chapter 6

Modification of grass endophytes for altered ergot alkaloid production 


\section{Introduction}

Epichloë spp. are endophytes of important forage and turf grasses within the Poaceae. These fungi grow intercellularly throughout above-ground plant tissue and into developing seeds, from which they become transmissible to the next generation of seedlings (Clay and Schardl, 2002). The grass/endophyte symbiosis offers many benefits to the plant host including tolerance to biotic and abiotic stresses (Schardl and Phillips, 1997). Ergot alkaloids produced from endophytes also serve as feeding deterrents to herbivores; though not all endophytes are genetically capable of synthesizing these mycotoxins (Schardl and Phillips, 1997; Schardl et al., 2013). Ergot alkaloid-producing Epichloë spp. generally synthesize lysergic acid amides and ergopeptines.

Ergonovine is a mobile alkaloid possessing both insecticidal and nematicidal properties (Beaulieu et al., 2013; Panaccione, 2010). The only species of Epichloë able to synthesize this bioprotective compound is Epichloë gansuensis harbored in drunken horse grass (Achnatherum inebrians), located in parts of Asia (Schardl et al., 2013). The IpsB and IpsC genes are required for ergonovine synthesis (Figure 1) (Ortel and Keller, 2009). LpsB and LpsC interact to form a peptide synthetase complex that catalyzes the addition of an alanine residue to lysergic acid followed by reduction of the carboxyl group to form ergonovine. The IpsB gene also serves as a branch point in the ergot alkaloid pathway; the encoded enzyme is responsible for both ergonovine (via LpsC/LpsB interaction) and ergopeptine (via LpsA/LpsB interaction) synthesis (Ortel and Keller, 2009; Riederer et al., 1996; Walzel et al., 1997).

Lysergic acid $\alpha$-hydroxyethylamide (LAH) is an ergot alkaloid produced by several species of fungi (Schardl et al., 2013; Castagnoli et al., 1970). The biosynthetic origin of LAH is unknown; though it is plausible that this compound is a derivative of ergonovine due to structural similarity (Figure 1). The eas $P$ and eas $O$ genes are among the few uncharacterized cluster-derived ergot alkaloid synthesizing genes remaining. A correlation exists between the presence of eas $O$ and eas $P$ within the genome of alkaloid producers and LAH accumulation (Schardl et al., 2013). The easO gene encodes a flavin binding monooxygenase which is predicted to oxidize the alpha carbon of the alanine-derived residue of ergonovine. The eas $P$ gene encodes an $\alpha / \beta$ hydrolase fold protein which is believed to hydrolyze its substrate to yield LAH.

The goals of this project were to 1) generate an ergonovine producing strain of Epichloë sp. Lp1 and examine its potential bioprotective capabilities in perennial ryegrass (Lolium perenne), and 2) investigate the roles of eas $\mathrm{O}$ and eas $\mathrm{P}$ in LAH synthesis.

\section{Methods}


Strains and conditions: Epichloë gansuensis var. inebrians E818 was used as a source of genomic DNA. An IpsA knockout strain of Epichloë festucae var. Iolii x E. typhina isolate Lp1 (E. sp. Lp1) was previously generated (Panaccione et al., 2001). E. sp. Lp1 cultures were grown on M102 medium (Bacon et al., 1977 ) at $20^{\circ} \mathrm{C}$. Transformation medium was the same formulation but with $304 \mathrm{~g} / \mathrm{L}$ sucrose. $A$. fumigatus isolate A293 was grown on malt extract medium ( $15 \mathrm{~g} / \mathrm{L}$ malt extract, $20 \mathrm{~g} / \mathrm{L}$ sucrose) at $37^{\circ} \mathrm{C}$, and $A$. nidulans isolate FGSC A767 was grown on modified SYE medium (Ryan et al., 2013). For all fungal transformations, medium was supplemented with $304 \mathrm{~g} / \mathrm{L}$ sucrose.

PCR amplification: The IpsC gene was amplified using primers LpsCF $\left({ }^{\prime}\right.$ TCAGGTGCATCTCAATATGTTCGGC $^{3}$ ) and LpsCR ( ${ }^{\prime}$ CGTCGCAAATAGCAAGGACCTGGATC ${ }^{3}$ ) yielding a 6 kb fragment. A $25 \mu$ L reaction contained $1 \times$ LongAmp Taq reaction buffer, $200 \mu \mathrm{M}$ of each deoxynucleotide triphosphate, $1 \mu \mathrm{M}$ of each primer, and 2.5 units LongAmp Taq DNA polymerase (New England BioLabs, Ipswich, MA, USA). The reaction began with an initial denaturing step of $3 \mathrm{~min}$ at $94{ }^{\circ} \mathrm{C}$, followed by $35 \mathrm{cycles}$ of $30 \mathrm{~s}$ at $94{ }^{\circ} \mathrm{C}$ and $10 \mathrm{~min}$ at $65^{\circ} \mathrm{C}$, with a final extension of $5 \mathrm{~min}$ at $65^{\circ} \mathrm{C}$. To generate transformants containing both IpsC and eas $P$, a single fragment containing both genes was amplified using primers LpsCF $\left({ }^{5}\right.$ TCAGGTGCATCTCAATATGTTCGGC $\left.{ }^{3}\right)$ and easPR $\left(5^{\prime}\right.$ TGCAGCTAAGATGGAATTCGCCAGCG $\left.{ }^{3^{\prime}}\right)$ resulting in an $8 \mathrm{~kb}$ fragment. PCR conditions were the same as above. The eas $O$ gene was amplified using primers easOF $\left(5^{\prime}\right.$ CATGTGAGCAGGGCTGATAGGC ${ }^{3}$ ) and easOR ( ${ }^{5}$ TGCGCCAGAGTTGTAGGATGA ${ }^{3}$ ) which generated a 2.7 $\mathrm{kb}$ fragment. PCR conditions were the same as above with the exception of a 3 minute extension time. SOEing PCR: For eas $P$ and eas $O$ expression in Aspergillus fumigatus, the native promoters of both genes were replaced with the $A$. fumigatus easA promoter using a Splicing overlap extensive (SOEing) PCR method (Horton, 1995). The easA promoter was amplified using primers promF ( ${ }^{5}$ GGTGCGGAGTGCCTACTCTATAG ${ }^{3^{\prime}}$ ) and promR ( ${ }^{\prime}$ TGCTTCTAATCCACCAAGTACTTG ${ }^{{ }^{\prime}}$ ) resulting in a $0.8 \mathrm{~kb}$ fragment. The easP gene was amplified with SOEing overlap primer easpF $\left({ }^{5}\right.$ GTACTTGGTGGATTAGAAGCACCGCTGCTTACAATGGAC ${ }^{3}$ ) and easPR( $\left(5^{\prime}\right.$ TGCAGCTAAGATGGAATTCGCCAGCG $\left.{ }^{3}\right)$ which yielded a $1 \mathrm{~kb}$ fragment. The eas $O$ gene was amplified with SOEing overlap primer ( ${ }^{5}$ 'GTACTTGGTGGATTAGAAGCAACACGATGAATGTCTCAC $^{3}$ ) and easOR ( ${ }^{5^{\prime}}$ TGCGCCAGAGTTGTAGGATGA ${ }^{3^{\prime}}$ ) producing a $2.7 \mathrm{~kb}$ fragment. PCR conditions were the same as listed above except with a 3 minute elongation step. PCR products were gel purified using a QIAquick gel extraction kit (Qiagen, Venlo, Limburg).

For construct fusion, one $\mu \mathrm{L}$ of promoter DNA was mixed with one $\mu \mathrm{L}$ PCR amplified gene product. The complete easP fusion gene was primed with promF ( ${ }^{5}$ GGTGCGGAGTGCCTACTCTATAG ${ }^{3}$ ) and easPR ( ${ }^{5^{\prime}}$ TGCAGCTAAGATGGAATTCGCCAGCG ${ }^{3^{\prime}}$ ) to yielded a $1.8 \mathrm{~kb}$ fragment, and the complete eas $O$ gene fusion was primed with promF $\left({ }^{\prime}\right.$ GGTGCGGAGTGCCTACTCTATAG $\left.{ }^{3}\right)$ and easOR $\left(5^{\prime}\right.$ TGCGCC- 
AGAGTTGTAGGATGA ${ }^{3^{\prime}}$ ) to yield a $3.5 \mathrm{~kb}$ fragment. PCR conditions were the same as listed above except with a 4 minute elongation step.

Transformation and screening: $E$. sp. Lp1 IpsA ko cultures were grown in liquid medium for three days for mycelium growth. A. fumigatus and A. nidulans were grown in malt extract medium overnight at 37 C. All fungi were transformed according to the protocol described by Ryan et al. (2013). E. sp. Lp1 IpsA ko cultures were transformed with either IpsC PCR product or IpsC, eas $P$ and easO PCR products using pBCphleo as a selectable marker. Epichloë transformants were selected on M102 transformation medium with $400 \mu \mathrm{g} / \mathrm{mL}$ phleomycin. Transformants were screened for the presence of each gene using internal primer binding sites. The IpsC gene was screened using primers IpsCscnF $\left(^{5^{\prime}}\right.$ AGCAAGAGCAAGGACCATAC $\left.{ }^{3}\right)$ and IpsCscnR $\left({ }^{5^{\prime}}\right.$ GCTAGAACAGACGATTGCAG $\left.{ }^{3}\right)$ to yield a 990bp fragment. The easP gene was screened using primers easPscnF $\left({ }^{\prime}\right.$ GGATCATCAGAGCCCTTCCA $\left.{ }^{3}\right)$ and easPscnR ( ${ }^{5^{\prime}}$ TGGCATGGCGGTTACTTGGT ${ }^{3}$ ) to yield an 800 bp fragment. The eas $O$ gene was screened using primers easOscnF ( $\left(5^{\prime}\right.$ CGGCACCTATGATCACACATC $\left.{ }^{3}\right)$ and easOscnR $\left(5^{\prime}\right.$ TCACAGCCGTGCTTATCCA $\left.{ }^{3}\right)$ to yield a 500 bp fragment. A $25 \mu \mathrm{L}$ reaction containing 1× GoTaq Flexi buffer, $200 \mu \mathrm{M}$ each deoxynucleotide triphosphate, $1.5 \mathrm{mM} \mathrm{MgCl}_{2}, 1 \mu \mathrm{M}$ of each primer, and 2.5 units of Taq DNA polymerase (Promega, Madison, WI, USA). The reaction began with an initial denaturing step of 3 min at $94{ }^{\circ} \mathrm{C}$, followed by 35 cycles of $30 \mathrm{~s}$ at $94{ }^{\circ} \mathrm{C}, 30 \mathrm{~s}$ at $55^{\circ} \mathrm{C}$, and $1 \mathrm{~min}$ at $72{ }^{\circ} \mathrm{C}$, with a final extension of 5 min at $72{ }^{\circ} \mathrm{C}$. Positive E. sp. Lp1 transformants were sent to Dr. Christopher Schardl (University of Kentucky) for plant infection according the protocol listed by Latch et al. (1985).

Both $A$. fumigatus and $A$. nidulans A767 were transformed with fusion constructs PeasA:eas $P$ and PeasA:easO and selectable marker pCosx (A. fumigatus) or pPyrG (A. nidulans). A. fumigatus transformants were selected on malt extract medium modified with to contain $1 \mathrm{M}$ sucrose and 600 $\mu \mathrm{g} / \mathrm{mL}$ hygromycin, and $A$. nidulans transformants were selected on modified SYE medium (Ryan et al., 2013) lacking both uracil and uridine.

Ergonovine feeding studies: $A$. fumigatus and $A$. nidulans transformants positive for PeasA:eas $P$ and PeasA:eas $O$ were incubated in $200 \mu \mathrm{L}$ malt extract medium supplemented with one $\mu \mathrm{g} / \mathrm{mL}$ ergonovine. $1 \times 10^{6}$ spores were added to the medium and allowed to incubate for five days.

HPLC analysis: Ergot alkaloids were extracted from perennial ryegrass according to the protocol described by Panaccione et al. (2012). Ergot alkaloids were extracted from A. fumigatus and A. nidulans by incubating fungal matter in $200 \mu \mathrm{L}$ of methanol for 30 minutes, followed by centrifugation for extract clarification. Extracts were then transferred into a C18 column (Prodigy 5- $\mu \mathrm{m}$ ODS3 (150 mm by 4.6 $\mathrm{mm})$; Phenomenex, Torrance, CA, USA) and exposed to a binary gradient of solution A $(5 \%(\mathrm{v} / \mathrm{v})$ 
acetonitrile plus $95 \%(\mathrm{v} / \mathrm{v})$ aqueous $50 \mathrm{mM}$ ammonium acetate) and solution $\mathrm{B}$ ( $75 \%$ acetonitrile plus $25 \%$ aqueous $50 \mathrm{mM}$ aqueous ammonium acetate) as described by Ryan et al. (2013). Authentic standards of ergonovine and LAH were used.

\section{Results and Discussion}

\section{Attempts to generate endophytes with altered ergot alkaloid production.}

E. sp. Lp1 IpsA ko was transformed with either IpsC for ergonovine production or IpsC, easO and eas $P$ for ergonovine and LAH production. Two strains transformed with IpsC and six transformants positive for IpsC, easO and eas $P$ were identified and inoculated into perennial ryegrass (Lolium perenne). Ergot alkaloids extracted from grasses were analyzed by HPLC. Unfortunately, all mutant endophyteinfected grass extracts did not contain a metabolite that co-eluted with ergonovine (retention time at 37 minutes) or with LAH standard (isomer retention times at 36 and 38 minutes) (Figure 2). Instead, all grass extracts displayed an ergot alkaloid profile identical to grasses infected with IpsA ko E. sp. Lp1, predominantly synthesizing ergotryptamine.

The formation of ergonovine is dependent on LpsB and LpsC interactions (Ortel and Keller, 2009). The lack of ergonovine production in our transformants may be due to inability of the E. sp. Lp1derived LpsB and E. gansuensis-derived LpsC enzymes to interact in a functional way. LAH is presumably an ergonovine derivative, so the lack of ergonovine would explain the lack of LAH accumulation in endophytes positive for $\operatorname{Ips} C$, eas $O$ and eas $P$. Future modifications to this experiment should include transforming endophytes with both $I p s B$ and $I p s C$ genes amplified from the same organism to ensure enzyme subunit compatibility. An ideal recipient strain for this endeavor is the Epichloë coenophiala mutant generated by Florea et al. (2013), which lacks functional IpsB and IpsC genes.

A second explanation for the absence of ergonovine in our mutant endophytes may be due to a lack of IpsC gene expression. The native IpsC promoter (from E. gansuensis) was not replaced with a functional $E$. sp. Lp1 promoter. Since both fungi are closely related, it was presumed that $E$. sp. Lp1 would recognize E. gansuensis promoters. However, we did not verify expression of the introduced genes with an mRNA analysis and therefore cannot rule out this possibility.

\section{Attempts to determine the involvement of eas $O$ and eas $P$ in LAH synthesis.}

The involvement of eas $\mathrm{O}$ and eas $\mathrm{P}$ in LAH synthesis was additionally tested by transforming both genes into $A$. nidulans and $A$. fumigatus, followed by an ergonovine feeding study. The native promoters of both eas $O$ and easP genes were replaced with the functional $A$. fumigatus eas $A$ promoter by SOEing PCR. A. nidulans is known to recognize the A. fumigatus easA promoter from data obtained in 
the previous chapter. Four $A$. nidulans transformants and three $A$. fumigatus transformants positive for both eas $O$ and eas $P$ constructs were identified. Positive transformants were incubated with one $\mu \mathrm{g} / \mathrm{mL}$ ergonovine for five days followed by an HPLC analysis for LAH accumulation. Unfortunately, fungal extracts from both mutant species did not reveal ergonovine turnover or LAH accumulation.

The lack of LAH synthesis in our ergonovine fed transformants may be due to several reasons. It is possible that ergonovine is not the direct precursor to LAH and cannot be used as a substrate by either EasP or EasO. Lysergyl-alanine is structurally similar to both alkaloids and may serve as an intermediate in LAH synthesis; however, this compound is not commercially available to use in feeding studies. A second explanation for the lack of ergonovine turnover may be that EasP and/or EasO need to interact with the $\mathrm{LpsB} / \mathrm{LpsC}$ peptide synthetase complex to convert ergonovine to LAH. Neither $A$. nidulans or $A$. fumigatus contain IpsB or IpsC genes. A third possibility is that EasP and EasO simply do not contribute to LAH biosynthesis.

Future improvements to this experiment may include introducing eas $O$ and eas $P$ into fungi that naturally express both $I p s B$ and $I p s C$, and therefore form a peptide synthetase complex and synthesize ergonovine. With the exception of mutant strains, few fungal lines are available as recipients for this study. Many natural ergot producers that contain functional $I p s B$ and $I p s C$ genes also contain both eas $P$ and eas $O$ within their genome (Schardl et al., 2013). Claviceps purpurea strain 20.1 is an exception and may serve as a potential gene recipient (Schardl et al., 2013). However, this fungus also expresses IpsA (Figure 1), and therefore contains an active branch point in the ergot pathway making it a less than ideal recipient. An alternative experiment for determining the involvement of eas $O$ and eas $P$ in LAH synthesis is to generate knockout fungal strains. Several fungal lines are available for this experiment such as Epichloë gansuensis, Claviceps paspali, and Claviceps purpurea.

\section{References}

Bacon CW, Porter JK, Robbins JD, Luttrell ES. 1977. Epichloë typhina from toxic tall fescue grasses. Appl. Environ. Microbiol. 34: 576-581.

Bacon CW, White FJ. 1994. Stains, media, and procedures for analyzing endophytes. In: Biotechnology of endophytic fungi of grasses, CRC Press, Tokyo, 47-56.

Beaulieu WT, Panaccione DG, Hazekamp CS, Mckee MC, Ryan KL, Clay K. 2013. Differential Allocation of seed-borne ergot alkaloids during early ontogeny of morning glories (Convolvulaceae). J. Chem. Ecol. 39: 919-930.

Castagnoli N, Corbett K, Chain EB, Thomas R. 1970. Biosynthesis of N-(alpha-hydroxyethyl)lysergamide, a metabolite of Claviceps paspali Stevens \& Hall. J. Biochem. 117: 451-455. 
Clay K, Schardl C. 2002. Evolutionary origins and ecological consequences of endophyte symbiosis with grasses. Amer. Nat. 160: 99-127.

Florea S, Farman ML, Panaccione DG, Yound CA, Schardl CL. 2013. Alteration of the ergot alkaloid profile through chromosome end knockoff. Phytopath. 103: 3340.

Horton RM. 1995. PCR-mediated recombination and mutagenesis. Mol. Biotechnol. 3: 93-99.

Latch G, Christensen M, Gaynor D. 1985. Aphid detection of endophyte infection in tall fescue. New Zeal. J. Agr. Res. 28: 192-132.

Panaccione DG, Johnson RD, Wang J, Young CA, Damrongkool P, Scott B, Schardl CL. 2001. Elimination of ergovaline from a grass-Neotyphodium endophyte symbiosis by genetic modification of the endophyte. Proc. Natl. Acad. Sci. USA 98: 12820-12825.

Panaccione DG. 2010. Ergot alkaloids. The Mycota, Vol. X, Industrial Applications, 2nd Edition (Ed. M. Hofrichter), Berlin-Heidelburg: Springer-Verlag, 195-214.

Panaccione DG, Ryan KL, Schardl CL, Florea S. 2012. Analysis and modification of ergot alkaloid profiles in fungi. Methods Enzymol. 515: 267-290.

Ortel I, Keller U. 2009. Combinatorial assembly of simple and complex D-lysergic acid alkaloid peptide classes in the ergot fungus Claviceps purpurea. J. Biol. Chem. 284: 6650-60.

Riederer B, Han M, Keller U. 1996. D-Lysergyl peptide synthetase from the ergot fungus Claviceps purpurea. J. Biol. Chem. 271: 27524-27530.

Ryan KL, Moore C, Panaccione DG. 2013. Partial reconstruction of the ergot alkaloid pathway by heterologous gene expression in Aspergillus nidulans. Toxins 5: 445-455.

Schardl CL, Phillips TD. 1997. Protective grass endophytes: Where are they from and where are they going? Plant Dis. 81:430-437.

Schardl CL, Young CA, Hesse U, Amyotte SG, Andreeva K, Calie PJ, Fleetwood DJ, Haws DC, Moore N, Oeser B, Panaccione DG, Schweri KK, Voisey CR, Farman ML, Jaromczyk JW, Roe BA, O'Sullivan DM, Scott B, Tudzynski P, An Z, Arnaoudova EG, Bullock CT, Charlton ND, Chen L, Cox M, Dinkins RD, Florea S, Glenn AE, Gordon A, Güldener U, Harris DR, Hollin W, Jaromczyk J, Johnson RD, Khan AK, Leistner E, Leuchtmann A, Li C, Liu JG, Liu J, Liu M, Mace W, Machado C, Nagabhyru P, Pan J, Schmid J, Sugawara K, Steiner U, Takach JE, Tanaka E, Webb JS, Wilson EV, Wiseman $\mathrm{JL}$, Yoshida RY, Zeng Z. 2013. Plant-symbiotic fungi as chemical engineers: multi-genome analysis of the Clavicipitaceae reveals dynamics of alkaloid loci. Plos Genetics 9: e1003323.

Walzel B, Riederer B, Keller U. 1997. Mechanism of alkaloid cyclopeptide synthesis in the ergot fungus Claviceps purpurea. Chem. Biol. 4: 223-230. 


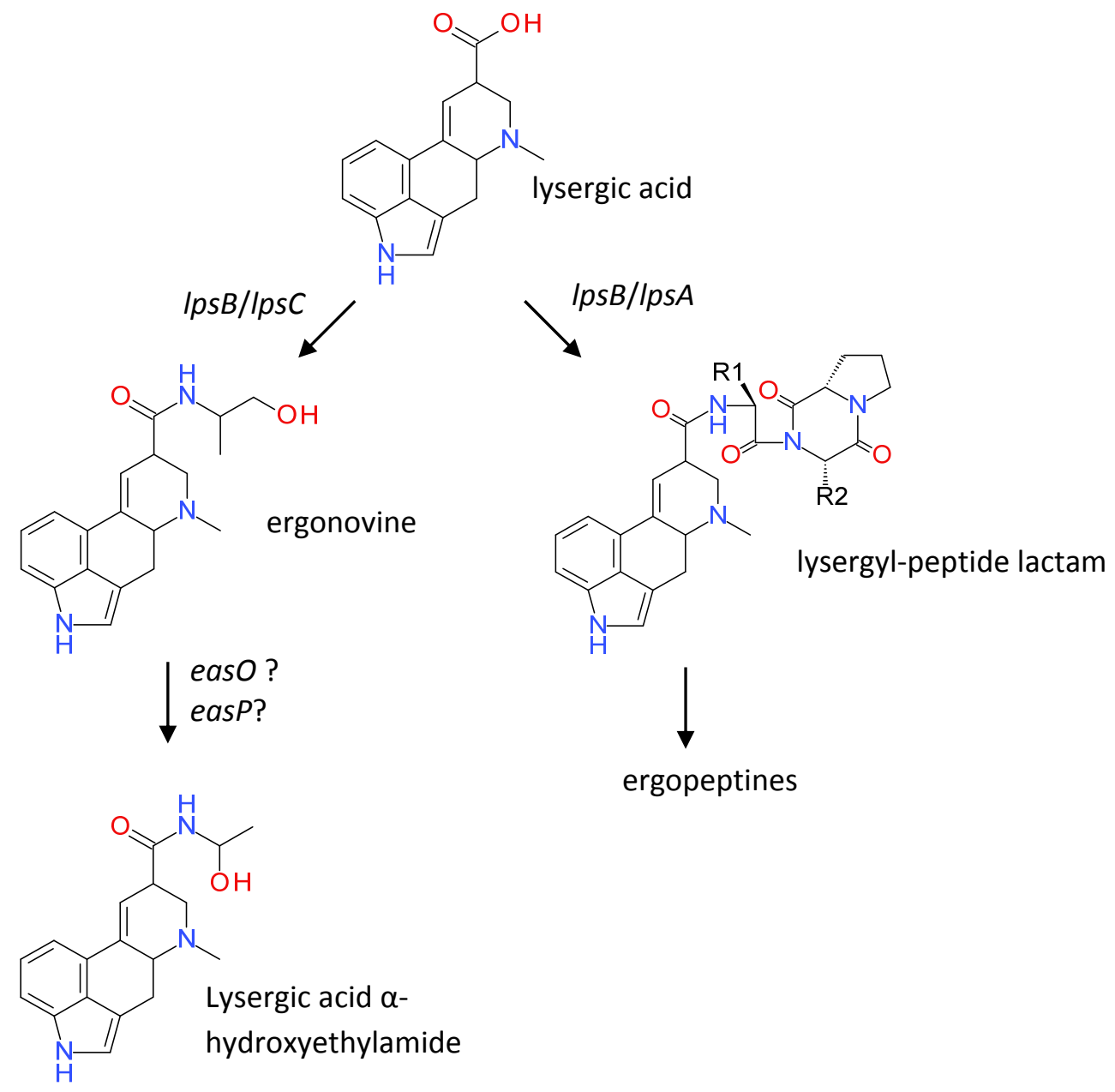

Figure 1: Lysergic acid terminal branch of the ergot alkaloid. Involved genes are shown next to arrows. R groups of lysergyl-peptide lactam represent amino acid residues. 


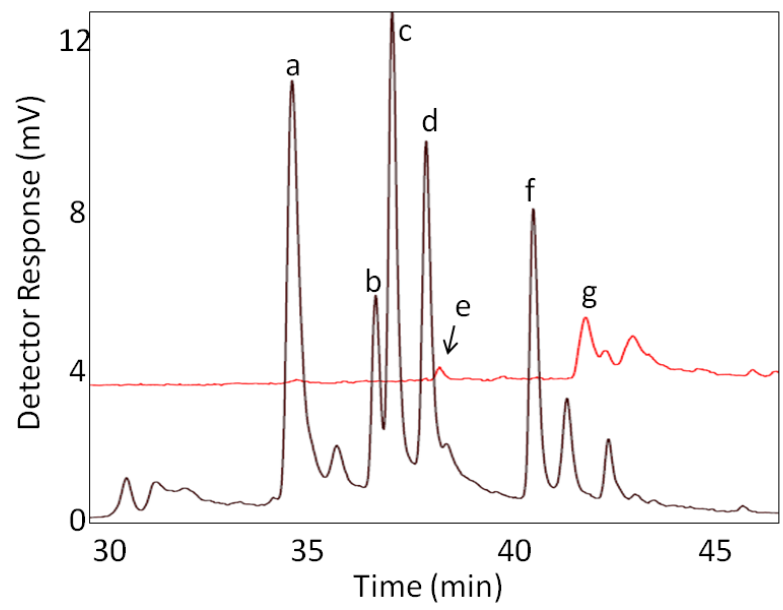

Figure 2: Ergot alkaloid profiles of E. sp. Lp1 transformed with IpsC, eas $P$ and easO (red) and known ergonovine and LAH accumulator, Ipomoea pes-capre (black). Alkaloids were visualized at fluorescence detector setting $310 \mathrm{~nm}$ excitation and $410 \mathrm{~nm}$ emissions. $a=$ ergine, $b=\mathrm{LAH}, \mathrm{c}=$ ergonovine, $d=\mathrm{LAH}$ isomer, $\mathrm{e}=$ oxidized $\mathrm{N}$-Me-DMAT, $\mathrm{f}=$ ergine isomer, $\mathrm{g}=$ ergotryptamine. 


\section{Summary}

Heterologous expression of ergot alkaloid synthesizing (eas) genes in Aspergillus nidulans is an attractive approach to understanding the functions of these genes and their role in the ergot alkaloid pathway. The main focus of this research was to better understand the early steps of alkaloid synthesis by transforming $A$. nidulans with combinations of several known eas genes involved in early pathway steps. A mutant strain of $A$. nidulans expressing $d m a W$, eas $F$, eas $E$, and eas $C$ was generated to determine the sufficiency of these four genes in chanoclavine-I synthesis. Our data revealed the accumulation of chanoclavine-I in mutant extracts suggesting the four genes listed above are sufficient to make A. nidulans synthesize chanoclavine-l. However given our method of study, the involvement of other genes cannot be ruled out.

Interestingly, A. nidulans strain WFC (transformed with dmaW, easF and eas $C$ ) synthesized a unique alkaloid not seen in control strains. The chemical structure of this compound was determined with the use of several chemical techniques including labeling studies, high-resolution mass spectrometry, and NMR. Due do structural similarities with tryptamine, this compound was given the trivial name ergotryptamine. This alkaloid also accumulated in several natural Epichloë isolates, and our investigations revealed that ergotryptamine is the same compound previously described as 6,7secolysergine.

Further investigations of ergotryptamine ruled out its role as a pathway intermediate; however, the accumulation of this compound brought about two new questions: 1) How is EasC involved in alkaloid decarboxylation? and 2) What are the biological activities of ergotryptamine? One key difference between ergotryptamine and precursor N-Me-DMAT is the absence of a functional carboxyl group. The loss of the carboxyl group in this newly identified alkaloid suggests that EasC is involved in alkaloid decarboxylation; however, bioinformatics suggest EasC is a catalase. Further research is needed to verify the role of EasC as a decarboxylase.

The biological activities of ergotryptamine were not investigated due to low quantities produced by fungal strains. Continuing research into the bioactivities of this compound may be advantageous in pharmaceutical and agricultural fields. The research into the early steps of alkaloid synthesis has proven fruitful to the overall understanding of the ergot alkaloid pathway. Valuable data has been derived from this research where it can be applied to numerous scientific fields. 


\section{Katy Ryan}

900 Willowdale Rd, Morgantown WV, 26505

717-836-9057

kryan10@mix.wvu.edu

\section{Education}

Ph.D. in Genetics and Development Biology, Anticipated Fall 2014

West Virginia University, Morgantown WV

Thesis: "Elucidation of the early steps of ergot alkaloid synthesis by pathway reconstruction in Aspergillus nidulans"

Committee Chair: Daniel Panaccione

\section{B.S. in Biology, 2010}

Shippensburg University, Shippensburg PA

Concentration in molecular biology

\section{Research Experience}

Graduate Student Researcher, West Virginia University, 2010-2014

Investigated the biosynthetic origins of ergot alkaloids (mycotoxins) by genetically modifying non-ergot alkaloid producing fungal strains for pathway reconstruction.

- Obtained strong genetic and molecular skills. Ex. DNA isolation, PCR amplification, electrophoresis, bacterial and fungal transformations, DNA cloning, genetic engineering (for generation of fusion proteins and gene knockout systems through homologous recombination), DNA sequencing, bioinformatics.

- Gained expertise in using high performance liquid chromatography (HPLC) and ESI liquid chromatography/ mass spectrometry (LC/MS) to identify, quantify, and isolate fungal metabolites. Utilized isotopes for LC/MS analyses and performed $\mathrm{MS}^{2}$ experiments for chemical structure elucidation.

- Handled and cultured microorganisms.

- Assisted fellow graduate students in identifying fungal isolates using common molecular tools and bioinformatics.

Laboratory Technician, PA Department of Agriculture, 2010

Selected to participate in the Plum Pox Virus Survey and Eradication Program to monitor stone fruit trees for the presence of the plum pox virus.

- Analyzed agricultural samples by enzyme-linked immunosorbent assay (ELISA).

- Participated in field collections and sample preparations.

- Performed basic laboratory maintenance such as restocking solutions and maintaining machinery.

Undergraduate Researcher, Shippensburg University, 2009-2010

Established DNA fingerprints on agricultural isolates of Erwinia tracheiphila.

- Used PCR to amplify enterobacterial DNA markers and established fingerprints based on banding patterns.

- $\quad$ Responsible for basic laboratory maintenance.

\section{Teaching Experience}

Biology Teaching Assistant, West Virginia University, 2012-2014

- Individually taught two Biology 115 (Principles of Biology) laboratories each fall semester.

- Duties included developing and presenting short lectures based on an active learning teaching philosophy, guiding and monitoring students' progress, enforcing laboratory safety, grading homework assignments, proctoring lecture exams, and attending TA laboratory meetings. 
- $\quad$ Served as a TA night supervisor (during $2^{\text {nd }}$ year) to assist new TAs in their duties when the lab manager was not available.

\section{Student Mentor, West Virginia University, 2012-2013}

- Mentored two undergraduate students accepted into the NSF Research Experience for Undergraduates (REU) Program, and oversaw an undergraduate laboratory technician.

- Duties included providing guidance for the development of research projects, teaching basic laboratory skills, and educating on experimental design and result interpretation.

- Assisted students in designing oral and poster presentations.

\section{Publications}

Panaccione DG, Ryan KL, Schardl CL, Florea S. 2012. Analysis and modification of ergot alkaloid profiles in fungi. Methods Enzymol 515; 267-290

Ryan KL, Moore CT, Panaccione DG. 2013. Partial reconstruction of the ergot alkaloid pathway by heterologous gene expression in Aspergillus nidulans. Toxins 5(2); 445-455

Beaulieu WT, Panaccione DG, Hazekamp CS, Mckee MC, Ryan KL, Clay K. 2013. Differential Allocation of seed-borne ergot alkaloids during early ontogeny of morning glories (Convolvulaceae). J Chem Ecol 39(7); 919930

---Manuscript in progress---

Ryan KL, Akhmedov NG, Panaccione DG. 2014. Identification and structural elucidation of a new ergot alkaloid, ergotryptamine, produced by genetically modified Aspergillus nidulans and natural isolates of Epichlö̈ species.

\section{Conferences}

Ryan, KL and Panaccione DG. 2012. Partial reconstruction of the ergot alkaloid pathway in Aspergillus nidulans. Inoculum (suppl. to Mycologia) 63:41

Ryan, KL and Panaccione DG. 2012. Elucidation of the early steps of ergot alkaloid synthesis in Aspergillus nidulans. WVU-Davis College graduate student research competition

Ryan, KL and Panaccione DG. 2013. Analysis of the early steps of the ergot alkaloid pathway by heterologous gene expression in Aspergillus nidulans. Phytopathology 103(Suppl. 4):S4.3

Ryan, KL and Panaccione DG. 2013. Elucidation of the early steps of ergot alkaloid synthesis in Aspergillus nidulans. WVU-Davis College graduate student research competition

Ryan, KL, Akhmendov N, and Panaccione DG. 2014. Reconstruction of the early steps of ergot alkaloid synthesis in Aspergillus nidulans. WVU-Davis College graduate student research competition

\section{Awards and Certifications}

- Davis College graduate student travel award- 2013

- $\quad$ First place Ph.D. presentation award: Davis College graduate student research competition- 2012

- Selected to be a graduate student representative on the WVU Plant and Soil Science director search committee2013

$>$ Underwent laboratory safety training provided by WV Department of Environmental Protection and NIH 2013, 2014

$>$ First Aid and CPR certification provided by American Heart Association-2014 